\title{
A High Resolution View of the Warm Absorber in the Quasar MR 2251-178
}

\author{
J.N. Reeves ${ }^{1,2}$, D. Porquet ${ }^{3}$, V. Braito ${ }^{4}$, J. Gofford ${ }^{1}$, E. Nardini ${ }^{1}$, T. J. Turner ${ }^{2}$, D. M. Crenshaw ${ }^{5}$, S. B. \\ Kraemer 6
}

\begin{abstract}
High resolution X-ray spectroscopy of the warm absorber in the nearby quasar, MR 2251$178(z=0.06398)$ is presented. The observations were carried out in 2011 using the Chandra High Energy Transmission Grating and the XMM-Newton Reflection Grating Spectrometer, with net exposure times of approximately $400 \mathrm{ks}$ each. A multitude of absorption lines from $\mathrm{C}$ to $\mathrm{Fe}$ are detected, revealing at least 3 warm absorbing components ranging in ionization parameter from $\log \left(\xi / \mathrm{erg} \mathrm{cm} \mathrm{s}^{-1}\right)=1-3$ and with outflow velocities $\lesssim 500 \mathrm{~km} \mathrm{~s}^{-1}$. The lowest ionization absorber appears to vary between the Chandra and XMM-Newton observations, which implies a radial distance of between $9-17 \mathrm{pc}$ from the black hole. The soft X-ray warm absorbers likely contribute a negligible $<0.01 \%$ of the bolometric output in terms of their kinetic power. Several broad soft X-ray emission lines are strongly detected, most notably from He-like Oxygen, with FWHM velocity widths of up to $10000 \mathrm{~km} \mathrm{~s}^{-1}$, consistent with an origin from Broad Line Region (BLR) clouds. In addition to the warm absorber, gas partially covering the line of sight to the quasar appears to be present, of typical column density $N_{\mathrm{H}}=10^{23} \mathrm{~cm}^{-2}$. We suggest that the partial covering absorber may arise from the same BLR clouds responsible for the broad soft X-ray emission lines. Finally the presence of a highly ionised outflow in the iron K band from both 2002 and 2011 Chandra HETG observations appears to be confirmed, which has an outflow velocity of $-15600 \pm 2400 \mathrm{~km} \mathrm{~s}^{-1}$. However a partial covering origin of the iron $\mathrm{K}$ band absorption cannot be excluded, resulting from low ionization material with little or no outflow velocity.
\end{abstract}

Subject headings: galaxies:active — quasars: individual: MR 225-178 — X-rays: galaxies

\footnotetext{
${ }^{1}$ Astrophysics Group, School of Physical and Geographical Sciences, Keele University, Keele, Staffordshire, ST5 5BG, UK; j.n.reeves@keele.ac.uk

${ }^{2}$ Center for Space Science and Technology, University of Maryland Baltimore County, 1000 Hilltop Circle, Baltimore, MD 21250, USA

${ }^{3}$ Observatoire Astronomique de Strasbourg, Université de Strasbourg, CNRS, UMR 7550, 11 rue de l'Université, F-67000 Strasbourg, France

${ }^{4}$ INAF - Osservatorio Astronomico di Brera, Via Bianchi 46 I-23807 Merate (LC), Italy

${ }^{5}$ Department of Physics and Astronomy, Georgia State University, Astronomy Offices, One Park Place South SE, Suite 700 , Atlanta, GA 30303, USA

${ }^{6}$ Institute for Astrophysics and Computational Sciences, Department of Physics, The Catholic University of America, Washington, DC 20064, USA
} 


\section{Introduction}

Photo-ionized or "warm" absorbers are commonly observed in at least 50\% of the UV/X-ray spectra of Seyfert 1s and type-1 QSO and are an important constituent of AGN (e.g., Reynolds 1997; Crenshaw et al. 2003; Porquet et al. 2004; Blustin et al. 2005). The Seyfert warm absorbers that are frequently observed at high spectral resolution with XMM-Newton and Chandra are now known to give rise to numerous narrow absorption lines, usually blue-shifted, implying outflowing winds of a few hundred $\mathrm{km} \mathrm{s}^{-1}$ up to a few thousand $\mathrm{km} \mathrm{s}^{-1}$. These arise from various elements over a wide range of ionization parameters, especially from carbon, nitrogen, oxygen, neon, silicon, sulfur, and iron (e.g., Kaastra et al. 2000; Kaspi et al. 2002; Blustin et al. 2002; McKernan et al. 2003).

X-ray spectral signatures of the warm absorber range from the lowly ionized Unresolved Transition Array (UTA) of M-shell iron ( $<$ Fe XVII) at $\sim 16 \AA$ (Sako et al. 2001; Behar et al. 2001) to absorption from highly ionized (H-like and He-like) iron which may originate from an accretion disk wind (e.g., Reeves et al. 2004; Risaliti et al. 2005; Braito et al. 2007; Turner et al. 2008; Tombesi et al. 2010a,b; Gofford et al. 2013). These spectroscopic measurements can reveal crucial information on the outflow kinematics, physical conditions and locations relative to the central continuum source - ranging from the inner nucleus $(0.01 \mathrm{pc})$ to the galactic disk or halo $(10 \mathrm{kpc})$ - which can ultimately unveil the inner structure of quasars (Elvis 2000).

The warm absorption signatures observed in the soft X-ray band cover a wide range of column densities and ionization parameters from $\log \left(N_{\mathrm{H}} / \mathrm{cm}^{-2}\right) \sim 20-23$ and $\log \left(\xi / \mathrm{erg} \mathrm{cm} \mathrm{s}^{-1}\right) \sim-1-31$ These warm absorbers are thought to be typically located on fairly large distances from the central black hole, from their low ionization parameter and velocity values, their (relative) lack of variability, plus in some cases from their inferred low densities (e.g., NGC 3783: Behar et al. 2003; Krongold et al. 2005; Mrk 279: Scott et al. 2004; Ebrero et al. 2010; NGC 4051: Steenbrugge et al. 2009; Mrk 290: Zhang et al. 2011; and Mkn 509: Kaastra et al. 2012). These soft X-ray warm absorbers can be associated with, for example, a wind originating from the putative parsec scale torus (Blustin et al. 2005) or the latter stages of an accretion disc wind which has propagated out to larger radii (Proga \& Kallman 2004; Tombesi et al. 2013). By virtue of their low outflow velocities the soft X-ray warm absorbers are thought to only have a weak feedback effect in their host galaxy. Indeed, the mechanical power imparted by individual warm absorption components is very low, typically $\lesssim 0.01 \%$ of an AGN's bolometric luminosity ( $\left.L_{\text {bol }}\right)$ (e.g., Blustin et al. 2005), which is significantly lower than the $\sim 0.5 \%$ of $L_{\text {bol }}$ thought necessary for feedback to affect the host galaxy (Hopkins \& Elvis 2010). However, Crenshaw \& Kraemer (2012) have recently shown that this $\sim 0.5 \%$ threshold can be exceeded provided the mechanical power is integrated over all UV and X-ray absorption components, at least in the case of a few moderate-luminosity local AGN.

Recent systematic archival XMM-Newton and Suzaku studies have shown that Fe XXV-XXVI absorption lines are present in the X-ray spectra of $\gtrsim 40 \%$ of radio-quiet AGN in the local universe with $z<0.1$ (Tombesi et al. 2010a, 2011, 2012; Patrick et al. 2012; Gofford et al. 2013) and also in a sample of 30 local

\footnotetext{
${ }^{1}$ The ionization parameter is defined as $\xi=L_{\mathrm{ion}} / n R^{2}$ (Tarter et al. 1969), where $L_{\mathrm{ion}}$ is the $1-1000$ Rydberg ionizing luminosity, $n$ is the electron density and $R$ is the distance of the ionising source from the absorbing clouds.
} 
Broad Line Radio Galaxies (Tombesi et al. 2013, in preparation) which thus suggests that they may represent an important addition to the commonly held AGN unification model (e.g., Antonucci 1993; Urry \& Padovani 1995). In comparison to the soft-band absorbers these hard X-ray absorbers generally have much more extreme parameters, with $\log \left(N_{\mathrm{H}} / \mathrm{cm}^{-2}\right) \approx 23-24$ and $\log \left(\xi / \mathrm{erg} \mathrm{cm} \mathrm{s}^{-1}\right) \approx 3-6$, and their outflow velocities relative to the host galaxy can reach mildly relativistic values. The large inferred velocities combined with the short time-scale variability sometimes exhibited by the absorption features - point to an origin more likely associated with a wind which is launched from the surface of the accretion disc itself (e.g., Pounds et al. 2003; Reeves et al. 2009; Gofford et al. 2011; Tombesi et al. 2012). In this scenario the inferred mass outflow rates for disc-winds are often comparable to those of the matter which accretes onto the central black hole and the consequent mechanical power can also be a sizeable fraction (i.e., $\geq$ few percent) of an AGN's bolometric luminosity (e.g., Chartas et al. 2002; Pounds et al. 2003; Gibson et al. 2005; Reeves et al. 2009; Gofford et al. 2011; Tombesi et al. 2012).

MR 2251-178 ( $z=0.06398$; Bergeron et al. 1983; Canizares et al. 1978) is one of the X-ray brightest AGN in the local universe $\left(L_{2-10 \mathrm{keV}} \sim 2-9 \times 10^{44} \mathrm{erg} \mathrm{s}^{-1}\right)$. It was the first quasar identified through X-ray observations (Cooke et al. 1978; Ricker et al. 1978) and the first AGN known to host a warm absorber (Halpern 1984). The quasar is located on the outskirts of a cluster of $\sim 50$ galaxies (Phillips 1980) and is surrounded by an extended nebula of diffuse gas out to $10-20 \mathrm{kpc}$, which gives rise to [O II], [O III] and $\mathrm{H} \alpha$ emission at optical wavelengths (Macchetto et al. 1990; Phillips 1980). The source has a central black hole mass of $\sim 2.4 \times 10^{8} \mathrm{M}_{\odot}$ (Dunn et al. 2008), is observed to be a weak radio emitter (with a radio loudness parameter, $R_{\mathrm{L}}=F_{5 \mathrm{GHz}} / F_{4400 \AA}=-0.43$; Reeves \& Turner 2000), and has a Fanaroff-Riley type I (FR I) radio morphology (Macchetto et al. 1990).

The first detailed study of MR 2251-178 in the X-ray regime was conducted by Halpern (1984) who, using spectra from the Einstein X-ray observatory, noticed soft X-ray variability on time-scales of $\sim 1 \mathrm{yr}$ caused by changes in both the column density of photoionized material along the line of sight and an associated change in the materials ionization state. The ionization state of the absorbing material was also later found to be strongly correlated with the source luminosity, with the absorber appearing to become more ionized when the source was at a larger luminosity, which thus strongly suggested the presence of partiallyionized 'warm' material along the line of sight (Mineo \& Stewart 1993). Subsequent observations with EXOSAT, Ginga and BeppoSAX found the broad-band X-ray spectrum could be well described by a powerlaw of photon-index $\Gamma \sim 1.6-1.7$ which is absorbed by a column density of around a few $\times 10^{22} \mathrm{~cm}^{-2}$ (Pan et al. 1990; Mineo \& Stewart 1993), and a high-energy roll-over at around $100 \mathrm{keV}$ (Orr et al. 2001). In the UV, Monier et al. (2001) found absorption lines due to Ly $\alpha, \mathrm{N}$ V and C IV with a systematic blueshift of $\sim 300 \mathrm{~km} \mathrm{~s}^{-1}$; the C IV absorption in particular showed variability over a period of roughly 4 years which constrained the absorption clouds to within $r \lesssim 2.4 \mathrm{kpc}$ of the continuum source (Ganguly et al. 2001).

Kaspi et al. (2004) performed a detailed spectral and temporal study of MR 2251-178 using a series of ASCA, FUSE, BeppoSAX and XMM-Newton observations which spanned a period of $\sim 8.5 \mathrm{yrs.} \mathrm{In} \mathrm{con-}$ firmation of previous studies Kaspi et al. (2004) also found the continuum to be described by an absorbed power-law of photon index $\Gamma \sim 1.6$, but also found that the continuum required a supplementary softexcess at $E<2 \mathrm{keV}$ to achieve an acceptable fit to the soft X-ray data. The grating spectrum from the 
$X M M-N e w t o n / R G S$ revealed the warm absorber in MR 2251-178 to be multi-phase, consisting of at least two or three ionised absorption components with column densities in the range $10^{20-22} \mathrm{~cm}^{-2}$, all of which had physical properties which appeared to vary between observations in accord to what was reported by Halpern (1984). This led Kaspi et al. (2004) to propose a scenario where absorption clouds were moving across the line of sight over a time-scale of 'several months'. In the FUSE spectrum further UV absorption lines from C III, H I and O VI were detected with velocity shifts similar to those found by Monier et al. (2001). A 2002 Chandra/HETG observation of MR 2251-178 was published by Gibson et al. (2005). There the authors found evidence for a highly-ionised Fe XXVI Ly $\alpha$ absorption line with a substantial blueshifted velocity, $v_{\text {out }}=-12700 \pm 2400 \mathrm{~km} \mathrm{~s}^{-1}$. By considering the kinematics of the absorber Gibson et al. (2005) inferred that unless the absorber is of a low global covering fraction (in terms of the total fraction of $4 \pi \mathrm{sr}$ covered by the absorber) the mass-loss rate in MR 2251-178 is at least an order of magnitude larger than the source accretion rate.

A recent analysis over the $0.6-180.0 \mathrm{keV}$ broad-band X-ray spectrum has been performed by Gofford et al. (2011) combining a Suzaku observation of MR 2251-178 performed in May 2009 and Swift/BAT data as part of the 58-months all-sky-survey (Baumgartner et al. 2010). In accordance with previous observations, the authors found that the general continuum can be well described by a power-law with $\Gamma=1.6$, an apparent soft-excess below $1 \mathrm{keV}$ and considerable curvature above around $\sim 10 \mathrm{keV}$. However, the authors found that a good fit can also be found with a softer $\Gamma \sim 2.0$ power-law absorbed by a column of $N_{\mathrm{H}} \sim 10^{23} \mathrm{~cm}^{-2}$ which covers $\sim 30 \%$ of the source flux. This softer photon index value is more consistent with that found generally in radio-quiet quasars (e.g., Reeves \& Turner 2000; Porquet et al. 2004; Piconcelli et al. 2005; Scott et al. 2011). In addition, numerous significant warm absorption lines were detected (at the $>99 \%$ confidence level from Monte Carlo simulations) and associated to Fe UTA, Fe L shell (blend of $2 s \rightarrow 3 p$ transitions from Fe XXIII-XXIV), S XV, S XVI and Fe XXV-XXVI lines. Gofford et al. (2011) found at least 5 ionised absorption components with $10^{20} \lesssim N_{\mathrm{H}} \lesssim 10^{23} \mathrm{~cm}^{-2}$ and $0 \lesssim \log \xi / \mathrm{erg} \mathrm{cm} \mathrm{s}{ }^{-1} \lesssim 4$ are required to achieve an adequate spectral fit of all these absorption features.

In this paper the analysis of an unprecedented deep follow-up campaign of MR 2251-178 in 2011 with XMM-Newton and Chandra, is presented. The XMM-Newton and Chandra observations were both performed as a large observing program, with the observations within about a month of each other. The exposure times of these observations, of $\sim 400 \mathrm{ks}$, is significantly greater than obtained in the previous 2002 Chandra/HETG and XMM-Newton observations (net exposures of $\sim 140$ ks and $60 \mathrm{ks}$ respectively). The increased exposure times make it possible to study the warm absorber in this quasar in unprecedented detail and resolution, with the RGS and HETG gratings on-board XMM-Newton and Chandra respectively. Thus the overall goal of this campaign was to obtain high signal-to-noise and high resolution spectroscopy of MR 2251-178 in order to measure the properties of the primary continuum emission and in particular the ionized absorption and outflow along the line of sight.

This paper is organized as follows. In section 2 , we describe the data reduction of both RGS and HETG observations. Section 3 is devoted to the initial spectral fitting of the HETG data, to atomic line detections and identifications as well to the initial kinematics of the absorption lines. Section 4 presents photoionization 
modeling of the X-ray absorption in the RGS and HETG spectra combining fully and partial covering warm absorber components; in addition the variability of the X-ray absorption components and the possible presence of a highly ionized absorber are examined. Section 5 focuses on the modeling of the emission line spectrum, especially the O VII line complex. In Section 6, we discuss about the origins and infer some physical properties of the absorption and emissions media observed in MR 2251-178 and compare them to those found in other AGN.

Values of $H_{0}=70 \mathrm{~km} \mathrm{~s}^{-1} \mathrm{Mpc}^{-1}$, and $\Omega_{\Lambda_{0}}=0.73$ are assumed throughout and errors are quoted at $90 \%$ confidence $\left(\Delta \chi^{2}=2.7\right)$, for 1 parameter of interest. All spectral parameters are quoted in the rest-frame of the quasar, at $z=0.06398$ (Bergeron et al. 1983), unless otherwise stated.

\section{Observations and Data Reduction}

\subsection{XMM-Newton Observations of MR 2251-178}

XMM-Newton observed MR 2251-178 three times from 11-17 November 2011, over 3 consecutive satellite orbits. Each observation was approximately $130 \mathrm{ks}$ in length, with the details of the 3 observations listed in Table 1. First order dispersed spectra were obtained with the Reflection Grating Spectrometer (den Herder et al. 2001) and were reduced using the RGSPROC script as part of the XMM-Newton SAS software v11.0. The spectra from each of the orbits were found to be consistent with each other, with the only variation being due to a $10 \%$ change in the count rate of the source over the 3 observations. Therefore spectra and response files for each RGS were combined to give a single spectrum with a total net exposure of $389.1 \mathrm{ks}$. There were no periods of strong background flares during the observations, the background rate in each RGS being only 7-8\% of the total source rate. Prior to spectra analysis, channels due to bad pixels on the RGS CCDs were ignored as well as the two malfunctioning CCDs for RGS 1 and RGS 2 respectively.

The net background subtracted count rates were $0.496 \pm 0.001 \mathrm{~s}^{-1}, 0.535 \pm 0.001 \mathrm{~s}^{-1}$ for RGS 1 and RGS 2 respectively, yielding a total of over $4 \times 10^{5}$ counts for the two RGS spectra together. Spectra were binned into $\Delta \lambda=0.02 \AA$ bins, which over-samples the RGS spectral resolution by a factor of $\times 4$ compared to the FWHM resolution. Due to the high count rate statistics, $\chi^{2}$ minimization was employed in the subsequent spectral fitting. An additional $\pm 3 \%$ systematic error was added in quadrature to each combined RGS spectrum, in order to allow for systematic differences between the two grating spectra. A constant multiplicative offset was subsequently allowed between the RGS 1 and RGS 2 in all the spectral fits, which was found to be within $\pm 3 \%$. Data were fitted over the $0.33-2.0 \mathrm{keV}$ energy range in the observed frame.

\subsection{Chandra HETG Observations of MR 2251-178}

The High Energy Transmission Grating (HETG) onboard Chandra (Weisskopf et al.2000; Canizares et al. 2005) also observed MR 2251-178 from 26 September to 2 October 2011, occurring approximately 40 days 
before the XMM-Newton observations. As per the XMM-Newton observations, the Chandra observations occurred over 3 consecutive orbits, with the last sequence somewhat shorter than the first two - see Table 1 for details. Spectra were extracted with the CIAO package v4.3. Only the first order dispersed spectra were considered for both the MEG (Medium Energy Grating) and HEG (High Energy Grating) and the \pm 1 orders for each grating were subsequently combined for each sequence. No significant spectral variability was observed between the 3 sequences and the spectra were consistent, with only modest $\sim 10 \%$ variations in source flux. Therefore the spectra were combined from all three sequences to yield a single 1st order spectrum for each of the MEG and HEG, yielding respective net source count rates of $0.485 \pm 0.001 \mathrm{~s}^{-1}$ and $0.245 \pm 0.001 \mathrm{~s}^{-1}$ respectively for a total exposure time of $392.9 \mathrm{ks}$. Thus the total counts obtained exceeded $1.9 \times 10^{5}$ and $9.5 \times 10^{4}$ counts for MEG and HEG respectively. Note that the background contribution towards the count rate was negligible.

The resultant 2011 source spectra were subsequently binned to $\Delta \lambda=0.02 \AA$ and $\Delta \lambda=0.01 \AA$ bins for MEG and HEG respectively, which samples their respective FWHM spectral resolutions. The MEG and HEG spectra were analyzed over the energy ranges of $0.5-5.0 \mathrm{keV}$ and $1.0-9.0 \mathrm{keV}$ respectively. The C-statistic was employed in the subsequent spectral fits to the HETG, as although the overall count rate is high, towards the lower energy (longer wavelength) end of each grating spectrum the total source counts per bin drops below $N<20$ in some bins. In the case of $\chi^{2}$ minimization, this would lead to the continuum level being somewhat underestimated at soft X-ray energies.

An archived Chandra HETG observation of MR 2251-178 also took place 11 September 2002, with a total net exposure of $146.3 \mathrm{ks}$. First order spectra for MEG and HEG were re-extracted as above, yielding count rates of $0.317 \pm 0.001 \mathrm{~s}^{-1}$ and $0.164 \pm 0.001 \mathrm{~s}^{-1}$ respectively. Thus the 2011 observation was approximately $50 \%$ higher in count rate or flux than the earlier 2002 observation and therefore the 2002 dataset provides a lower flux comparison spectrum. The data were binned and analyzed over the same energy ranges as per the 2011 observation and the C-statistic was employed in all subsequent spectral fits.

\section{Initial Spectral Fitting}

\subsection{The Overall Spectral Form}

Initially we concentrated on the 2011 RGS and HETG observations. All parameters are given in the rest frame of the quasar at $z=0.06398$, unless otherwise stated and spectral parameters are quoted in energy units (thus $1 \mathrm{keV}$ is equivalent to $12.3984 \AA$ ). In all the fits, a Galactic absorption of hydrogen column density of $N_{\mathrm{H}}=2.4 \times 10^{20} \mathrm{~cm}^{-2}$ (Kalberla et al. 2005) was adopted, modeled with the "Tuebingen-Boulder" absorption model (TBABS in XSPEC) using the cross-sections and abundances of Wilms et al. (2000). Figure1 shows the overall 2011 fluxed RGS spectrum of MR 2251-178, plotted against a powerlaw of $\Gamma=2$ in the soft X-ray band and in the quasar rest frame at $z=0.06398$. The spectrum shows several clear signatures of a warm absorber and emitter. A deep absorption trough is present between $0.7-0.8 \mathrm{keV}$ which is most likely identified with an unresolved transition array (UTA), due to $2 p \rightarrow 3 d$ transitions from lower ionization Mshell iron (i.e. Fe less ionized than Fe XVII) (Behar et al. 2001). The iron M-shell UTA has been commonly 
observed in high resolution grating spectra of many AGN (McKernan et al. 2007), e.g., IRAS 13349+2438: (Sako et al. 2001), NGC 3783: (Kaspi et al. 2000, 2001; Krongold et al. 2003), NGC 5548: (Kaastra et al. 2002; Andrade-Velázquez et al. 2010), Mrk 509: (Pounds et al. 2001; Yaqoob et al. 2003; Smith et al. 2007), NGC 7469: (Blustin et al. 2007), Mrk 841: (Longinotti et al. 2010), IC 4239A: (Steenbrugge et al. 2005b), NGC 3516: (Holczer \& Behar 2012), Ark 564: (Papadakis et al. 2007), MCG-6-30-15: (Lee et al. 2001; Turner et al. 2004), NGC 4051: (Pounds et al. 2004a), Mrk 279: (Costantini et al.2007), I Zw1: (Gallo et al. 2004), 1H 0419-577: (Pounds et al. 2004b), PG 1114+445: (Ashton et al. 2004).

Several narrow absorption lines appear to be present between $0.85-1.0 \mathrm{keV}$, likely due to $\mathrm{K}$-shell $1 s \rightarrow 2 p$ lines of Neon as well as higher ionization L-shell $(2 p \rightarrow 3 d)$ lines of iron (i.e. Fe XVII-XXII). A broad absorption trough appears to be present near $1.3 \mathrm{keV}$ in the rest frame, close to the expected $\mathrm{K}$ shell lines of $\mathrm{Mg}$, the origin of which is discussed in Section 4. Strong and resolved line emission is also especially prominent in the RGS 1 spectrum between $0.56-0.58 \mathrm{keV}$, at the expected energy of the O VII triplet.

For comparison, the fluxed 2011 HETG spectrum of MR 2251-178 is shown in Figure2, The spectrum plotted is against a power-law of photon index $\Gamma=1.6$ for comparison purposes only; as is discussed later in Section 4.2, the likely intrinsic photon index of the source is perhaps much steeper $(\Gamma \gtrsim 2)$ once all the layers of absorption in MR 2251-178 are accounted for. Although the power-law provides a good representation of the HETG spectrum above $3 \mathrm{keV}$, the data/model ratio residuals show pronounced curvature due to the presence of the known warm absorber in this AGN. Indeed fitting a single power-law (modified by Galactic absorption only) provided a very poor representation of the whole HETG spectrum fitted from $0.5-9.0 \mathrm{keV}$, with a very hard photon index of $\Gamma=1.33 \pm 0.02$ and an unacceptable fit statistic of $C=3806.4$ for 2360 degrees of freedom (dof). Note a multiplicative cross-normalization constant was included between the MEG and HEG spectra, the HEG normalization was found to be slightly lower $(0.97 \pm 0.01)$ than the MEG (which was normalized to 1.00).

In order to investigate and identify the atomic lines present in the HETG spectra, a more complex continuum shape was adopted in order to better account for the clear spectral curvature. A power-law continuum was adopted, modified by a neutral partial covering absorber (the PCFABS model in XSPEC). While this simple partial coverer is not meant to provide a physical description of the spectrum, its advantages are that it provides a better parameterization of the spectral curvature, while not imparting any discrete atomic lines on the spectrum, thus providing a reference continuum from which lines can be identified against. A similar approach was also taken to provide an initial parameterization of the broad-band Suzaku spectrum of MR 2251-178 (Gofford et al. 2011). In addition to the partial covering absorption, a phenomenological absorption edge component was initially included to account for the pronounced spectral drop above $0.7 \mathrm{keV}$, due to a possible combination of the Fe M-shell UTA and O VII edge. Again this was not meant to provide a physical fit to the spectrum. The edge energy was $E=730.6 \pm 2.1 \mathrm{eV}$ with an optical depth of $\tau=0.36 \pm 0.05$. The partial coverer had a column density $N_{\mathrm{H}}=(2.9 \pm 0.2) \times 10^{22} \mathrm{~cm}^{-2}$ and a covering fraction of $0.35 \pm 0.03$, while the photon index was $\Gamma=1.69 \pm 0.03$. The overall fit statistic was much improved compared to the power-law only case, with $C=3047$ for 2356 degrees of freedom. 


\subsection{Atomic Lines in HETG Spectrum}

Figure 3(MEG) and Figure 4(HEG) show the residuals against the neutral partial covering model in the soft X-ray band below $2 \mathrm{keV}$. A wealth of absorption lines are clearly present in the HETG spectrum against the continuum model over the $0.7-2.0 \mathrm{keV}$ energy range (or 6-18 $\AA$ ). In order to parameterize the lines, successive narrow Gaussian absorption lines were included in the continuum model; an individual line was deemed to be statistically significant if its addition to the model resulted in an improvement of the fit statistic of $\Delta C>9.2$, corresponding to $99 \%$ significance for 2 interesting parameters. The width of the absorption lines was initially assumed to be less than the instrumental resolution. The parameters of all 31 of the statistically significant absorption lines detected in the soft X-ray HETG spectrum are shown in Table 2. A narrow structure is also clearly present in the UTA region around $0.73-0.76 \mathrm{keV}$, these are parameterized

by 2 lines in Table 2, which by comparison with the blends of transitions noted in Behar et al. (2001) may be due to iron in the ionization states Fe VII-X.

Further low ionization gas appears to be present in the form of a multitude of inner K-shell lines of $\mathrm{Ne}, \mathrm{Mg}$ and $\mathrm{Si}$. These are $1 s \rightarrow 2 p$ absorption lines whereby the L-shell is partially occupied, i.e. due to charge states corresponding to $\mathrm{Li}, \mathrm{Be}, \mathrm{B}, \mathrm{C}, \mathrm{N}$, O-like etc ions. We refer to Behar \& Netzer (2002) for a compilation of these inner shell lines, adopting the known energies (wavelengths) of these lines from this paper in Table 2. Indeed such lines have been detected in other high signal to noise grating spectra of Seyfert 1 AGN, such as in NGC 3783 (Kaspi et al. 2002; Blustin et al. 2002), NGC 4151 (Kraemer et al. 2005), Mrk 509 (Kaastra et al. 2011a), NGC 3516 (Holczer \& Behar 2012), NGC 4051 (Lobban et al. 2011) and NGC 5548 (Steenbrugge et al. 2005a). In the MR 2251-178 HETG spectrum, absorption lines due to Ne V-VIII (i.e. C-like through to Li-like ions) are detected from $0.87-0.91 \mathrm{keV}(13.6-14.3 \AA$ ) in the rest frame (Figure 3). Similarly $1 s \rightarrow 2 p$ inner shell lines from $\mathrm{Mg}$ are detected due to Mg VI-IX (N-like through to Be-like ions), from $1.26-1.33 \mathrm{keV}(9.3-9.8 \AA)$. Likewise inner shell absorption is also detected from $\mathrm{Si}$, from $\mathrm{Si}$ VIII-XI (N-like to Be-like) around $1.8 \mathrm{keV}(6.5-7.0 \AA)$. The inner shell absorption is also independently detected in the HEG (Figure 4) as well as the MEG (Figure 3) spectra. Thus the detection of the strong Fe M-shell UTA, plus the inner-shell absorption due to $\mathrm{Ne}, \mathrm{Mg}$ and $\mathrm{Si}$ suggests the imprint of a significant amount of absorption due to both low and high ionization gas in MR 2251-178.

Absorption lines due to more highly ionized gas are also significantly detected in the HETG spectrum. $\mathrm{He}$ and $\mathrm{H}$-like lines of $\mathrm{O}, \mathrm{Ne}, \mathrm{Mg}$ and $\mathrm{Si}$ are all detected (with the exception of the $\mathrm{O}$ VII $1 s \rightarrow 2 p$ line due to the lack of $\mathrm{S} / \mathrm{N}$ below $0.6 \mathrm{keV}$ in the MEG spectrum). In some cases, higher order $1 s \rightarrow n p$ lines are detected, especially in the case of Ne IX where the series of resonance lines up to $1 s \rightarrow 6 p$ are seen. Higher ionization L-shell lines of iron are also present, e.g. from Fe XIX-XXII. The spectra over the $\mathrm{S}$ and Fe K band are also shown Figure 4, although note that neither strong emission nor absorption features appear to be present in these parts of the spectrum. In the $\mathrm{S}$ band, weak absorption may be present at the expected energies of the He and Li-like lines of $\mathrm{S}$, although they are below the formal detection threshold. The details of the iron $\mathrm{K}$ band spectrum will be discussed further in Section 4.4.

It is also apparent from Table 2 that most of the measured rest frame energies of the absorption lines are close to the known atomic energies. This suggests that the outflow velocity of the soft X-ray absorbing gas 
is relatively small. We discuss below some of the velocity profiles of the strongest detected $\mathrm{H}$ and He-like lines.

\subsection{Atomic Lines in RGS Spectrum}

The RGS provides an energy coverage of $0.3-2.0 \mathrm{keV}$ with high throughput and therefore provides a high quality view of the soft X-ray warm absorber, with a lower energy bandpass than Chandra HETG. The initial analysis of the absorption line spectrum suggests that multiple absorption components may be required in order to model the wide range in the ionization state of the gas, e.g. covering for instance Fe VII-XXII, Ne V-X or Mg VI-XII.

Indeed enlarged portions of the RGS spectrum of MR 2251-178 are shown in Figures 5 and 6 Note these are plotted in the observed frame and not the rest frame. The warm absorber is clearly complex, comprising a wealth of atomic features. Notably, inner-shell (Li-like and below) and higher-order (i.e., the $1 s \rightarrow n p$ transitions, where $n \geq 3$ ) absorption lines are detected throughout the spectrum, due to $\mathrm{C}, \mathrm{N}$, $\mathrm{O}, \mathrm{Ne}$ and $\mathrm{Mg}$. Figure 5 shows that the higher-order line series of $\mathrm{C} \mathrm{VI}$ is particularly prominent, while $\mathrm{N}$ VI, N VII, O VII and O VIII also have higher-order line series, with each ion reaching at least the $1 s \rightarrow 4 p$ transition.

Complementing the array of absorption lines there is also some interesting interplay between emission and absorption components; e.g. see the O VII line at $517-539 \mathrm{eV}(23-24 \AA)$ observed frame in Figure 5 The $\mathrm{O}$ VII $\left(1 s 2 p \rightarrow 1 s^{2}\right)$ emission line complex is superimposed on by three narrow absorption lines corresponding to inner-shell absorption due to O V (line 11, Figure 5) and the two lines which make up the O VI $\left(1 s^{2} 2 s \rightarrow 1 s 2 s 2 p\right)$ doublet (lines 13, 14, Figure 5). Again, similar structures are present at other energies, with N VII, O VIII and Ne IX all showing emission superposed by absorption. The nature of the emission line spectrum will be discussed further in Section 5.

From panels (a) and (b) of Figure 6, both Neon and Magnesium also show evidence for inner-shell absorption from at least their Be-like ionisation states (Behar \& Netzer 2002). Indeed, the inner-shell lines for $\mathrm{Mg}$ in particular occur throughout the $\sim 1.2-1.3 \mathrm{keV}$ energy range, as per the HETG. This appears to be the origin of the absorption trough visible in Figure 1 and first noted in the lower resolution Suzaku spectrum of MR 2251-178 published by Gofford et al. (2011). The complete list of atomic lines identified in the RGS data - including details such as the responsible ion, the electron transition and the centroid energies in the source rest-frame - is given in Table 3 .

\subsection{Velocity Profiles}

We constructed velocity profiles of the strongest $\mathrm{H}$ and He-like absorption lines identified in the above HETG and RGS spectra. In each case the profiles were constructed by taking the ratio of the data to the best fit parameterization of the continuum model described above and transposing them into velocity space 
around the known lab frame energy (wavelength) of each line. For the H-like ions, the C VI, N VII, O vIII, $\mathrm{Ne}$ X, Mg XII and Si XIV profiles have been produced, with the profiles plotted in Figure7 Note that the $\mathrm{C}$ VI line corresponds to the $1 s-3 p$ absorption line (as the $1 s-2 p$ line at the redshift of MR 2251-178 is close to the edge of the RGS bandpass), while the other profiles correspond to the $1 s-2 p$ lines. Similarily, profiles were also constructed for the He-like resonance lines of N VI, O VII, Ne IX, Mg XI and Si XIII and are shown in Figure 8 (note only the first 4 profiles are actually plotted here). In the case of the He-like ions, the $1 s-3 p$ lines of $\mathrm{O}$ VII and Ne IX, are used instead of the $1 s-2 p$ lines, due to contamination with other lines present in the spectrum. Overall the profiles from the $\mathrm{C}, \mathrm{N}$ and $\mathrm{O}$ lines were derived from the RGS data in the soft X-ray part of the spectrum (taking the mean of RGS 1 and 2 where both were available), while the $\mathrm{Ne}, \mathrm{Mg}$ and Si profiles were derived from the HEG data at higher energies. Note that negative velocities indicate blue-shift throughout this papen 2 . The profiles are as measured from the data, without correcting for the spectral resolution of the instrument.

The subsequent lines were fitted with Gaussian profiles and the results of the fits are shown in Table 4 which gives both the overall velocity shift $\left(v_{\text {out }}\right)$ of the line profile (as determined from the centroid of the Gaussian profile) as well as the observed $1 \sigma$ velocity width of the profile $\left(\sigma_{\text {obs }}\right)$. Firstly it can be seen both from the profiles themselves and the fits that the outflow velocities of the lines tend to decrease in magnitude with increasing ionization state, e.g. from $\mathrm{C}$ through to Si. For instance for the H-like ions, C VI and N VII profiles have a velocity shift of $v_{\text {out }} \sim-450 \mathrm{~km} \mathrm{~s}^{-1}$, with the Mg XII profile having a formal upper limit on the outflow velocity of only $v_{\text {out }}<40 \mathrm{~km} \mathrm{~s}^{-1}$, while the velocity centroids for O VIII and Ne X are somewhat intermediate in value. We note that a similar possible trend was found in emission in the Seyfert 2 NGC 1068 (Kinkhabwala et al. 2002), whereby the higher energy (excitation) lines had somewhat lower velocities.

The velocity profiles and fits also indicate that a second higher velocity component may be present in the lower energy lines of C VI, N VI and N VII, with an outflow velocity of $v_{\text {out }} \sim-2000 \mathrm{~km} \mathrm{~s}^{-1}$. Such a component is not present in the higher energy lines. The outflow velocities of the possible higher velocity components are also given in Table 4 noting that the line width of this component was assumed to be the same as for the respective lower outflow velocity lines. Thus while we note the possible presence of a higher velocity component to some of the lines, we do not discuss this further here, as the improvement in fit statistic upon adding this second velocity component (see Table 4) was generally less than the more robust low velocity component which is always present.

The observed velocity widths of the Gaussian profiles $\left(\sigma_{\text {obs }}\right)$ are also given in Table 4 These are not corrected for instrument resolution, however for comparison the $\sigma$ widths of the RGS (RGS 1+2 combined) varies between $\sigma=300-380 \mathrm{~km} \mathrm{~s}^{-1}$ for C VI to O VIII and for the HEG between $\sigma=120-230 \mathrm{~km} \mathrm{~s}^{-1}$ for $\mathrm{Ne} X$ to Si XIV. The intrinsic line widths corrected for instrument resolution $\left(\sigma_{\text {int }}\right)$ are also given in Table 4 Thus some of the line profiles appear resolved, with typical widths of $\sigma_{\text {int }}=300-400 \mathrm{~km} \mathrm{~s}^{-1}$, while the higher energy lines (e.g. Mg XII and Si XIII) appear to be unresolved, similar to the possible above trend in outflow velocity.

\footnotetext{
${ }^{2}$ Note that any upper limits on outflow velocities are expressed as absolute values for clarity.
} 


\section{Photoionization Modeling of the X-ray Absorption Spectrum}

Given the substantial presence of partially ionized gas in the X-ray spectrum of MR 2251-178, we attempted to model the absorption spectrum with photoionized grids of models using the XSTAR code v2.2 Kallman et al. 2004). Absorption grids were generated in the form of XSPEC multiplicative tables (or mtables). The absorption spectra within each grid were computed between $0.1-20 \mathrm{keV}$ with $N=10000$ spectral bins. The photoionizing X-ray continuum between 1-1000 Rydberg was assumed to be a power-law of a photon index $\Gamma=2$, except for the grid which covered the lowest range in ionization, which we discuss further below. Given the narrow (or unresolved) widths of the absorption lines detected in the Chandra HETG, grid turbulence velocities of either $\sigma=100 \mathrm{~km} \mathrm{~s}^{-1}$ or $\sigma=300 \mathrm{~km} \mathrm{~s}^{-1}$ were generated; grids with higher turbulences all gave substantially worse fits in the models considered below. An electron density of $n_{\mathrm{e}}=10^{10} \mathrm{~cm}^{-3}$ was assumed for the absorption grids, although we note that the absorption spectra are largely insensitive to the density over a wide range of values. Solar abundances were adopted for all the abundant elements, using the values of Grevesse \& Sauval (1998), except for Ni which is set to zero (the default option within XSTAR).

We generated one generic grid of models that covered a wide range in ionization and column density parameter space, from $N_{\mathrm{H}}=1 \times 10^{18} \mathrm{~cm}^{-2}$ to $N_{\mathrm{H}}=3 \times 10^{24} \mathrm{~cm}^{-2}$ and $\log \left(\xi / \mathrm{erg} \mathrm{cm} \mathrm{s}^{-1}\right)=0-5$ in logarithmic steps of $\Delta\left(\log N_{\mathrm{H}}\right)=0.5$ and $\Delta(\log \xi)=0.5$ respectively. A turbulence velocity of $\sigma=$ $100 \mathrm{~km} \mathrm{~s}^{-1}$ was used. This grid was used to fit the high ionization absorption components, as well as the possible partial covering absorption which we discuss further below. A separate more finely tuned grid (covering a narrower range of parameters) was generated with the specific purpose of modeling the low ionization absorption in the MR 2251-178 spectrum, especially the Fe M-shell UTA and the inner-shell lines. The column density of this low ionization grid covered the range from $N_{\mathrm{H}}=0.5-5.0 \times 10^{21} \mathrm{~cm}^{-2}$ in steps of $\Delta N_{\mathrm{H}}=1 \times 10^{20} \mathrm{~cm}^{-2}$, with the ionization range extending from $\log \left(\xi / \mathrm{erg} \mathrm{cm} \mathrm{s}^{-1}\right)=0-3$ in 15 steps of $\Delta(\log \xi)=0.2$. A fine spectral resolution of $N=10^{5}$ points over an energy range of $0.1-20 \mathrm{keV}$ was also employed. A turbulence velocity of $\sigma=100 \mathrm{~km} \mathrm{~s}^{-1}$ was also adopted. The other significant difference with this absorption grid was that a steeper photoionizing X-ray continuum of $\Gamma=2.5$ was employed, the requirement for this is discussed further in Section 4.2.

\subsection{XMM-Newton RGS}

We first considered the RGS spectrum. The initial analysis of the absorption line spectrum from the HETG and RGS observations in Section 3 suggests that multiple absorption components may be required in order to model the wide range in the ionization state of the gas, e.g. covering for instance Fe VII-XXII, $\mathrm{Ne}$ V-X or Mg VI-XII.

In order to model the absorption spectrum we successively added individual components of absorbing gas, fully covering the line of sight to the source, until the fit statistic was no longer improved at the $99.9 \%$ confidence level. Three components of fully covering gas are formally required in the RGS model, which are listed as components 1-3 in Table 5. The lowest ionization absorber (component 1) was modelled by the 
low ionization XSTAR grid as described above and components 2-3 by the higher ionization grid. We note that the continuum itself was assumed to be a power-law of variable photon index, absorbed by the Galactic column, while we no longer retain either the ad-hoc absorption edge or the simple neutral partial coverer in the models. However we do allow for at least one additional component of partially ionized absorbing gas (as modeled by an XSTAR grid) to partially cover the X-ray source, in addition to the three fully covering components of gas described above, which appears to be required statistically to achieve a good fit. Soft $\mathrm{X}$-ray emission lines are also added to the model as Gaussians when statistically required by the data at $>99 \%$ and will be discussed in detail later. Thus the phenomenological form of the spectral model fitted to the RGS data is:-

$$
F(E)=\text { tbabs } \times \text { comp } 1 \times \operatorname{comp} 2 \times \operatorname{comp} 3 \times\left[\text { pow }_{\text {uncov }}+\text { Gauss }+\left(\mathrm{pc}_{1} \times \text { pow }_{\text {cov }}\right)\right]
$$

where here comp 1-3 represent the 3 fully covering warm absorber components, Gauss represents the Gaussian emission lines and tbabs the Galactic absorption. The partial covering absorber is represented by $\mathrm{pc}_{1}$ which covers a fraction $f_{\text {cov }}$ of the line-of-sight to the $\mathrm{X}$-ray source, while $1-f_{\text {cov }}$ is subsequently unattenuated by the partial covering component. Thus the fraction of the continuum that is absorbed simply given by the respective ratio of the power-law normalizations, i.e:- $f=N_{\text {cov }} /\left(N_{\text {cov }}+N_{\text {uncov }}\right)$. The spectral parameters of the RGS fit are listed in Table 5

Overall the three warm absorber components that are required to model the RGS spectrum cover the range in column from $N_{\mathrm{H}}=1.5-3.6 \times 10^{21} \mathrm{~cm}^{-2}$ and ionization parameter from $\log \left(\xi / \mathrm{erg} \mathrm{cm} \mathrm{s}{ }^{-1}\right)=$ $1.27-2.80$. Consistent outflow velocities are found for the low and medium ionization components 1 and 2, with $v_{\text {out }}=-480 \pm 40 \mathrm{~km} \mathrm{~s}^{-1}$ and $v_{\text {out }}=-460 \pm 60 \mathrm{~km} \mathrm{~s}^{-1}$ respectively. However the highest ionization component 3 does not require an outflow velocity (formally consistent with zero) and only a limit can be placed with $v_{\text {out }}<130 \mathrm{~km} \mathrm{~s}^{-1}$. We note that the lack of any outflow velocity of component 3 also appears consistent with the velocity profile analysis in Section 3.4, where the velocities of the higher excitation lines appear to be lower. The column density $\left(6 \times 10^{22} \mathrm{~cm}^{-2}\right)$ and ionization $\left(\log \left(\xi / \mathrm{erg} \mathrm{cm} \mathrm{s}^{-1}\right)=1\right)$ of the partial covering component are not well constrained in the RGS fit, mainly because of the limited higher energy bandpass of the RGS makes it difficult to constrain multiple continuum components, while the partial coverer itself does not impart discrete detectable lines upon the soft X-ray spectrum (but it does impart continuum curvature). Thus its column and ionization have been fixed in the model, while we note that these values are consistent with those obtained with the HETG in Section 4.2.

Nonetheless the partial coverer is certainly required in the model, the fit statistic is increased by $\Delta \chi^{2}=$ 192.4 upon removing the partial coverer from the model and refitting; its exclusion leads to systematic broad residuals in the data/model ratio suggesting the continuum is inadequately modeled. The covering fraction of the partial coverer is $f_{\text {cov }}=0.61 \pm 0.05$. Overall the fit statistic for the best-fit warm absorber model is $\chi^{2} /$ dof $=2991.7 / 2562$, while the continuum photon index upon modeling all the three required components of warm absorption is steeper, with $\Gamma=2.32 \pm 0.08$. The warm absorber model reproduces well the absorption lines observed in the RGS spectrum, as shown by the solid line in Figures 5 and 6 . We also note that addition to the warm absorption, an additional neutral component of absorption is required in 
the rest frame of MR 2251-178. However its column density is quite small, $N_{\mathrm{H}}=(2.8 \pm 0.3) \times 10^{20} \mathrm{~cm}^{-2}$, and it may plausibly be associated with absorption in the quasar host galaxy rather than the AGN.

The relatively low turbulence velocity (of $\sigma=100 \mathrm{~km} \mathrm{~s}^{-1}$ ) of the warm absorber components aides in the modeling of the higher order lines, as some of the $1 s \rightarrow 2 p$ lines may lie on the saturated part of the curve of growth. This means the some of the higher order lines can be of comparable strength as the $1 s \rightarrow 2 p$ lines, while some of the line series are detected up to $1 s \rightarrow 6 p$. Indeed the warm absorber model matches well the profiles of the higher order lines, as can be seen in Figures 5 and 6 .

To correctly account for the intensity of the low ionisation lines the absorbing grid requires a much softer (steeper) input continuum than the other higher ionization absorption components (which have $\Gamma_{\text {input }}=$ 2.0 ), in order not to over-ionize the gas and reduce their depth in the model. The necessary power-law continuum required by the XSTAR grid in order to model the low ionization lines is $\Gamma_{\text {input }}=2.5$. This is much softer than what has typically been found for MR 2251-178 assuming a fully-covering absorption model, which is of the order of $\Gamma=1.6-1.7$ (Pan et al. 1990; Mineo \& Stewart 1993; Kaspi et al. 2004; Gibson et al. 2005). However the underlying soft X-ray photon index recovered in the RGS spectrum ( $\Gamma=2.32 \pm 0.08$ ), after the required absorbing layers of gas are accounted for, is in reasonable agreement with the required photon index to reproduce the soft X-ray lines. This lends weight to the notion that MR 2251-178 may, indeed, have an intrinsically soft continuum which is partially-covered by a complex and stratified absorber. We discuss this further in Section 4.2.

\subsection{Chandra HETG}

The above best-fit model was then applied to the 2011 HETG spectrum, allowing the continuum and warm absorber parameters to vary between the datasets. A second partial covering component of higher column density of $\sim 7 \times 10^{23} \mathrm{~cm}^{-2}$ was added to the model, as the direct application of the RGS model gave a slight excess at higher energies in the HETG spectrum. Otherwise the model construction applied to the HETG data is identical to the RGS.

The absorber fit parameters applied to the 2011 HETG spectrum are also listed in Table 5 The parameters of the 3 warm absorber components are rather similar to those obtained from the RGS data, with most of the values consistent within the errors between the observations. Similar to the RGS, the warm absorber column densities cover the narrow range $N_{\mathrm{H}}=1.5-2.1 \times 10^{21} \mathrm{~cm}^{-2}$, while the ionization spans a range from $\log \left(\xi / \mathrm{erg} \mathrm{cm} \mathrm{s}^{-1}\right)=1.15-2.9$. There is evidence for a small change in the ionization of the warm absorber of the low ionization component 1 , increasing from $\log \left(\xi / \mathrm{erg} \mathrm{cm} \mathrm{s}^{-1}\right)=1.15 \pm 0.05$ to $\log \left(\xi / \mathrm{erg} \mathrm{cm} \mathrm{s}^{-1}\right)=1.27 \pm 0.02$ between the HETG and RGS, following the same direction as the 0.4-2.0 keV continuum flux which also increased from the HETG to the RGS, we discuss this further in Section 4.3 below. The column density of component 1 is consistent between observations, with $N_{\mathrm{H}}=$ $2 \times 10^{21} \mathrm{~cm}^{-2}$, although the outflow velocity is slightly smallet 3 , with $v_{\text {out }}=-315 \pm 40 \mathrm{~km} \mathrm{~s}^{-1}$. The

\footnotetext{
${ }^{3}$ The differences are likely within the absolute wavelength scales of the HETG and RGS.
} 
ionization and columns of components 2 and 3 are consistent within the errors, while as per the RGS, the highest ionization component 3 does not require any outflow, as noted above.

Figure 9 shows the relative contributions of each of the 3 warm absorbers components against a powerlaw continuum. The lowest ionization component 1 (top panel) contributes the lower ionization ions, i.e. O V-VII, Ne V-VIII, Mg VI-IX, Si VIII-XI as well as M-shell iron, as expected. The higher ionization components produce most of the He and $\mathrm{H}$-like ions, as well as the higher ionization (L-shell) iron ions (see lower panels).

\subsubsection{The Nature of the Photoionizing Continuum}

The HETG has a wider bandpass and higher resolution than the RGS, which enables some additional tests to be applied to the inner-shell lines in particular. Figure 10 shows a comparison between the fit to the warm absorber when the low ionization component (component 1 ) of XSTAR absorption has a $\Gamma_{\text {input }}=2.5$ input photoionizing continuum (blue line) or $\Gamma_{\text {input }}=2.0$ (red line). For the case of the harder $\Gamma=2$ input continuum, the model is clearly unable to account for the depth of the inner-shell (Li-like and below) charge states of $\mathrm{Ne}$ or $\mathrm{Mg}$, whereas the $\Gamma_{\text {input }}=2.5$ absorber is able to model the low ionization absorption lines. This suggests that the softer input continuum is strongly required to model the absorption. The absorption grid with the steeper continuum also provides a better fit to the Fe M-shell UTA and also the Silicon innershell lines. These differences are reflected in the fit statistic, which for the $\Gamma=2$ grid is $C=2665.9$ for 2335 degrees of freedom, whereas for the $\Gamma=2.5$ grid the fit statistic is $C=2542.4$ for the same number of degrees of freedom, corresponding to a difference of $\Delta C=123.5$.

Overall the photon index of the continuum recovered after modeling all the layers of absorption is $\Gamma=2.13 \pm 0.10$. Thus the index is somewhat flatter than in the RGS $(\Gamma=2.32)$, but this may reflect the fact that the RGS is more sensitive at soft X-ray energies than the HETG, especially if the intrinsic continuum has subtle curvature, becoming slightly steeper towards lower energies. Note that Figure 2 also shows the level of the intrinsic continuum (the dashed blue line) after correcting for all the absorbing layers of gas. Thus the observed continuum without modeling the absorption (which would otherwise appear to have a very hard photon index of $\Gamma=1.3$ ) does not necessarily represent the intrinsic emission, where

$\Gamma z 2$, more typical of radio-quiet quasars (e.g., Reeves \& Turner 2000; Porquet et al. 2004; Scott et al. 2011).

The partial covering components also appear to be required by the data. The moderate column partial covering component (named pc 1, Table 5) appears well constrained, with $N_{\mathrm{H}}=5.5 \pm 0.3 \times 10^{22} \mathrm{~cm}^{-2}$ and $\log \left(\xi / \mathrm{erg} \mathrm{cm} \mathrm{s}^{-1}\right)=1.04_{-0.11}^{+0.08}$, while its covering fraction is $f_{\text {cov }}=0.4 \pm 0.1$. The highest column component ( $\mathrm{pc} 2$, Table 5) is less well constrained, but the fit is still worse by $\Delta C=31.6$ if this component is removed from the model and the continuum refitted. The removal of the pc 2 absorber results in the fitted photon index hardening from $\Gamma=2.13 \pm 0.10$ to $\Gamma=1.77 \pm 0.05$. Furthermore if the more moderate column partial coverer (pc1) is also removed then the fit is considerably worse $\Delta C=213.1$ and the photon index then becomes an unphysical $\Gamma=1.49 \pm 0.03$. 
Such a hard continuum slope also poses a problem for the modeling of the warm absorber components, as the low ionization (inner shell) absorption requires a soft input photoionizing continuum of $\Gamma \sim 2.5$ as above, which cannot be recovered in the model without applying the partial covering absorption. The other possibility is that the intrinsic continuum shape and high energy SED are unusual, consisting of a rather hard powerlaw component $\Gamma \sim 1.5$ (and much harder than usually observed in radio-quiet quasars), then softening to an index of $\Gamma \gtrsim 2.5$ at soft X-ray energies. The broad band continuum modeling will be explored in more detail in a forthcoming paper (Nardini et al. 2013, in prep), where the XMM-Newton EPIC and Optical Monitor data will be considered, as well as archival Suzaku and Swift/BAT observations, thereby covering the optical/UV through to hard X-ray bandpass.

We note that although a softer continuum does provide a better fit to the inner shell lines and some improvement to the Fe UTA, the model fits for these inner shell features is dependent on the calculation of the ionization balance for these elements. For example, in their analysis of the 900ksec HETG spectrum of NGC 3783, Netzer et al. (2003) noted that their best warm absorber model did not accurately reproduce the Fe UTA due to the predicted iron being too highly ionized. Netzer et al. (2003) suggested that the problem was the lack of accurate low-temperature $(\Delta \mathrm{n}=0)$ dielectronic recombination (DR) rates for the M-shell sequence of iron (Fe IX - Fe XVI). Following this, Netzer (2004) and Kraemer et al. (2004) incorporated estimated $\Delta \mathrm{n}=0$ DR rates into the codes ION (Netzer 1996) and Cloudy (Ferland et al. 1998), respectively, and demonstrated that such rates would shift the overall ionization balance of M-shell iron downward, hence solving the problem described by Netzer et al. (2003).

More recently, DR rates have been computed (Badnell 2006) for the M-shell states of iron, which are included within XSTAR. These are an order of magnitude greater than the radiative recombination rates for these ions and several times greater than the estimated DR rates from Netzer (2004) and Kraemer et al. (2004). Furthermore, these rates have been confirmed in storage-ring experiments (Schmidt et al. 2006). However while for the same physical parameters as those used in Netzer et al. (2003), Cloudy models using the new DR rates predict similar $\mathrm{C}, \mathrm{N}$, and $\mathrm{O}$ column densities, the predicted Fe ionization is now too low to fit the UTA. Although it may be possible to recover the fit by changing model parameters (e.g., the continuum slope), these results may also indicate that some process which mitigates the effects of the new DR rates is not being accurately treated. One possibility is (multi-electron) autoionization following innershell ionization (D. Savin, private communication). In any event, given such sensitivity to the accuracy and availability of atomic data, the exact parameterization of the low ionization absorber could differ, with the ionization perhaps somewhat lower than currently inferred by XSTAR.

\subsection{Variability of the $X$-ray Absorption}

The best fit absorption model to the 2011 HETG spectrum was also applied to the earlier 2002 HETG observation. The signal to noise of the 2002 observation is substantially lower, due to the overall lower flux level (and count rate) and shorter exposure of this observation (see Table 1), which means that most of the individual absorption lines were not detected (see Gibson et al. 2005 for a description of this dataset). 
However the same spectral model can still be applied to the 2002 data, allowing the continuum and warm absorber parameters to vary between the observations. For ease of comparison the photon index of the 2002 observation was tied to that of the 2011 observation, i.e. $\Gamma=2.13$. The column and ionization of the partial covering components were also fixed to the 2011 values, as otherwise they are less well determined, although the covering fractions were allowed to vary. The warm absorber parameters (column, ionization, outflow velocity) were allowed to vary between the observations.

The absorber parameters of the 2002 observation are shown in Table 5 Again the absorption values are largely consistent between the 2002 and 2011 HETG observations, as well as with the 2011 RGS observations, suggesting that the absorber components appear stable over time. The main parameter that does appear to change is the ionization of the low ionization component 1 absorber. Indeed if the 2011 RGS observation is also considered, the ionization of component 1 appears to increase from $\xi=8.1_{-2.5}^{+3.5} \mathrm{erg} \mathrm{cm} \mathrm{s}^{-1}$ (Sept 2002/HETG) to $\xi=14.1 \pm 1.6 \mathrm{erg} \mathrm{cm} \mathrm{s}^{-1}$ (Sept 2011/HETG) to $\xi=18.6 \pm 0.8 \mathrm{erg} \mathrm{cm} \mathrm{s}^{-1}$ (Nov 2011/RGS). Indeed the changes in $\xi$ appear increase in direct proportion to the observed $0.5-2.0 \mathrm{keV}$ band flux, varying from $0.75 \pm 0.01 \times 10^{-11} \mathrm{erg} \mathrm{cm}^{-2} \mathrm{~s}^{-1}$ (Sept 2002/HETG) to $1.33 \pm 0.01 \times 10^{-11} \mathrm{erg} \mathrm{cm}^{-2} \mathrm{~s}^{-1}$ (Sept 2011/HETG) to $1.80 \pm 0.01 \times 10^{-11} \mathrm{erg} \mathrm{cm}^{-2} \mathrm{~s}^{-1}$ (Nov 2011/RGS). Thus from the lowest ionization to highest, $\xi$ increases by a factor $\times 2.3$, while the soft X-ray flux increase by the same factor. This would appear to suggest that the low ionization absorber is in photoionization equilibrium with the continuum. In contrast there appears to be no change in the higher ionization components 2 and 3, within the errors. Note that this behavior is also consistent with a December $2002(80 \mathrm{ks})$ Chandra LETG observation (not analyzed here), which was at about a 35\% lower flux than the 2002 HETG observation, but observed the low ionization absorber to have an even lower ionization, of $\log \left(\xi / \mathrm{erg} \mathrm{cm} \mathrm{s}^{-1}\right)=0.63 \pm 0.06$ (Ramírez et al. 2008).

We also illustrate the apparent change in ionization further in Figure11, which plots the change in the xstar model from varying the ionization of warm absorber component 1, against the 2011 RGS data in the Fe M-shell UTA band. The upper panel of Figure11 plots the best fit model obtained, with an ionization parameter of $\log \left(\xi / \mathrm{erg} \mathrm{cm} \mathrm{s}^{-1}\right)=1.27$ for component 1 , as reported in Table5 Then the ionization parameter was lowered (and fixed) to $\log \left(\xi / \mathrm{erg} \mathrm{cm} \mathrm{s}^{-1}\right)=1.15$, equal to the value found for component 1 in the 2011 HETG spectrum. This results in a worse fit, as seen in panel (b) of Figure 11, indeed even allowing the other warm absorber and continuum parameters in the fit to vary resulted in a worse fit by $\Delta \chi^{2}=24.6$. Similarily if the ionization parameter is lowered still further, to $\log \left(\xi / \mathrm{erg} \mathrm{cm} \mathrm{s}^{-1}\right)=0.91$ as obtained from the 2002 Chandra HETG data, the fit is substantially worse by $\Delta \chi^{2}=125.4$, compared to the best fit case shown in panel (a). Indeed this can be seen in panel (c) of Figure 11, whereby the drop in the Fe M-shell UTA region observed at $17.5-18.5 \AA$ is too shallow compared to the data, while the spectrum is then too absorbed red-wards of this feature. Thus overall the Fe M-shell UTA region appears to be quite sensitive to the ionization state of the spectrum.

The other possible change in the spectra is in the partial covering absorption. Considering all three grating observations, the uncovered fraction (or $1-f$ ) of the power-law (in other words the fraction that is not obscured by the partial covering absorption) appears to increase as the flux increases from the 2002 through to the 2011 observations, from $(1-f)=0.18 \pm 0.02$ to $(1-f)=0.39 \pm 0.03$. This may suggest 
that the AGN is more obscured when it is in a lower flux state, which has been claimed in several Seyferts to date (e.g., NGC 3516: Turner et al. 2005, 2008; PG 1211+143: Bachev et al. 2009; Pounds \& Reeves 2009; H 0557-385: Longinotti et al. 2009; NGC 4051: Terashima et al. 2009; Lobban et al. 2011), and indeed variable X-ray absorption was first suggested from soft X-ray band variations in MR 2251-178 itself (Halpern 1984). This variability behaviour will be investigated further in a subsequent paper (Porquet et al. 2013, in preparation), considering a broad-band X-ray analysis of all the contemporary and archival observations of MR 2251-178.

\subsection{Is there a very highly ionized absorber?}

Previous studies of MR 2251-178, with a 2009 Suzaku observation (Gofford et al. 2011) and the 2002 HETG observation (Gibson et al. 2005), have suggested the presence of a highly ionized and possibly strongly outflowing, absorption component in the iron K band. Such absorption could be similar to the very highly ionized outflows (or "ultra fast outflows") detected in about $40 \%$ of local type I AGN with XMM-Newton (Tombesi et al. 2011) and Suzaku (Gofford et al. 2013). Thus we have analyzed the higher energy 2011 Chandra HETG observation above $2 \mathrm{keV}$, using the High Energy Grating (HEG) spectrum, to assess whether such a component is present in the new data. The 2002 HETG spectrum was also re-analyzed for comparison, while the results are also compared to the Suzaku analysis in Gofford et al. (2011).

Figure 12 shows the data/model residuals of the 2011 HEG spectrum to the best-fit absorption model discussed above, plotted over the Fe $\mathrm{K}$ band in the quasar rest frame further binning the spectrum to 20 counts per bin to increase the signal to noise. First we consider the iron $\mathrm{K}$ band emission. The lack of any strong iron $\mathrm{K} \alpha$ emission is quite apparent in the residuals. Indeed the limit on the equivalent width of a narrow $6.4 \mathrm{keV}$ line is $11 \pm 6 \mathrm{eV}$ and is only very marginally required at $\sim 95 \%$ confidence in the fit, with $\Delta C=6.3$. The limit on the width of the line is $\sigma<28 \mathrm{eV}$ or $\sigma<1300 \mathrm{~km} \mathrm{~s}^{-1}$. No other iron $\mathrm{K}$ emission component is required in the spectrum, either narrow or broad. The weakness of the iron $\mathrm{K} \alpha$ line in MR 2251-178 has also been noticed previously (Gofford et al. 2011 and references therein), and is much weaker that the typical narrow iron line equivalent width of $\sim 50-100 \mathrm{eV}$ observed in most Seyfert $1 \mathrm{~s}$ (e.g., Nandra et al. 1997; Patrick et al. 2012; Tatum et al. 2013). The weakness of the iron K line may be accounted for by the X-ray Baldwin effect, whereby the equivalent width of the iron $\mathrm{K} \alpha$ line appears to decrease with AGN X-ray luminosity (e.g., Iwasawa \& Taniguchi 1993; Nandra et al. 1997; Reeves \& Turner 2000; Page et al. 2004; Bianchi et al. 2007; Shu et al. 2010). The 2-10 keV X-ray luminosity of MR 2251178 in this observation is $3.7 \times 10^{44} \mathrm{erg} \mathrm{s}^{-1}$ (or absorption corrected, $5.8 \times 10^{44} \mathrm{erg} \mathrm{s}^{-1}$ ), higher than most local Seyfert $1 \mathrm{~s}$.

There does appear to be a broad but shallow absorption trough in the 2011 data at $7.3 \mathrm{keV}$. Fitting the trough with a Gaussian absorption profile gives a rest frame centroid energy of $E=7.34 \pm 0.08 \mathrm{keV}$ with an equivalent width of $\mathrm{EW}=-58 \pm 24 \mathrm{eV}$ and the fit statistic improves by $\Delta C=15.2$. Note this appears to be consistent with the high energy absorption line that was previously claimed in the 2002 HETG observation

by Gibson et al. (2005); there the line centroid was at $E=7.26 \pm 0.04 \mathrm{keV}$. Furthermore Gofford et al. 
(2011) claimed an absorption trough in the Suzaku observation at an energy of $E=7.57_{-0.12}^{+0.19} \mathrm{keV}$, which is only marginally inconsistent at $90 \%$ confidence with the line energy measured by the 2011 Chandra data, while the equivalent width of $-26_{-12}^{+18} \mathrm{eV}$ is consistent. In Figure 12 the $2002 \mathrm{HEG}$ spectrum has been overlayed on the 2011 data, with the normalization of the 2002 spectrum allowed to vary to account for the overall lower flux level in the 2002 observation, it appears that the trough in the 2002 data has a consistent profile in both energy and depth with the 2011 data. With the 2002 and 2011 fitted together with a single Gaussian profile, then consistent parameters were obtained, with a line energy of $E=7.32 \pm 0.06 \mathrm{keV}$ and an equivalent width of $-60 \pm 18 \mathrm{eV}$. The fit statistic was improved by $\Delta C=26.2$ with respect to a model without the absorption line. The profile appears to be resolved compared to the HETG resolution, with a width of $\sigma=120_{-40}^{+50} \mathrm{eV}$ or $\sigma=4900_{-1600}^{+2100} \mathrm{~km} \mathrm{~s}^{-1}$. Note if the absorption line is associated with the Fe XXVI (H-like) $1 s \rightarrow 2 p$ doublet at $6.97 \mathrm{keV}$, then the velocity shift implied is $-15000 \pm 2600 \mathrm{~km} \mathrm{~s}^{-1}$. We also note that no significant iron $\mathrm{K} \alpha$ emission was required from refitting the $2002 \mathrm{HEG}$ spectrum, although the upper limit to its equivalent width is less well determined $(<40 \mathrm{eV})$ and is consistent with the 2011 measurement.

We attempted to model the Fe $\mathrm{K}$ band absorption with a highly ionized XSTAR grid. Unlike for the warm absorber components, a high turbulence velocity grid was used, with $\sigma=5000 \mathrm{~km} \mathrm{~s}^{-1}$, consistent with the observed line width and an illuminating hard X-ray continuum of $\Gamma=2$. The ionization parameter is not so well constrained, with $\log \left(\xi / \mathrm{erg} \mathrm{cm} \mathrm{s}^{-1}\right)=4.8_{-0.8}^{+1.0}$, but suggests that either H-like or He-like iron contributes to the absorption. The column was found to be largely degenerate upon the ionization parameter (i.e. as the ionization increases the column increases to compensate) and only a lower-limit can be placed of $N_{\mathrm{H}}>1.5 \times 10^{23} \mathrm{~cm}^{-2}$. The outflow velocity derived was consistent with the line analysis, with $v_{\text {out }}=-15600 \pm 2400 \mathrm{~km} \mathrm{~s}^{-1}$ and is consistent with the Gibson et al. (2005) value of $v_{\text {out }}=-12700 \pm$ $2400 \mathrm{~km} \mathrm{~s}^{-1}$. However we also note that at this velocity, the absorption is only marginally excluded at $90 \%$ confidence from being associated from a local $z=0$ absorber.

We also tested whether the iron K-shell region could instead be fitted with a photoelectric edge, from neutral or mildly ionized iron, without any velocity shift as was implied from the highly ionized absorption model. Indeed fitting the Chandra data with a simple edge model results in a equally good fit statistically, with a best fit edge energy of $E=7.15 \pm 0.05 \mathrm{keV}$ and optical depth $\tau=0.15 \pm 0.05$. Such an edge component could plausibly result from a partial covering absorber with column density typically exceeding $N_{\mathrm{H}}>10^{23} \mathrm{~cm}^{-2}$ and as has been discussed, this may also be required from fitting the broader band HETG spectrum. Thus it is not possible to distinguish here between the high velocity absorber and possible partial covering cases in MR 2251-178 and higher resolution data in the Fe K bandpass, such as with the calorimeter to be flown on Astro-H, would be required to differentiate between these cases.

Thus the detection of the Fe $\mathrm{K}$ band absorption trough appears to be confirmed from the two Chandra observations, with the parameters consistent in both and at the same rest frame energy, although its exact origin remains uncertain. Gofford et al. (2011) also claimed further blueshifted absorption features at lower energies from the Suzaku data; in particular absorption lines at $E=2.52 \pm 0.02 \mathrm{keV}$ and $E=2.79 \pm 0.03 \mathrm{keV}$ in the quasar rest frame, which were identified with blueshifted S XV and S XVI $1 s \rightarrow 2 p$ respectively. A $1.3 \mathrm{keV}$ absorption trough was present in the Suzaku data near $1.3 \mathrm{keV}$ and tentatively identified with 
blue-shifted iron L-shell transitions. In the latter case, the much higher resolution HETG and RGS spectra resolve the $1.3 \mathrm{keV}$ absorption into a series of lower ionization lines of inner shell $\mathrm{Mg}$ from $\mathrm{Mg}$ VI-IX, with only a modest outflow velocity of $\sim-400 \mathrm{~km} \mathrm{~s}^{-1}$. However the absorption line at $2.52 \mathrm{keV}$ appears to be only marginally detected in the 2011 Chandra spectrum at $\sim 99 \%$ confidence $(\Delta C=9.3)$ at $E=2.521 \pm 0.002 \mathrm{keV}$ in the quasar rest frame (or $4.92 \AA$ ) with an equivalent width of $-2.0 \pm 1.2 \mathrm{eV}$; these parameters are entirely consistent with those measured by Suzaku. An absorption line is not detected at $2.79 \mathrm{keV}$, however the limit on the equivalent width of $\mathrm{EW}<4 \mathrm{eV}$ from Chandra is consistent with the Suzaku measurement of $-5 \pm 2 \mathrm{eV}$. Thus the presence of this possible higher velocity component appears uncertain based on the current data and such a component does not appear to be present in line profiles of $\mathrm{C}$ through to Si.

\section{Modeling the Emission Line Spectrum}

As we have noted previously, the 2011 RGS and HETG observations contain several soft X-ray emission lines, which have been fitted with simple Gaussian emission line profiles. The parameters of these emission lines are listed in Table 6. Most of the lines were detected in the RGS rather than the HETG, as the RGS has a higher effective area below $1 \mathrm{keV}$. Many of the lines detected are substantially broadened, with typical widths of several thousand $\mathrm{km}^{-1}$, from C VI Lyman- $\alpha, \mathrm{N}$ VI, O VII, and Ne IX. Two weaker narrow components are also present from N VII Lyman- $\alpha$, and Ne IX, with velocity widths typically $\lesssim 1000 \mathrm{~km} \mathrm{~s}^{-1}$ (FWHM). The latter line is detected at an energy of $905 \pm 1 \mathrm{eV}$ in both the RGS and HETG and would appear to be consistent with expected energy of the forbidden line of the Ne IX triplet. As we discuss below, a weak narrow component of the O VII forbidden line cannot be ruled out in the RGS spectrum. Thus it may be plausible that the broad lines originate from BLR type gas, while the narrow (and forbidden) lines originate from gas associated with the NLR.

The O VII line complex is by far the strongest and most statistically significant emission feature detected (with $\Delta \chi^{2}=345.1$ upon its addition to the model), while it also appears be detected with consistent parameters in the HETG spectrum (albeit less well constrained). We therefore concentrate on the analysis of the O VII line complex, using the high signal to noise RGS spectrum. The line complex width is certainly broadened, with a FWHM velocity width of $10200_{-1400}^{+1200} \mathrm{~km} \mathrm{~s}^{-1}$. Note that the width of the C VI line complex is poorly constrained, as it lies at the low energy end of the RGS bandpass, and so has been set equal to the $\mathrm{O}$ VII line complex width, which is the best determined broad line.

An enlarged view of the O VII RGS line complex profile is plotted in Figure 13. Note that this portion of the spectrum only contains data from RGS 1, due to the malfunctioning RGS 2 chip over this energy range. The fit with a single broad line profile is good, with an overall fit statistic of $\chi^{2} /$ dof $=3007.7 / 2564$. It is also apparent that three narrow absorption lines are superimposed upon the emission line profile, which have been identified with inner shell O V-VI, e.g. see Table 3 We tested whether a narrow $(\sigma<1 \mathrm{eV})$ component due to the $\mathrm{O}$ VII forbidden line at $561.0 \mathrm{eV}$ could also be added to the profile and indeed such a component cannot be excluded, with an equivalent width of $\mathrm{EW}=0.9 \pm 0.4 \mathrm{eV}$ and an improvement in 
fit statistic of $\Delta \chi^{2}=16.0$. The equivalent width of the narrow component is much weaker than that of the broad line, which has $E W=8.3_{-1.1}^{+0.9} \mathrm{eV}$ and thus its overall contribution towards the profile is negligible.

Note the energy of the broad O VII emission line is $E=564.5 \pm 0.9 \mathrm{eV}$, which is somewhat blue-shifted compared to the expected energy of the forbidden line at $561.0 \mathrm{eV}$. If the broad emission is purely associated with the forbidden emission, this would suggest an overall blue-shift of $-1900 \pm 500 \mathrm{~km} \mathrm{~s}^{-1}$. Alternatively it may be that the profile consists of a blend of forbidden $(561.0 \mathrm{eV})$, intercombination $(568.6 \mathrm{eV})$ and resonance $(573.9 \mathrm{eV})$ emission. A blend of narrow lines can be ruled out at high confidence, as the fit statistic is substantially worse $\left(\chi^{2} /\right.$ dof $\left.=3135.5 / 2565\right)$ and the majority of the O VII flux is not accounted for. However the profile can be fitted by a blend of velocity broadened lines. In order to test this, the forbidden, intercombination and resonance lines were fitted with line energies fixed at their expected values, with a common velocity width for all 3 line components allowed to vary. This provides an excellent fit to the line profile, with $\chi^{2} /$ dof $=2974.4 / 2563$, while the FWHM width of the 3 lines is now $7300_{-1500}^{+1000} \mathrm{~km} \mathrm{~s}^{-1}$. The parameters of the three line components are listed in Table 6 , while the line model is the one overlaid on the O VII profile in Figure 13. From the line fluxes listed in Table 6, it is apparent that the flux of the forbidden line component dominates over the intercombination emission, while only an upper-limit is placed on the resonance line emission. The dominance of the forbidden line emission over the other components is perhaps expected, as the centroid of the broad O VII profile is closest to the expected forbidden line energy. In section 6.2.1 we attempt to place constraints on the density and location of the emitter given these O VII parameters.

\section{Discussion}

\subsection{Main observational results}

The exposure time of both the HETG and RGS observations allow us to perform an unprecedented high signal-to-noise and high resolution spectroscopy study of the properties of both the primary continuum and the ionized absorption and emission features in the quasar MR 2251-178. The main observational results are the following:

In the soft X-ray range, numerous absorption features are clearly detected:

- A deep absorption trough between $0.7-0.8 \mathrm{keV}$ most likely identified with an unresolved transition array (UTA), due to $2 p \rightarrow 3 d$ transitions from low ionization M-shell iron, i.e. Fe VII-X;

- A multitude of inner $\mathrm{K}$-shell lines of $\mathrm{O}, \mathrm{Ne}, \mathrm{Mg}$ and $\mathrm{Si}$, due to charge states corresponding to $\mathrm{Li}, \mathrm{Be}, \mathrm{B}$, $\mathrm{C}, \mathrm{N}$, O-like etc ions.

- Several higher ionization L-shell ( $2 p \rightarrow 3 d$ ) lines of iron (i.e. Fe XVII-XXIV).

- Resonance $(1 s \rightarrow 2 p)$ lines from $\mathrm{He}$ and $\mathrm{H}$-like ions of $\mathrm{C}, \mathrm{N}, \mathrm{O}, \mathrm{Ne}, \mathrm{Mg}$ and $\mathrm{Si}$, and in some cases, higher order $1 s \rightarrow n p$ lines up to $n=6$.

In most cases the (strongest) absorption line profiles are narrow or not resolved, with velocity widths typically $\sigma \lesssim 300 \mathrm{~km} \mathrm{~s}^{-1}$. Similarly the outflow velocities inferred from the measured rest frame energies of the absorption lines are small or consistent with zero, of the order $v_{\text {out }} \lesssim 400 \mathrm{~km} \mathrm{~s}^{-1}$. 
The spectral fit using photo-ionised XSTAR model grids shows that 3 fully-covering WA components are required in order to model the wide range in the ionization state of the gas, with $N_{\mathrm{H}}=1.5-3.6 \times$ $10^{21} \mathrm{~cm}^{-2}$ and $\log \left(\xi / \mathrm{erg} \mathrm{cm} \mathrm{s}^{-1}\right)=1.27-2.80$. The small outflow velocities found for the low and medium ionization components 1 and 2 are consistent with each other, with $v_{\text {out }}=-480 \pm 40 \mathrm{~km} \mathrm{~s}^{-1}$ and $v_{\text {out }}=-460 \pm 60 \mathrm{~km} \mathrm{~s}^{-1}$ respectively, while the highest ionization component 3 does not require an outflow velocity with $v_{\text {out }}<130 \mathrm{~km} \mathrm{~s}^{-1}$. Notably, the necessary power-law continuum required by the XSTAR grid in order to model the low ionization lines (component 1 ) is $\Gamma_{\text {input }}=2.5$, which is softer than that required for the higher ionized lines (i.e., $\Gamma_{\text {input }}=2.0$ ). Moreover, one additional component of partially (covering factor $\sim 61 \%$ ) ionized absorbing gas with $N_{\mathrm{H}} \sim 6 \times 10^{22} \mathrm{~cm}^{-2}$ and $\log \left(\xi / \mathrm{erg} \mathrm{cm} \mathrm{s}^{-1}\right) \sim 1$ is required to achieve a good fit. Interestingly after the required absorbing layers of gas are accounted for, the soft X-ray photon index found $(\Gamma=2.32 \pm 0.08)$ is in good agreement with what is required to reproduce the soft X-ray inner shell absorption lines (i.e. $\Gamma \sim 2.5$ ). Therefore, MR 2251-178 may have an intrinsically soft continuum, at least below $2 \mathrm{keV}$, which is partially-covered by a complex and stratified absorber.

For the 2011 HETG spectrum the parameters of the three fully-covering WA are rather similar to those obtained from the RGS spectra with $N_{\mathrm{H}}=1.5-2.1 \times 10^{21} \mathrm{~cm}^{-2}$, and $\log \left(\xi / \mathrm{erg} \mathrm{cm} \mathrm{s}^{-1}\right)=1.15-2.9$, but a second partial covering component (covering factor $\sim 40 \%$ ) of higher column density $\sim 7 \times 10^{23} \mathrm{~cm}^{-2}$ seems to be required, from the spectral curvature above $2 \mathrm{keV}$. As for the RGS spectrum, a softer input continuum is strongly required to model the low ionization warm absorber component 1 . However, there is evidence for a small, but significant change in its ionization parameter that appears to be correlated with the soft X-ray flux. Applying this model to the 2002 HETG spectrum we confirm that the change of the ionization parameter is in direct proportion to the soft X-ray flux suggesting that this component is in photoionization equilibrium with the continuum. The other possible change in the spectra is in the partial covering absorption. Considering all three grating observations, the uncovered fraction of the power-law appears to increase as the flux increases from the 2002 through to the 2011 observations, from $0.18 \pm 0.02$ to $0.39 \pm 0.03$, suggesting that this AGN is more obscured at lower flux states.

The soft X-ray spectra also display several emission lines from a photoionized emitter from He- and $\mathrm{H}$-like ions of $\mathrm{C}, \mathrm{N}, \mathrm{O}$, and Ne. Notably, a strong and broad emission line near $0.56 \mathrm{keV}$ is clearly detected in the RGS 1 spectrum at the expected energy of the O VII triplet, and is well represented by a blend of the forbidden (dominant), intercombination and resonance emission lines with a common velocity of $\sim 7300 \mathrm{~km} \mathrm{~s}^{-1}$ (FWHM). This broad O VII triplet profile is superimposed on by three narrow absorption lines corresponding to inner-shell absorption due to $\mathrm{OV}$ and the two lines which make up the $\mathrm{OVI}$ $\left(1 s^{2} 2 s \rightarrow 1 s 2 s 2 p\right)$ doublet. Similar structures are present at other energies, with N VII, O VIII and Ne IX all showing emission superimposed by absorption.

In the hard X-ray energy band of the HETG spectrum, there is a lack of any strong iron $\mathrm{K} \alpha$ emission, with $\mathrm{EW}=11 \pm 6 \mathrm{eV}$. This could be accounted for by the X-ray Baldwin effect, since MR2251-178 has a much higher $2-10 \mathrm{keV}$ luminosity than most local Seyfert 1s. However, we found the presence of a significant absorption feature at $7.3 \mathrm{keV}$ consistent with what was previously reported from the 2002 HETG observation by Gibson et al. (2005), but only marginally inconsistent at $90 \%$ confidence with the line energy measured in the 2009 Suzaku observation by Gofford et al. (2011). This Fe K band absorption is well modelled 
by a highly ionized XSTAR grid with a high turbulence velocity of $5000 \mathrm{~km} \mathrm{~s}^{-1}$ and an outflow velocity of $\sim-15600 \mathrm{~km} \mathrm{~s}^{-1}$. However an alternative origin from a low ionization partial covering absorber without requiring any velocity shift cannot be excluded. The much higher spectral resolution of both HETG and RGS data allows us to resolve the $1.3 \mathrm{keV}$ absorption feature - first observed in the lower resolution 2009 Suzaku XIS spectrum (Gofford et al. 2011) and tentatively identified with blue-shifted iron L-shell transitions - into a series of lower ionization lines of inner shell Mg from Mg VI-IX, with only a modest outflow velocity of $\sim-400 \mathrm{~km} \mathrm{~s}^{-1}$.

\subsection{The Origins of the Warm Absorption and Emission in MR 2251-178}

\subsubsection{Constraints from the $O$ VII line triplet}

Given the constraints on the O VII line triplet, we can attempt to estimate the density and likely radial location of the emitting gas. The line ratios $G=(x+y+z) / w$ and $R=z /(x+y)$ give a measure of the temperature and density of the gas, where $z$ corresponds to the forbidden line, $(x+y)$ to the intercombination emission and $w$ to the resonance line (Porquet \& Dubau 2000). From the line ratios in Table 6 , this yields $G>3.9$ and $R=2.9 \pm 1.4$. Thus from the calculations in Porquet \& Dubau (2000), the high $\mathrm{G}$ ratio corresponds to the gas being photoionized rather than collisionally ionized, with a temperature $T<10^{6} \mathrm{~K}$. However, photo-excitation of the resonance lines can be important in X-ray photoionized sources as AGN (e.g., Kinkhabwala et al. 2002; Porquet et al. 2010), thus other complementary temperature diagnostics should be used such as those based on the width measurement of the recombination continuum (RRC) features (Liedahl \& Paerels 1996). Unfortunately in the spectrum of MR 2251-178, no RRC emission is detected, so it is not possible to determine the temperature by this method.

The $R$ values suggests a density of $n_{\mathrm{e}} \sim 10^{10} \mathrm{~cm}^{-3}$, while the fact that the forbidden line is required to be stronger than the intercombination emission (i.e the lower limit is $R>1.4$ ) implies that the maximum possible density is $<10^{11} \mathrm{~cm}^{-3}$. Thus a density of $n_{\mathrm{e}}=10^{10}-10^{11} \mathrm{~cm}^{-3}$ would seem to imply an origin of the broad line emission consistent with the optical Broad Line Region (Davidson \& Netzer 1979). The ionization of the emitter can also be constrained, given that a line flux ratio of $\mathrm{O}$ VII/O VIII $\sim 6$, e.g. Table 6. From running an XSTAR simulation with a density of $n_{\mathrm{e}}=10^{10} \mathrm{~cm}^{-3}$, the line ratio implies an ionization parameter of $\log \left(\xi / \mathrm{erg} \mathrm{cm} \mathrm{s}^{-1}\right)=1.25$. Thus an estimate of the radial distance can be obtained via the definition of the ionization parameter, i.e. $r=\left(L_{\text {ion }} / \xi n_{\mathrm{H}}\right)^{1 / 2}$, where $L_{\text {ion }}$ is the $1-1000$ Rydberg luminosity and $n_{\mathrm{H}}$ is the hydrogen number density. From extrapolating the best-fit spectrum from above, the ionizing luminosity of MR 2251-178 is $L_{\mathrm{ion}}=2 \times 10^{45} \mathrm{erg} \mathrm{s}^{-1}$. Thus for a density in the range $n_{\mathrm{e}}=$ $10^{10}-10^{11} \mathrm{~cm}^{-3}$, then the radius is $r=0.3-1.0 \times 10^{17} \mathrm{~cm}$ (or $0.01-0.03 \mathrm{pc}$ ), again consistent with typical BLR radii (e.g., Kaspi et al. 2005). The radius of the emission can also be estimated from the O VII width of $\sigma=3200 \mathrm{~km} \mathrm{~s}^{-1}$. Assuming a virial relation between the black hole mass and the radius $r$, of $3 \sigma^{2}=G M / R$ (Peterson et al. 2004) and adopting a black hole mass of $2.4 \times 10^{8} \mathrm{M}_{\odot}$ for MR 2251-178 (Dunn et al. 2008), gives a radius of $r \sim 10^{17} \mathrm{~cm}$, consistent with the above estimate.

Given the estimate of the ionization parameter of the soft X-ray emitter of $\log \xi=1.25$, it can plausibly 
be associated with one of two absorption components, either the low ionization warm absorber component 1 or the partial covering component (pc1), as summarised in Table 5] We have calculated the total (global) covering fraction as a fraction of $4 \pi \mathrm{sr}\left(f_{\text {tot }}\right)$ for either absorbing layer in order to produce the total luminosity of the broad O VII emission, of $3.1 \times 10^{42} \mathrm{erg} \mathrm{s}^{-1}$. The XSTAR code is used to calculate the line luminosity from a spherical shell of gas, covering a full $4 \pi \mathrm{sr}$ around the AGN, illuminating by the above ionizing luminosity. The component 1 absorber has a column density of $N_{\mathrm{H}}=2 \times 10^{21} \mathrm{~cm}^{-2}$ and produces an O vII luminosity over $4 \pi$ sr of $3.7 \times 10^{42} \mathrm{erg} \mathrm{s}^{-1}$, while the partial covering component has $N_{\mathrm{H}}=5 \times 10^{22} \mathrm{~cm}^{-2}$ and produces an $\mathrm{O}$ VII luminosity of $4.5 \times 10^{43} \mathrm{erg} \mathrm{s}^{-1}$. Thus in order to reproduce the $\mathrm{O}$ VII luminosity, the component 1 absorber would require a high covering fraction of $f_{\text {tot }}=0.84$ of $4 \pi \mathrm{sr}^{-1}$, while the partial coverer only requires a fraction of $f_{\text {tot }}=0.07$.

However some of the narrow absorption lines that are produced from the component 1 warm absorber itself are superimposed upon the O VII broad emission profile. This would appear to require the component 1 absorber to be physically placed outside the line emitting region, making it less likely to be the origin of the broad soft X-ray lines. Furthermore the kinematics of component 1, with a low outflow velocity $\left(\sim-400 \mathrm{~km} \mathrm{~s}^{-1}\right)$ and small or unresolved line widths/turbulences, would also suggest it is placed at larger distances perhaps coincident with the NLR. Therefore one possibility is that the broad line region clouds themselves not only produce the broad soft X-ray lines, but are also responsible for the partial covering of the X-ray continuum itself. Such broad X-ray emission ionized lines have been detected in several other AGN thanks to high-resolution X-ray data suggesting that such a BLR origin for the X-ray emission may be common in AGN (e.g., Mrk 279: Costantini et al. 2007, Mrk 841: Longinotti et al. 2010, NGC 4051: Ogle et al. 2004, Mrk 509: Detmers et al. 2011, 3C 445: Reeves et al. 2010).

Note that the estimate of the total covering fraction, of $\sim 7 \%$ for the partial coverer/emitter, may be substantially higher if some of the broad line emission is itself obscured, depending on the exact spatial distribution of emitting and absorbing clouds. We note that in the RGS data about $60 \%$ of the intrinsic $\mathrm{X}$-ray continuum is obscured by the $\sim 5 \times 10^{22} \mathrm{~cm}^{-2}$ partial coverer (40\% remains unobscured). If this obscuration is also applied to the broad O VII emission, that may imply a total covering fraction of the emitting clouds closer to $f_{\text {tot }} \sim 0.2$. Furthermore the X-ray BLR emission can be further obscured by the warm absorber which fully covers the line of sight to the AGN, which obscures the continuum level by a factor of about 30-40\% at the energy of the O VII emission line. Thus the total covering is likely to be consistent with typical estimate of the overall covering fraction of optical BLR clouds, of the order $5-30 \%$ (e.g., Netzer \& Laor 1993). If the BLR clouds do partially cover the X-ray source, then this can give an approximate estimate of a size of a cloud. Thus for X-ray absorption of the order $\sim 10^{23} \mathrm{~cm}^{-2}$ and for a density of $n_{\mathrm{e}} \sim 10^{10} \mathrm{~cm}^{-3}$, then that implies a size of $\Delta r \sim 10^{13} \mathrm{~cm}$, likely smaller than the size of the $\mathrm{X}$-ray emission region (e.g. $10 R_{\mathrm{g}}$ here would correspond to a few $\times 10^{14} \mathrm{~cm}$ ). Thus it seems plausible for such clouds to only partially cover the line of sight to the continuum X-ray emission.

The low ionization component 1 warm absorber could instead plausibly reproduce some of the weak narrow emission lines in the spectrum, e.g. the narrow forbidden components, which have line widths of $\sigma \lesssim 500 \mathrm{~km} \mathrm{~s}^{-1}$ typically. This would correspond to radial distances of a few pc or greater. The distance to the component 1 absorber is estimated below, via its response to the soft X-ray continuum. 


\subsubsection{Constraints from the variability of the warm absorber components}

The component 1 warm absorber appears to respond to the overall increase in the continuum between the 2002 and 2011 observations, but also in the $\sim 40$ days timescale between the 2011 XMM-Newton and Chandra observations (Section 4.2) and thus would appear to be in photoionization equilibrium. We can therefore attempt to place a lower limit on the density of this absorber via the recombination timescale. For this we use the recombination time-scale formula from Bottorff et al. (2000) that does account simultaneously for the cascade into the population of $X_{i}$ ions from the population of $X_{i+1}$ ions, and the cascade out of the population of $X_{i}$ ions into the population of $X_{i-1}$ ions:

$$
t\left(X_{i}\right)=\frac{1}{\alpha\left(X_{i}\right) n_{e}\left[\frac{f\left(X_{i+1}\right)}{f\left(X_{i}\right)}-\frac{\alpha\left(X_{i-1}\right)}{\alpha\left(X_{i}\right)}\right]} .
$$

where $f\left(X_{i}\right)$ the ionic fraction of the $X_{i}$ ion, $\alpha\left(X_{i}, T_{e}\right)$ is the recombination coefficient of the $X_{i}$ ion at the electronic temperature $T_{e}$, and $n_{e}$ is the electron density ( $\sim 1.2 n_{H}$ for cosmic abundance). We apply this formula to $\mathrm{O}$ VII. At $\log \xi=1.27$, the ratio $\mathrm{O}$ VII/O VIII is 6.0 and $T_{e}$ is $4 \times 10^{4} \mathrm{~K}$. Using the recombination coefficient from Nahar \& Pradhan (2003) and a recombination time of $t \lesssim 40$ days between observations, we find a lower limit for the hydrogen density of $3.8 \times 10^{4} \mathrm{~cm}^{-3}$. Hence, this implies an upper limit for the radial distance $\left(R_{\mathrm{var}}\right)$ of $5.3 \times 10^{19} \mathrm{~cm}$ (i.e. $\lesssim 17 \mathrm{pc}$ ) or a few pc. Moreover as discussed below in 6.2 .3 the minimum radius for component 1 is about $2.8 \times 10^{19} \mathrm{~cm}$ (i.e. $\gtrsim 9 \mathrm{pc}$ ). Therefore the location of component 1 is well constrained between $9 \mathrm{pc}$ and $17 \mathrm{pc}$. For comparison, the expected distances of the torus and of the NLR that are about $7 \mathrm{pc}$ and about $140 \mathrm{pc}$, using the following formula of Krolik \& Kriss (2001) and Mor et al. (2009) respectively:

$$
\begin{gathered}
R_{\text {torus }} \sim L_{\text {ion, } 44}^{1 / 2} \quad(p c) \\
R_{\mathrm{NLR}}=295 \times L_{46}^{0.47 \pm 0.13} \quad(p c) .
\end{gathered}
$$

For MR 2251-178 the ionizing (1-1000 Rydberg) luminosity was taken as $L_{\text {ion, } 44}=20$ (in units of $10^{44} \mathrm{erg} \mathrm{s}^{-1}$ ) and $L_{46}=0.434$ is assumed as the bolometric luminosity (Dunn et al. 2008), in units of $10^{46} \mathrm{erg} \mathrm{s}^{-1}$.

Therefore component 1 appears to be located consistent with the pc scale torus and/or inner NLR radius-scales. We note that $R_{\mathrm{var}}$ is much greater than the BLR distance that is of only about 75 light-days (using the recent $\mathrm{R}_{\mathrm{BLR}}-\lambda L_{\lambda}(5100 \AA$ ) relationship from Bentz et al. 2013 and the average $5100 \AA$ flux from Lira et al. 2011), i.e. 0.06 pc. The lack of response from $2002-2011$ of the higher ionization (components 2,3 ) absorbers may place this gas at greater distances. However a more intense monitoring campaign (over weeks to months) would be needed to place a firmer constraint on the density and therefore radial location of the absorbers.

\footnotetext{
${ }^{4}$ This is is likely to be a somewhat conservative estimate of the bolometric luminosity. Applying a bolometric correction of a factor of 30 for the 2-10 keV X-ray luminosity (Vasudevan \& Fabian 2009), would result in $L_{\mathrm{bol}}=10^{46} \mathrm{erg} \mathrm{s}^{-1}$.
} 


\subsubsection{Warm absorber properties: radii, outflows rates and energetics}

We estimate the lower and upper limits of the distance, mass outflow rate and kinetic power of the WAs following the assumptions and definitions outlined in Tombesi et al. (2013) for the fully covering warm absorbers (components 1, 2, and 3) and for the highly ionized absorber discussed in 4.4 An upper limit for the radial location of an absorber can be derived from the definition of the ionization parameter and the requirement that the thickness of the absorber does not exceed its distance to the supermassive black hole, i.e., $N_{\mathrm{H}} \simeq n_{\mathrm{H}} \Delta R<n_{\mathrm{H}} R$, then:

$$
r_{\max } \equiv L_{\text {ion }} / \xi N_{\mathrm{H}}
$$

Note the material can not be farther away than this given the observed ionization and column. An estimate of the minimum distance can be derived from the radius at which the observed velocity corresponds to the escape velocity:

$$
r_{\min } \equiv 2 G M_{\mathrm{BH}} / v_{\text {out }}^{2} .
$$

Here the black hole mass estimate for MR 2251-178 is taken as $2.4 \times 10^{8} M_{\odot}$ (Dunn et al. 2008).

For the calculation of the mass outflow rate we use the expression derived by Krongold et al. (2007) that is appropriate for a biconical wind-like geometry and that does not rely on the estimate of the covering and filling factors (see Tombesi et al. 2013 for details):

$$
\dot{M}_{\text {out }} \equiv f(\delta, \phi) \pi \frac{n_{\mathrm{H}}}{n_{\mathrm{e}}} m_{\mathrm{p}} N_{\mathrm{H}} v_{\text {out }} r
$$

where $f(\delta, \phi)$ is a function that depends on the angle between the line of sight to the central source and the accretion disc plane, $\delta$, and the angle formed by the wind with the accretion disc, $\phi$ (see Fig. 12 of Krongold et al. 2007). As in Krongold et al. (2007) and Tombesi et al. (2013), we assume $f(\delta, \phi) \simeq 1.5$ that corresponds to a roughly vertical disc wind $(\phi \simeq \pi / 2)$ and an average line of sight angle of $\delta \simeq 30^{\circ}$ for a type-I AGN, while $n_{\mathrm{H}} / n_{\mathrm{e}}$ is about $1 / 1.2$ for Solar elemental abundances, so:

$$
\dot{M}_{\text {out }} \simeq 6.6 \times 10^{-24} N_{\mathrm{H}} v_{\text {out }} r \quad[g / s] .
$$

To determine the $\dot{M}_{\text {out }}$ interval range, we use the values of $r_{\max }$ and $r_{\min }$ inferred from equations (5) and (6), except for component 1 for which with use as $r_{\max }$ the value found above due to the recombination time-scale of $\mathrm{O}$ viI, i.e. $r_{\text {var }}$ (see values reported in Table 7).

Neglecting additional acceleration of the outflow, i.e. assuming that it has reached a constant terminal velocity, the kinetic (or mechanical) power can consequently be derived as:

$$
\dot{E}_{\mathrm{K}} \equiv \frac{1}{2} \dot{M}_{\text {out }} v_{\text {out }}^{2}
$$

We also calculated the outflow momentum rate as $\dot{P}_{\text {out }} \equiv \dot{M}_{\text {out }} v_{\text {out }}$ and subsequently compared it to the momentum flux of the radiation field, $\dot{P}_{\text {rad }} \equiv L_{\text {bol }} / c$. All values are reported in Table 7

The inner and outer radii of component 1 are the best determined, between $9-17 \mathrm{pc}$, with the upper bound being set by the 40 day timescale response of the absorber to the continuum. The higher ionization 
component 3 is constrained between between $\sim 120-290 \mathrm{pc}$; this is consistent with being placed outside component 1 , noting that no response of this absorber was detected to continuum variations, consistent with a lower density. Component 2 is the least well determined and is consistent with the radial estimates for components 1 and 3 (Table 7). Thus the locations of components 1 and 3 are consistent with the torus and NLR respectively, as estimated above. The possible highly ionized (iron K band) absorber (Table 7, component high), with an outflow velocity of $\sim 15000 \mathrm{~km} \mathrm{~s}^{-1}$, would appear to be located much closer to the black hole $(\lesssim 0.01 \mathrm{pc})$ with a location perhaps consistent with an accretion disk wind (Tombesi et al. 2013).

The kinetic power of the three warm absorbers (components 1-3) appear to be $\lesssim 0.01 \%$ of the bolometric luminosity; while for the highly ionized absorber we found a minimum value of $1 \%$ of the bolometric luminosity, hence its mechanical power can potentially affect the host galaxy via feedback (Hopkins \& Elvis 2010). Nonetheless the mass outflow rates of all components 1-3 as well as the highly ionized absorber are rather similar, the lower limits on $\dot{M}_{\text {out }}$ vary between $0.2-1.9 M_{\odot} \mathrm{yr}^{-1}$ for components $1-3$, while for the highly ionized absorber, $\dot{M}_{\text {out }} \gtrsim 0.6 M_{\odot} \mathrm{yr}^{-1}$. In comparison for a bolometric luminosity of $4.3 \times 10^{45} \mathrm{erg} \mathrm{s}^{-1}$ and assuming an accretion efficiency of $\eta=0.06$, the expected mass accretion rate of MR 2251-178 is $\dot{M}_{\text {acc }} \sim 1.3 M_{\odot} \mathrm{yr}^{-1}$; thus the combined mass outflow rate from MR 2251-178 is likely to be at least equal to (or somewhat exceeding) the accretion rate onto the black hole. Finally the outward momentum rate of the putative highly ionized absorber is estimated to be at least $\sim 50 \%$ of $L_{\mathrm{bol}} / c$, which suggests efficient $(\tau \sim 1)$ scattering between photons and electrons in a Thomson scattering driven outflow, as may be expected in a highly ionized accretion disk wind (King \& Pounds 2003).

\subsection{Comparisons with UV observations}

Ultra-violet absorption has also been found previously in the spectrum of MR 2251-178. Using HST/FOS data obtained in 1996, Monier et al. (2001) found absorption lines due to Ly $\alpha$, N V and C IV with a systematic blueshift of $\sim 300 \mathrm{~km} \mathrm{~s}^{-1}$ with a total hydrogen column density of about $5 \times 10^{21} \mathrm{~cm}^{-2}$. From the comparison between HST data taken with FOS in 1996 and STIS in 2000, the C IV absorption in particular showed variability - both in terms of the velocity and column density - over a period of roughly 4 years. This relatively short timescale variability showed that this UV absorption is truly intrinsic and constrained the absorption clouds to within $r \lesssim 2.4 \mathrm{kpc}$ of the continuum source (Ganguly et al. 2001) consistent with the estimate of Monier et al. (2001). Kaspi et al. (2004) reported for the first time the entire FUSE spectrum of MR 2251-178 and detected at least four blueshifted absorption systems of C III, H I, and O VI; one at $-580 \mathrm{~km} \mathrm{~s}^{-1}$, and at least three others blended components with centroid velocities at about $-150,-300$, and $-430 \mathrm{~km} \mathrm{~s}^{-1}$.

We note that the velocity profiles obtained here from the X-ray data, e.g. from C VI and O VIII, appear to be consistent with these UV profiles, with the X-ray absorption line profiles having typical velocity shifts of the order $\sim-300--400 \mathrm{~km} \mathrm{~s}^{-1}$, as shown in Figure 7 The only exception may be from the highest ionization lines, such as Mg XII and Si XIV, which do not require any net blue-shift, but this very highly 
ionized gas may be more apparent in the X-ray spectrum than in the UV. The total depth of the FUV and UV absorption lines appeared larger than the underlying continuum, which indicates that the broad UV emission lines are absorbed by the UV absorber and therefore the UV absorber lies outside the BLR. This is similar to what is found in the X-ray spectrum presented here, whereby the broad soft X-ray lines (BLR) are absorbed by the narrow lines from the X-ray warm absorber.

The above UV absorber properties are similar to that found here for the fully-covered soft X-ray warm absorber components (namely components 1,2, and 3), indeed both the column densities and the outflow velocities appear consistent. Moreover, the relatively tight constraint for the location of component 1 shows that it lies outside the BLR region too, with the components 2 and 3 consistent with being further out due to their lack of variability. In conclusion the UV and soft X-ray warm absorption components cover a similar range of column densities and appear to be kinematically consistent with each over in terms of their outflow velocities, although the X-ray absorption likely originates from more highly ionized gas. A more detailed comparison with the 2011 Chandra and XMM-Newton observations and a contemporaneous 2011 HST/COS observation will be deferred until future work. We note however that from a preliminary analysis of the HST/COS spectrum as well as optical spectroscopy (M. Crenshaw, private communication), the FWHM widths of the $\mathrm{C}$ IV and $\mathrm{H} \beta$ emission lines appear in the range $3200-3600 \mathrm{~km} \mathrm{~s}^{-1}$. This is similar to, if somewhat smaller than the widths of the X-ray emission lines such as O VII, with FWHM $\sim 7300 \mathrm{~km} \mathrm{~s}^{-1}$. This could suggest that the broad X-ray lines originate from the innermost part of the BLR, which would likely be more highly ionized.

\subsection{Comparison with the recent observations of Markarian 509}

The Seyfert 1 galaxy Mrk 509 ( $\mathrm{z}=0.03450)$ - which has a similar black hole mass $\left(1-3 \times 10^{8} \mathrm{M}_{\odot}\right)$ and a bolometric luminosity of only a factor of about 2-3 smaller than that of MR 2251-178 (Raimundo et al. 2012) - has also been recently monitored (in 2009) from UV to hard X-rays (HST/COS, XMM-Newton, Chandra, Swift and Integral) to constrain the location of the outflow components (Kaastra et al. 2011b). Thus a comparison between MR 2251-178 and Mrk 509 may be informative, given their similar properties at the higher luminosity end of the Seyfert population, while both AGN have long XMM-Newton or Chandra exposures. The deep $(600 \mathrm{ks})$ XMM-Newton/RGS spectrum of Mrk 509 revealed the presence of a multitude of blueshifted absorption lines from three slow velocity absorber components $\left(\sim-13 \mathrm{~km} \mathrm{~s}^{-1}, \sim-320 \mathrm{~km} \mathrm{~s}^{-1}\right.$, and $\left.-770 \mathrm{~km} \mathrm{~s}^{-1}\right)$, with two strong and discrete ionization parameter peaks in the $\log \left(\xi / \mathrm{erg} \mathrm{cm} \mathrm{s}^{-1}\right)=1-3$ range at about $\log \left(\xi / \mathrm{erg} \mathrm{cm} \mathrm{s}^{-1}\right)=2.0$ and $\log \left(\xi / \mathrm{erg} \mathrm{cm} \mathrm{s}^{-1}\right)=2.8$ (Detmers et al. 2011). The ionization parameters of the UV components with similar outflow velocities are much lower than those found in Xrays, which could indicate that the UV and X-ray absorbers are co-spatial but have different densities, as also inferred from the LETG spectrum (Ebrero et al. 2011). The presence of a possible fast outflow with $v_{\text {out }} \simeq$ $14000 \mathrm{~km} \mathrm{~s}^{-1}$ was claimed using the summed spectrum of previous XMM-Newton observations (Ponti et al. 2009), and was only marginally detected in the LETG 2009 observation and the XMM-Newton/pn spectrum (Ponti et al. 2013). 
The location of the outflowing components in Mrk 509 are claimed to be consistent with a torus wind or NLR origin (Kaastra et al. 2012). While the kinetic luminosity of the outflow is small in Mrk 509, the mass carried away is larger than the likely $0.5 M_{\odot} \mathrm{yr}^{-1}$ accreting onto the black hole. These properties appear to be similar to those presented here for MR 2251-178. Observationally the X-ray column densities, outflow velocities and ionization parameters cover a very similar range, while in terms of radial location, the warm absorbers of both AGN appear commensurate with a pc scale wind consistent with the outermost torus or inner NLR.

\section{Conclusions}

This paper has presented deep (400 ks) Chandra HETG and XMM-Newton RGS observations of the nearby quasar, MR 2251-178. The high resolution spectra have revealed the presence of a three ionization component warm absorber, with the ionization parameter covering the range from $\log \left(\xi / \mathrm{erg} \mathrm{cm} \mathrm{s}^{-1}\right)=$ $1-3$. The lowest ionization component is responsible for the absorption seem from the Fe M-shell UTA as well as the inner-shell lines of $\mathrm{O}, \mathrm{Ne}, \mathrm{Mg}$ and $\mathrm{Si}$, while the higher ionization components produces the $\mathrm{He}$ and $\mathrm{H}$-like lines as well as $\mathrm{L}$-shell $\mathrm{Fe}$ ions. The lowest ionization gas tentatively appears to be in photoionization equilibrium with the continuum flux. From this and from the lower and upper-limits to the radial location of the gas, the low ionization absorber appears consistent with a pc scale location, coincident with either the torus or innermost NLR, while the highest ionization component may arise from more distant gas. The outflow velocities of the warm absorbing gas all appear within $\lesssim 500 \mathrm{~km} \mathrm{~s}^{-1}$, also consistent with the outflow velocities of the known UV absorber in this AGN (Ganguly et al. 2001; Monier et al. 2001; Kaspi et al. 2004).

Several broad emission lines also appeared to be present in the soft X-ray spectrum, most notably from O vII. The width derived for the broad O VII line complex, of FWHM $7300_{-1500}^{+1000} \mathrm{~km} \mathrm{~s}^{-1}$, is consistent with an origin on sub-pc scales from the optical BLR. In addition, we have suggested that the BLR clouds themselves, which are presumably responsible for the broad soft X-ray emission lines, may indeed partially cover the X-ray continuum, with a typical column density of $N_{\mathrm{H}}=10^{23} \mathrm{~cm}^{-2}$. The presence of such a partial coverer has also been recently invoked to account for the hard X-ray excesses observed towards several type I AGN (Tatum et al. 2013) and may be required here to explain the unusually hard X-ray continuum (with $\Gamma=1.5$ ) that is observed in MR 2251-178. Overall the X-ray observations of MR 2251-178 have revealed a complex and stratified absorption and emission region, which modify the overall X-ray spectrum. These appear to exist on several spatial scales; from a putative accretion disc wind responsible for the highly ionized Fe K band absorption; to the BLR clouds responsible for the broad soft X-ray emission lines and potentially the partial covering absorption; and to the more extended outflowing gas on parsec and NLR scales. The latter is the likely origin of the historical soft X-ray warm absorber observed towards this AGN. 


\section{Acknowledgements}

J.N. Reeves acknowledges Chandra grant number GO1-12143X. D. Porquet acknowledges financial support from the French GDR PCHE. T.J. Turner acknowledges NASA grant number AR2-13006X. We would also like to thank Margherita Giustini for helpful discussions. Based on observations obtained with the XMM-Newton, and ESA science mission with instruments and contributions directly funded by ESA member states and the USA (NASA). The scientific results reported in this article are based on observations

made by the Chandra X-ray Observatory. This research has made use of software provided by the Chandra $\mathrm{X}$-ray Center (CXC) in the application packages CIAO, ChIPS.

\section{REFERENCES}

Andrade-Velázquez, M., Krongold, Y., Elvis, M., Nicastro, F., Brickhouse, N., Binette, L., Mathur, S., \& Jiménez-Bailón, E. 2010, ApJ, 711, 888

Antonucci, R. 1993, ARA\&A, 31, 473

Ashton, C. E., Page, M. J., Blustin, A. J., Puchnarewicz, E. M., Branduardi-Raymont, G., Mason, K. O., Córdova, F. A., \& Priedhorsky, W. C. 2004, MNRAS, 355, 73

Bachev, R., Grupe, D., Boeva, S., Ovcharov, E., Valcheva, A., Semkov, E., Georgiev, T., \& Gallo, L. C. 2009, MNRAS, 399, 750

Badnell, N. R. 2006, ApJ, 651, L73

Baumgartner, W. H., Tueller, J., Markwardt, C., \& Skinner, G. 2010, in Bulletin of the American Astronomical Society, Vol. 42, AAS/High Energy Astrophysics Division \#11, 675

Behar, E. \& Netzer, H. 2002, ApJ, 570, 165

Behar, E., Rasmussen, A. P., Blustin, A. J., Sako, M., Kahn, S. M., Kaastra, J. S., Branduardi-Raymont, G., \& Steenbrugge, K. C. 2003, ApJ, 598, 232

Behar, E., Sako, M., \& Kahn, S. M. 2001, ApJ, 563, 497

Bentz, M. C., Denney, K. D., Grier, C. J., Barth, A. J., Peterson, B. M., Vestergaard, M., Bennert, V. N., Canalizo, G., De Rosa, G., Filippenko, A. V., Gates, E. L., Greene, J. E., Li, W., Malkan, M. A., Pogge, R. W., Stern, D., Treu, T., \& Woo, J.-H. 2013, ApJ, 767, 149

Bergeron, J., Dennefeld, M., Boksenberg, A., \& Tarenghi, M. 1983, MNRAS, 202, 125

Bianchi, S., Guainazzi, M., Matt, G., \& Fonseca Bonilla, N. 2007, A\&A, 467, L19

Blustin, A. J., Branduardi-Raymont, G., Behar, E., Kaastra, J. S., Kahn, S. M., Page, M. J., Sako, M., \& Steenbrugge, K. C. 2002, A\&A, 392, 453 
Blustin, A. J., Kriss, G. A., Holczer, T., Behar, E., Kaastra, J. S., Page, M. J., Kaspi, S., BranduardiRaymont, G., \& Steenbrugge, K. C. 2007, A\&A, 466, 107

Blustin, A. J., Page, M. J., Fuerst, S. V., Branduardi-Raymont, G., \& Ashton, C. E. 2005, A\&A, 431, 111

Bottorff, M. C., Korista, K. T., \& Shlosman, I. 2000, ApJ, 537, 134

Braito, V., Reeves, J. N., Dewangan, G. C., George, I., Griffiths, R. E., Markowitz, A., Nandra, K., Porquet, D., Ptak, A., Turner, T. J., Yaqoob, T., \& Weaver, K. 2007, ApJ, 670, 978

Canizares, C. R., Davis, J. E., Dewey, D., Flanagan, K. A., Galton, E. B., Huenemoerder, D. P., Ishibashi, K., Markert, T. H., Marshall, H. L., McGuirk, M., Schattenburg, M. L., Schulz, N. S., Smith, H. I., \& Wise, M. 2005, PASP, 117, 1144

Canizares, C. R., McClintock, J. E., \& Ricker, G. R. 1978, ApJ, 226, L1

Chartas, G., Brandt, W. N., Gallagher, S. C., \& Garmire, G. P. 2002, ApJ, 579, 169

Cooke, B. A., Ricketts, M. J., Maccacaro, T., Pye, J. P., Elvis, M., Watson, M. G., Griffiths, R. E., Pounds, K. A., McHardy, I., Maccagni, D., Seward, F. D., Page, C. G., \& Turner, M. J. L. 1978, MNRAS, 182,489

Costantini, E., Kaastra, J. S., Arav, N., Kriss, G. A., Steenbrugge, K. C., Gabel, J. R., Verbunt, F., Behar, E., Gaskell, C. M., Korista, K. T., Proga, D., Quijano, J. K., Scott, J. E., Klimek, E. S., \& Hedrick, C. H. 2007, A\&A, 461, 121

Crenshaw, D. M. \& Kraemer, S. B. 2012, ApJ, 753, 75

Crenshaw, D. M., Kraemer, S. B., \& George, I. M. 2003, ARA\&A, 41, 117

Davidson, K. \& Netzer, H. 1979, Reviews of Modern Physics, 51, 715

den Herder, J. W., Brinkman, A. C., Kahn, S. M., Branduardi-Raymont, G., Thomsen, K., Aarts, H., Audard, M., Bixler, J. V., den Boggende, A. J., Cottam, J., Decker, T., Dubbeldam, L., Erd, C., Goulooze, H., Güdel, M., Guttridge, P., Hailey, C. J., Janabi, K. A., Kaastra, J. S., de Korte, P. A. J., van Leeuwen, B. J., Mauche, C., McCalden, A. J., Mewe, R., Naber, A., Paerels, F. B., Peterson, J. R., Rasmussen, A. P., Rees, K., Sakelliou, I., Sako, M., Spodek, J., Stern, M., Tamura, T., Tandy, J., de Vries, C. P., Welch, S., \& Zehnder, A. 2001, A\&A, 365, L7

Detmers, R. G., Kaastra, J. S., Steenbrugge, K. C., Ebrero, J., Kriss, G. A., Arav, N., Behar, E., Costantini, E., Branduardi-Raymont, G., Mehdipour, M., Bianchi, S., Cappi, M., Petrucci, P., Ponti, G., Pinto, C., Ratti, E. M., \& Holczer, T. 2011, A\&A, 534, A38

Dunn, J. P., Crenshaw, D. M., Kraemer, S. B., \& Trippe, M. L. 2008, AJ, 136, 1201

Ebrero, J., Costantini, E., Kaastra, J. S., Detmers, R. G., Arav, N., Kriss, G. A., Korista, K. T., \& Steenbrugge, K. C. 2010, A\&A, 520, A36 
Ebrero, J., Kriss, G. A., Kaastra, J. S., Detmers, R. G., Steenbrugge, K. C., Costantini, E., Arav, N., Bianchi, S., Cappi, M., Branduardi-Raymont, G., Mehdipour, M., Petrucci, P. O., Pinto, C., \& Ponti, G. 2011, A\&A, 534, A40

Elvis, M. 2000, ApJ, 545, 63

Ferland, G. J., Korista, K. T., Verner, D. A., Ferguson, J. W., Kingdon, J. B., \& Verner, E. M. 1998, PASP, 110,761

Gallo, L. C., Boller, T., Brandt, W. N., Fabian, A. C., \& Vaughan, S. 2004, A\&A, 417, 29

Ganguly, R., Charlton, J. C., \& Eracleous, M. 2001, ApJ, 556, L7

Gibson, R. R., Marshall, H. L., Canizares, C. R., \& Lee, J. C. 2005, ApJ, 627, 83

Gofford, J., Reeves, J. N., Tombesi, F., Braito, V., Turner, T. J., Miller, L., \& Cappi, M. 2013, MNRAS, 430, 60

Gofford, J., Reeves, J. N., Turner, T. J., Tombesi, F., Braito, V., Porquet, D., Miller, L., Kraemer, S. B., \& Fukazawa, Y. 2011, MNRAS, 414, 3307

Grevesse, N. \& Sauval, A. J. 1998, ssr, 85, 161

Halpern, J. P. 1984, ApJ, 281, 90

Holczer, T. \& Behar, E. 2012, ApJ, 747, 71

Hopkins, P. F. \& Elvis, M. 2010, MNRAS, 401, 7

Iwasawa, K. \& Taniguchi, Y. 1993, ApJ, 413, L15

Kaastra, J. S., de Vries, C. P., Steenbrugge, K. C., Detmers, R. G., Ebrero, J., Behar, E., Bianchi, S., Costantini, E., Kriss, G. A., Mehdipour, M., Paltani, S., Petrucci, P.-O., Pinto, C., \& Ponti, G. 2011 a, A\&A, 534, A37

Kaastra, J. S., Detmers, R. G., Mehdipour, M., Arav, N., Behar, E., Bianchi, S., Branduardi-Raymont, G., Cappi, M., Costantini, E., Ebrero, J., Kriss, G. A., Paltani, S., Petrucci, P.-O., Pinto, C., Ponti, G., Steenbrugge, K. C., \& de Vries, C. P. 2012, A\&A, 539, A117

Kaastra, J. S., Mewe, R., Liedahl, D. A., Komossa, S., \& Brinkman, A. C. 2000, A\&A, 354, L83

Kaastra, J. S., Petrucci, P.-O., Cappi, M., Arav, N., Behar, E., Bianchi, S., Bloom, J., Blustin, A. J., Branduardi-Raymont, G., Costantini, E., Dadina, M., Detmers, R. G., Ebrero, J., Jonker, P. G., Klein, C., Kriss, G. A., Lubiński, P., Malzac, J., Mehdipour, M., Paltani, S., Pinto, C., Ponti, G., Ratti, E. M., Smith, R. A. N., Steenbrugge, K. C., \& de Vries, C. P. 2011b, A\&A, 534, A36

Kaastra, J. S., Steenbrugge, K. C., Raassen, A. J. J., van der Meer, R. L. J., Brinkman, A. C., Liedahl, D. A., Behar, E., \& de Rosa, A. 2002, A\&A, 386, 427 
Kalberla, P. M. W., Burton, W. B., Hartmann, D., Arnal, E. M., Bajaja, E., Morras, R., \& Pöppel, W. G. L. 2005, A\&A, 440, 775

Kallman, T. R., Palmeri, P., Bautista, M. A., Mendoza, C., \& Krolik, J. H. 2004, ApJS, 155, 675

Kaspi, S., Brandt, W. N., George, I. M., Netzer, H., Crenshaw, D. M., Gabel, J. R., Hamann, F. W., Kaiser, M. E., Koratkar, A., Kraemer, S. B., Kriss, G. A., Mathur, S., Mushotzky, R. F., Nandra, K., Peterson, B. M., Shields, J. C., Turner, T. J., \& Zheng, W. 2002, ApJ, 574, 643

Kaspi, S., Brandt, W. N., Netzer, H., George, I. M., Chartas, G., Behar, E., Sambruna, R. M., Garmire, G. P., \& Nousek, J. A. 2001, ApJ, 554, 216

Kaspi, S., Brandt, W. N., Netzer, H., Sambruna, R., Chartas, G., Garmire, G. P., \& Nousek, J. A. 2000, ApJ, 535, L17

Kaspi, S., Maoz, D., Netzer, H., Peterson, B. M., Vestergaard, M., \& Jannuzi, B. T. 2005, ApJ, 629, 61

Kaspi, S., Netzer, H., Chelouche, D., George, I. M., Nandra, K., \& Turner, T. J. 2004, ApJ, 611, 68

King, A. R. \& Pounds, K. A. 2003, MNRAS, 345, 657

Kinkhabwala, A., Sako, M., Behar, E., Kahn, S. M., Paerels, F., Brinkman, A. C., Kaastra, J. S., Gu, M. F., \& Liedahl, D. A. 2002, ApJ, 575, 732

Kraemer, S. B., Ferland, G. J., \& Gabel, J. R. 2004, ApJ, 604, 556

Kraemer, S. B., George, I. M., Crenshaw, D. M., Gabel, J. R., Turner, T. J., Gull, T. R., Hutchings, J. B., Kriss, G. A., Mushotzky, R. F., Netzer, H., Peterson, B. M., \& Behar, E. 2005, ApJ, 633, 693

Krolik, J. H. \& Kriss, G. A. 2001, ApJ, 561, 684

Krongold, Y., Nicastro, F., Brickhouse, N. S., Elvis, M., Liedahl, D. A., \& Mathur, S. 2003, ApJ, 597, 832

Krongold, Y., Nicastro, F., Elvis, M., Brickhouse, N., Binette, L., Mathur, S., \& Jiménez-Bailón, E. 2007, ApJ, 659, 1022

Krongold, Y., Nicastro, F., Elvis, M., Brickhouse, N. S., Mathur, S., \& Zezas, A. 2005, ApJ, 620, 165

Lee, J. C., Ogle, P. M., Canizares, C. R., Marshall, H. L., Schulz, N. S., Morales, R., Fabian, A. C., \& Iwasawa, K. 2001, ApJ, 554, L13

Liedahl, D. A. \& Paerels, F. 1996, ApJ, 468, L33

Lira, P., Arévalo, P., Uttley, P., McHardy, I., \& Breedt, E. 2011, MNRAS, 415, 1290

Lobban, A. P., Reeves, J. N., Miller, L., Turner, T. J., Braito, V., Kraemer, S. B., \& Crenshaw, D. M. 2011, MNRAS, 414, 1965

Longinotti, A. L., Bianchi, S., Ballo, L., de La Calle, I., \& Guainazzi, M. 2009, MNRAS, 394, L1 
Longinotti, A. L., Costantini, E., Petrucci, P. O., Boisson, C., Mouchet, M., Santos-Lleo, M., Matt, G., Ponti, G., \& Gonçalves, A. C. 2010, A\&A, 510, A92

Macchetto, F., Colina, L., Golombek, D., Perryman, M. A. C., \& di Serego Alighieri, S. 1990, ApJ, 356, 389

McKernan, B., Yaqoob, T., George, I. M., \& Turner, T. J. 2003, ApJ, 593, 142

McKernan, B., Yaqoob, T., \& Reynolds, C. S. 2007, MNRAS, 379, 1359

Mineo, T. \& Stewart, G. C. 1993, MNRAS, 262, 817

Monier, E. M., Mathur, S., Wilkes, B., \& Elvis, M. 2001, ApJ, 559, 675

Mor, R., Netzer, H., \& Elitzur, M. 2009, ApJ, 705, 298

Nahar, S. N. \& Pradhan, A. K. 2003, ApJS, 149, 239

Nandra, K., George, I. M., Mushotzky, R. F., Turner, T. J., \& Yaqoob, T. 1997, ApJ, 488, L91

Netzer, H. 1996, ApJ, 473, 781

-. 2004, ApJ, 604, 551

Netzer, H., Kaspi, S., Behar, E., Brandt, W. N., Chelouche, D., George, I. M., Crenshaw, D. M., Gabel, J. R., Hamann, F. W., Kraemer, S. B., Kriss, G. A., Nandra, K., Peterson, B. M., Shields, J. C., \& Turner, T. J. 2003, ApJ, 599, 933

Netzer, H. \& Laor, A. 1993, ApJ, 404, L51

Ogle, P. M., Mason, K. O., Page, M. J., Salvi, N. J., Cordova, F. A., McHardy, I. M., \& Priedhorsky, W. C. 2004, ApJ, 606, 151

Orr, A., Barr, P., Guainazzi, M., Parmar, A. N., \& Young, A. J. 2001, A\&A, 376, 413

Page, K. L., O’Brien, P. T., Reeves, J. N., \& Turner, M. J. L. 2004, MNRAS, 347, 316

Pan, H. C., Stewart, G. C., \& Pounds, K. A. 1990, MNRAS, 242, 177

Papadakis, I. E., Brinkmann, W., Page, M. J., McHardy, I., \& Uttley, P. 2007, A\&A, 461, 931

Patrick, A. R., Reeves, J. N., Porquet, D., Markowitz, A. G., Braito, V., \& Lobban, A. P. 2012, MNRAS, 426,2522

Peterson, B. M., Ferrarese, L., Gilbert, K. M., Kaspi, S., Malkan, M. A., Maoz, D., Merritt, D., Netzer, H., Onken, C. A., Pogge, R. W., Vestergaard, M., \& Wandel, A. 2004, ApJ, 613, 682

Phillips, M. M. 1980, ApJ, 236, L45 
Piconcelli, E., Jimenez-Bailón, E., Guainazzi, M., Schartel, N., Rodríguez-Pascual, P. M., \& Santos-Lleó, M. 2005, A\&A, 432, 15

Ponti, G., Cappi, M., Costantini, E., Bianchi, S., Kaastra, J. S., De Marco, B., Fender, R. P., Petrucci, P.-O., Kriss, G. A., Steenbrugge, K. C., Arav, N., Behar, E., Branduardi-Raymont, G., Dadina, M., Ebrero, J., Lubiński, P., Mehdipour, M., Paltani, S., Pinto, C., \& Tombesi, F. 2013, A\&A, 549, A72

Ponti, G., Cappi, M., Vignali, C., Miniutti, G., Tombesi, F., Dadina, M., Fabian, A. C., Grandi, P., Kaastra, J., Petrucci, P. O., Bianchi, S., Matt, G., Maraschi, L., \& Malaguti, G. 2009, MNRAS, 394, 1487

Porquet, D. \& Dubau, J. 2000, A\&AS, 143, 495

Porquet, D., Dubau, J., \& Grosso, N. 2010, Space Sci. Rev., 157, 103

Porquet, D., Reeves, J. N., O’Brien, P., \& Brinkmann, W. 2004, A\&A, 422, 85

Pounds, K., Reeves, J., O’Brien, P., Page, K., Turner, M., \& Nayakshin, S. 2001, ApJ, 559, 181

Pounds, K. A. \& Reeves, J. N. 2009, MNRAS, 397, 249

Pounds, K. A., Reeves, J. N., King, A. R., \& Page, K. L. 2004a, MNRAS, 350, 10

Pounds, K. A., Reeves, J. N., King, A. R., Page, K. L., O’Brien, P. T., \& Turner, M. J. L. 2003, MNRAS, 345,705

Pounds, K. A., Reeves, J. N., Page, K. L., \& O’Brien, P. T. 2004b, ApJ, 616, 696

Proga, D. \& Kallman, T. R. 2004, ApJ, 616, 688

Raimundo, S. I., Fabian, A. C., Vasudevan, R. V., Gandhi, P., \& Wu, J. 2012, MNRAS, 419, 2529

Ramírez, J. M., Komossa, S., Burwitz, V., \& Mathur, S. 2008, ApJ, 681, 965

Reeves, J. N., Gofford, J., Braito, V., \& Sambruna, R. 2010, ApJ, 725, 803

Reeves, J. N., Nandra, K., George, I. M., Pounds, K. A., Turner, T. J., \& Yaqoob, T. 2004, ApJ, 602, 648

Reeves, J. N., O’Brien, P. T., Braito, V., Behar, E., Miller, L., Turner, T. J., Fabian, A. C., Kaspi, S., Mushotzky, R., \& Ward, M. 2009, ApJ, 701, 493

Reeves, J. N. \& Turner, M. J. L. 2000, MNRAS, 316, 234

Reynolds, C. S. 1997, MNRAS, 286, 513

Ricker, G. R., Clark, G. W., Doxsey, R. E., Dower, R. G., Jernigan, J. G., Delvaille, J. P., MacAlpine, G. M., \& Hjellming, R. M. 1978, Nature, 271, 35

Risaliti, G., Bianchi, S., Matt, G., Baldi, A., Elvis, M., Fabbiano, G., \& Zezas, A. 2005, ApJ, 630, L129 
Sako, M., Kahn, S. M., Behar, E., Kaastra, J. S., Brinkman, A. C., Boller, T., Puchnarewicz, E. M., Starling, R., Liedahl, D. A., Clavel, J., \& Santos-Lleo, M. 2001, A\&A, 365, L168

Schmidt, E. W., Schippers, S., Müller, A., Lestinsky, M., Sprenger, F., Grieser, M., Repnow, R., Wolf, A., Brandau, C., Lukić, D., Schnell, M., \& Savin, D. W. 2006, ApJ, 641, L157

Scott, A. E., Stewart, G. C., Mateos, S., Alexander, D. M., Hutton, S., \& Ward, M. J. 2011, MNRAS, 417, 992

Scott, J. E., Kriss, G. A., Lee, J. C., Arav, N., Ogle, P., Roraback, K., Weaver, K., Alexander, T., Brotherton, M., Green, R. F., Hutchings, J., Kaiser, M. E., Marshall, H., Oegerle, W., \& Zheng, W. 2004, ApJS, 152,1

Shu, X. W., Yaqoob, T., \& Wang, J. X. 2010, ApJS, 187, 581

Smith, R. A. N., Page, M. J., \& Branduardi-Raymont, G. 2007, A\&A, 461, 135

Steenbrugge, K. C., Fenovčík, M., Kaastra, J. S., Costantini, E., \& Verbunt, F. 2009, A\&A, 496, 107

Steenbrugge, K. C., Kaastra, J. S., Crenshaw, D. M., Kraemer, S. B., Arav, N., George, I. M., Liedahl, D. A., van der Meer, R. L. J., Paerels, F. B. S., Turner, T. J., \& Yaqoob, T. 2005a, A\&A, 434, 569

Steenbrugge, K. C., Kaastra, J. S., Sako, M., Branduardi-Raymont, G., Behar, E., Paerels, F. B. S., Blustin, A. J., \& Kahn, S. M. 2005b, A\&A, 432, 453

Tarter, C. B., Tucker, W. H., \& Salpeter, E. E. 1969, ApJ, 156, 943

Tatum, M. M., Turner, T. J., Miller, L., \& Reeves, J. N. 2013, ApJ, 762, 80

Terashima, Y., Gallo, L. C., Inoue, H., Markowitz, A. G., Reeves, J. N., Anabuki, N., Fabian, A. C., Griffiths, R. E., Hayashida, K., Itoh, T., Kokubun, N., Kubota, A., Miniutti, G., Takahashi, T., Yamauchi, M., \& Yonetoku, D. 2009, PASJ, 61, 299

Tombesi, F., Cappi, M., Reeves, J. N., \& Braito, V. 2012, MNRAS, 422, L1

Tombesi, F., Cappi, M., Reeves, J. N., Nemmen, R. S., Braito, V., Gaspari, M., \& Reynolds, C. S. 2013, MNRAS, 430, 1102

Tombesi, F., Cappi, M., Reeves, J. N., Palumbo, G. G. C., Braito, V., \& Dadina, M. 2011, ApJ, 742, 44

Tombesi, F., Cappi, M., Reeves, J. N., Palumbo, G. G. C., Yaqoob, T., Braito, V., \& Dadina, M. 2010a, A\&A, 521, A57

Tombesi, F., Sambruna, R. M., Reeves, J. N., Braito, V., Ballo, L., Gofford, J., Cappi, M., \& Mushotzky, R. F. 2010b, ApJ, 719, 700

Turner, A. K., Fabian, A. C., Lee, J. C., \& Vaughan, S. 2004, MNRAS, 353, 319 
Turner, T. J., Kraemer, S. B., George, I. M., Reeves, J. N., \& Bottorff, M. C. 2005, ApJ, 618, 155

Turner, T. J., Reeves, J. N., Kraemer, S. B., \& Miller, L. 2008, A\&A, 483, 161

Urry, C. M. \& Padovani, P. 1995, PASP, 107, 803

Vasudevan, R. V. \& Fabian, A. C. 2009, MNRAS, 392, 1124

Weisskopf, M. C., Hester, J. J., Tennant, A. F., Elsner, R. F., Schulz, N. S., Marshall, H. L., Karovska, M., Nichols, J. S., Swartz, D. A., Kolodziejczak, J. J., \& O’Dell, S. L. 2000, ApJ, 536, L81

Wilms, J., Allen, A., \& McCray, R. 2000, ApJ, 542, 914

Yaqoob, T., McKernan, B., Kraemer, S. B., Crenshaw, D. M., Gabel, J. R., George, I. M., \& Turner, T. J. 2003, ApJ, 582, 105

Zhang, S. N., Ji, L., Marshall, H. L., Longinotti, A. L., Evans, D., \& Gu, Q. S. 2011, MNRAS, 410, 2274 


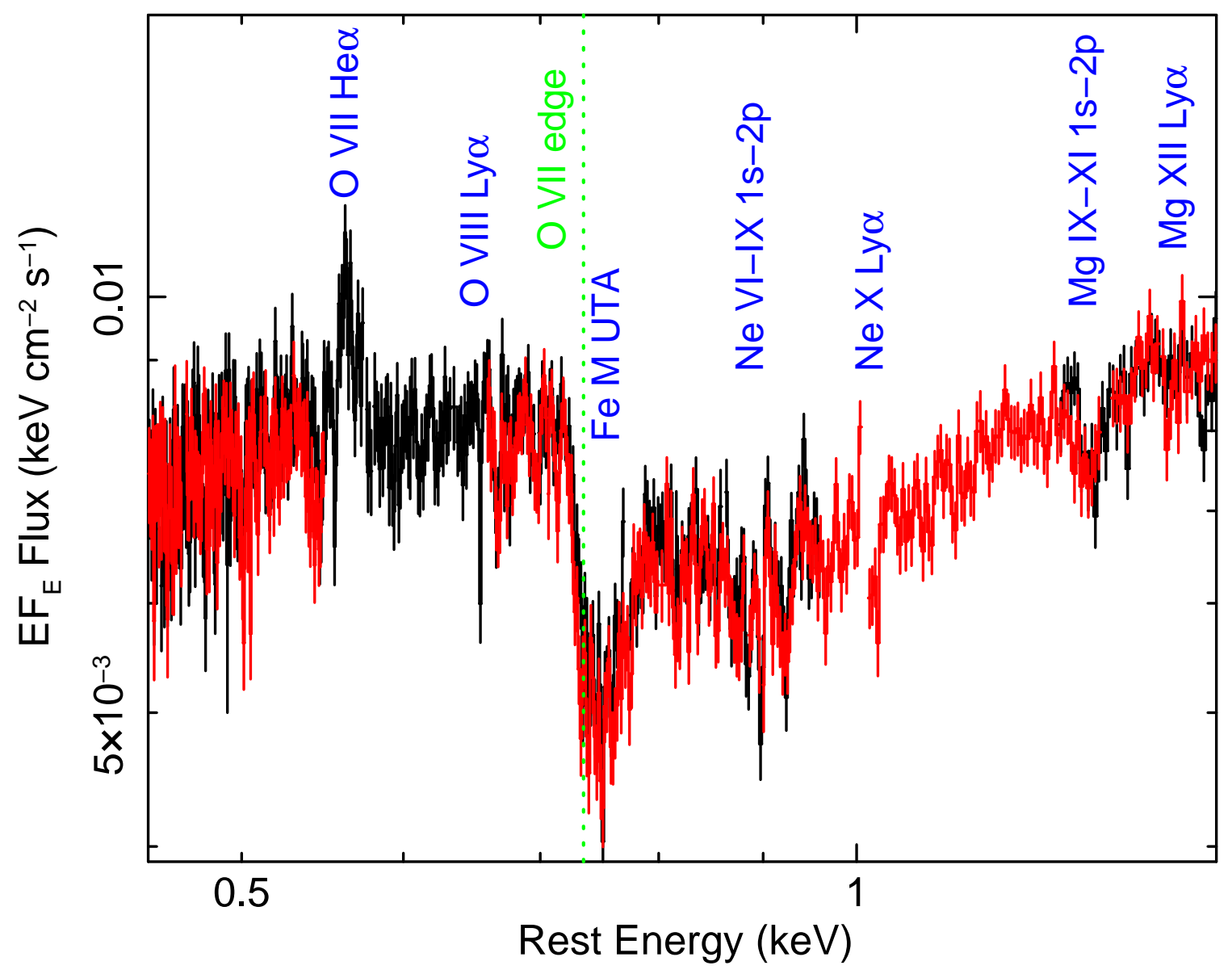

Fig. 1.- Fluxed 2011 XMM-Newton RGS spectra of MR 2251-178 between 0.4-1.5 keV. RGS 1 is shown in black, RGS 2 in red. The spectrum shows a clear imprint of a warm absorber, with the main features in the spectrum labelled. The absorption due to the UTA of M-shell iron is particularly prominent above 0.7 $\mathrm{keV}$, as well as absorption due to $\mathrm{Ne}$ (and iron L-shell) between $0.9-1.0 \mathrm{keV}$, as well as an absorption trough due to $\mathrm{Mg}$ near $1.3 \mathrm{keV}$. Note the strong O VII emission at $0.56-0.57 \mathrm{keV}$. Energy is plotted in the quasar rest frame. 


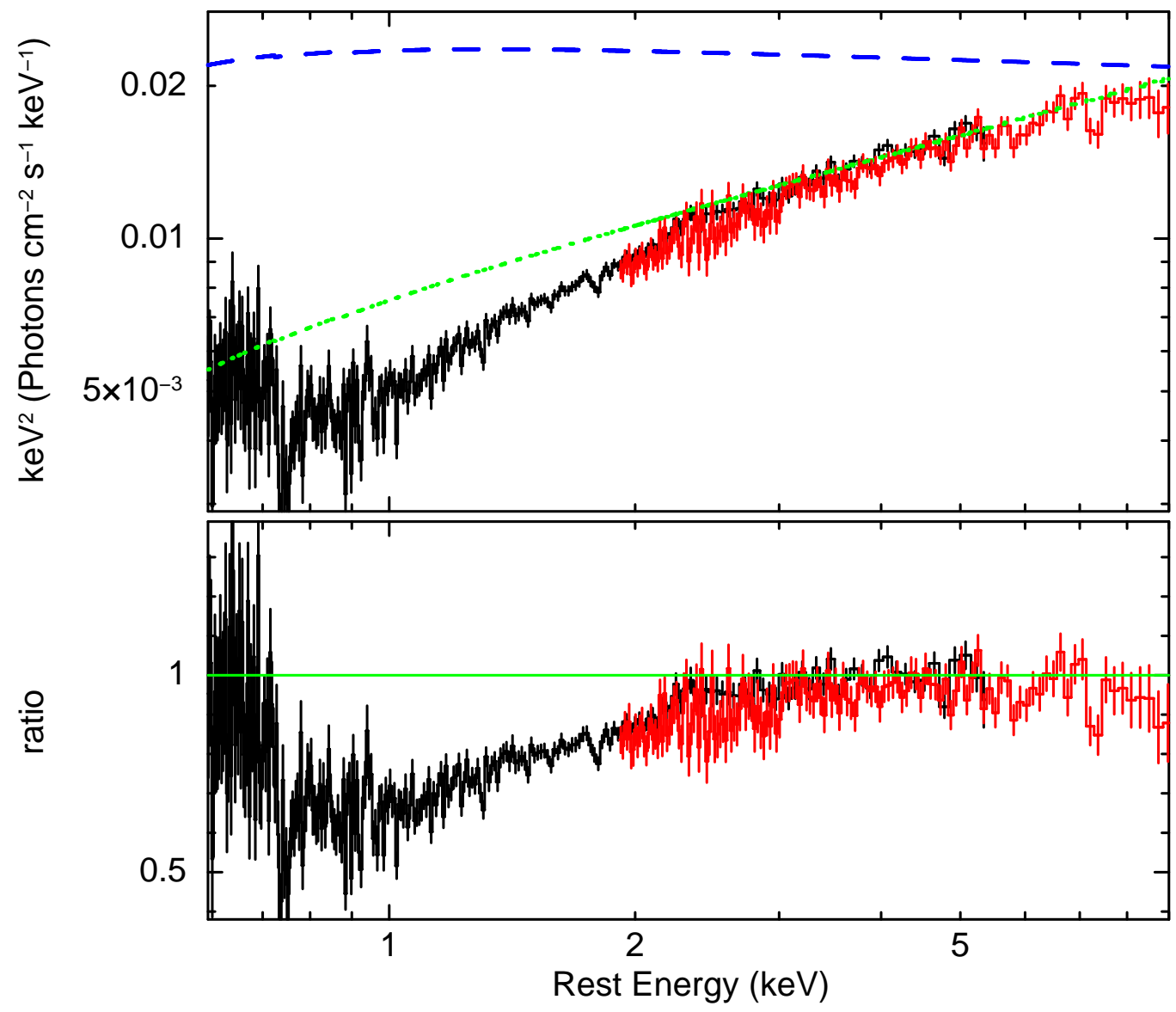

Fig. 2.- Fluxed 2011 Chandra HETG spectra of MR 2251-178, MEG is shown in black, HEG is red. The top panel shows the spectra, while the dotted green line is a representative power-law continuum with $\Gamma=1.6$. The upper dashed blue line shows the actual intrinsic level of the continuum, once the absorption is modeled. The lower panel shows data/model ratio to the $\Gamma=1.6$ power-law, the downwards continuum curvature due to the warm absorber is clearly present. Note that data are binned at $4 \times$ the HWHM resolution for clarity, while the HEG spectrum is only plotted above $2 \mathrm{keV}$. 


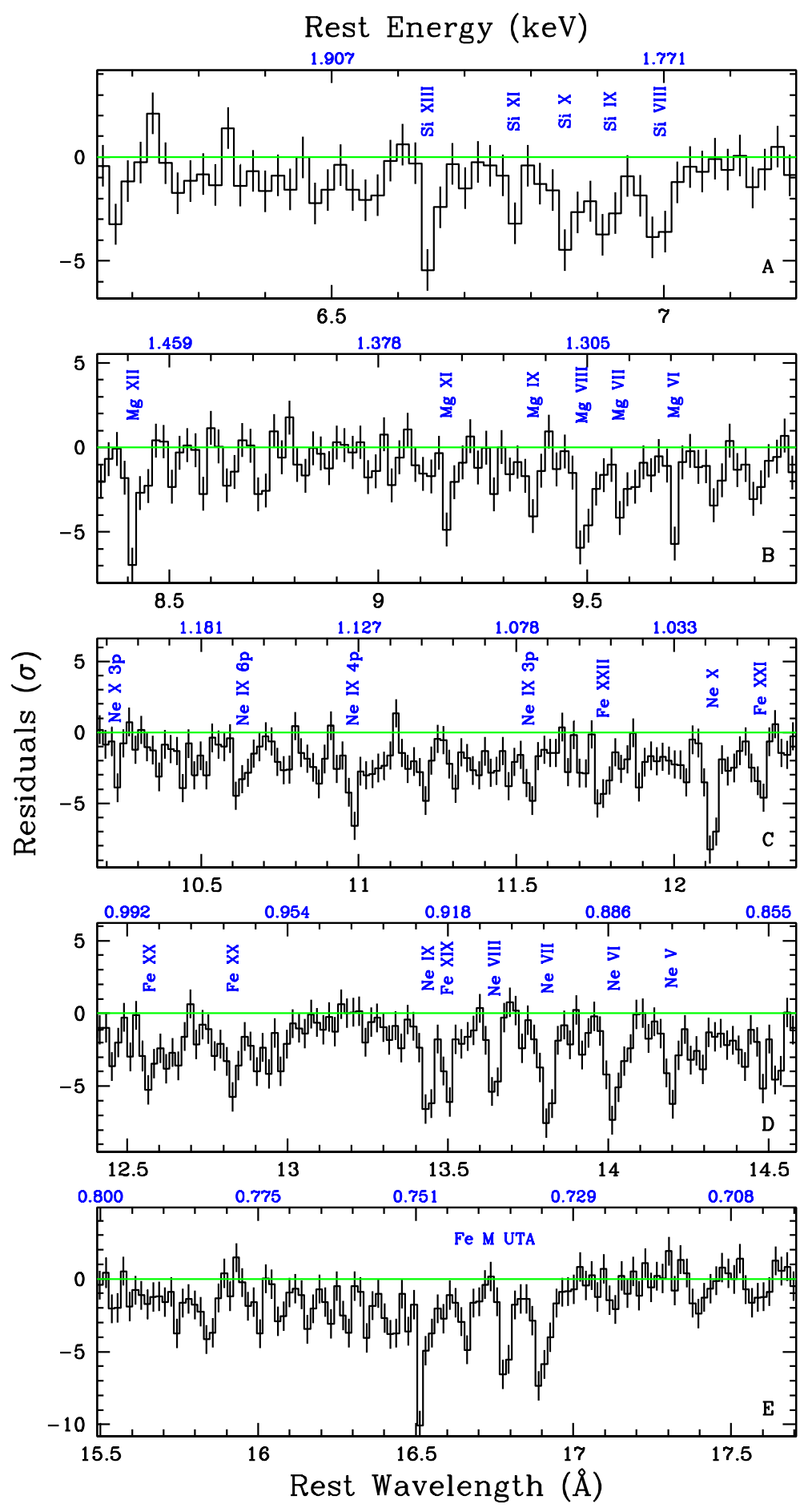

Fig. 3.- 2011 Chandra MEG spectrum of MR 2251-178, showing the wealth of absorption lines below $2 \mathrm{keV}$. The data are shown as residuals (in $\sigma$ ) against the baseline continuum and are plotted in the quasar rest frame in wavelength (with energy marked along the upper axis). Panel (a) shows the Si K band, including inner shell absorption from Si VIII-XIII; (b) the Mg K band, including inner shell absorption from Mg VI-XI; (c) absorption from Ne IX, Ne X and L-shell Fe; (d) inner shell absorption due to Neon ions from Ne V-Ne IX and (e) the iron M-shell UTA band. 


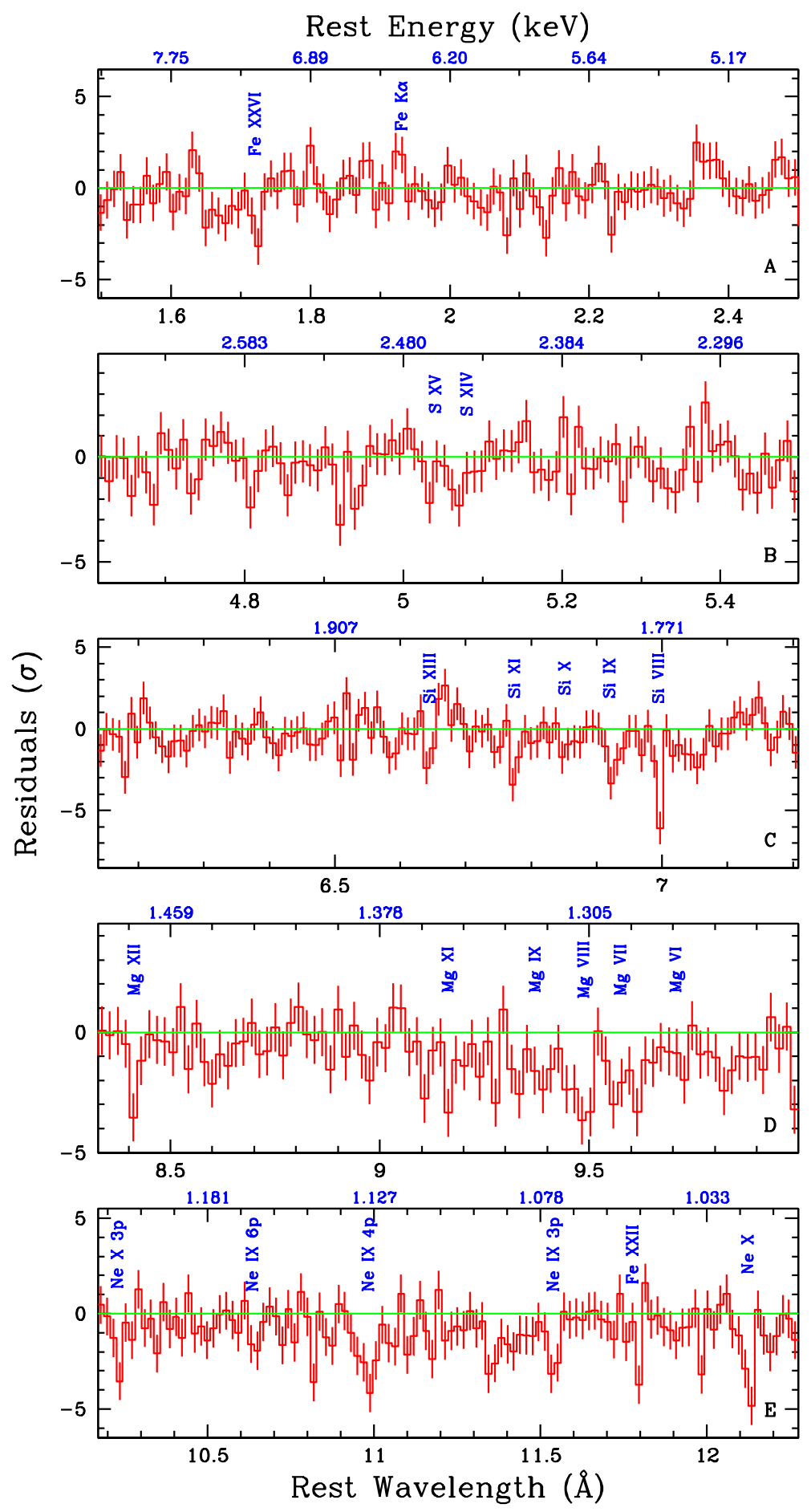

Fig. 4.- As per Figure 3, but showing the Chandra HEG spectrum of MR 2251-178, Panels (c), (d) and (e) show the absorption present in the $\mathrm{Si}, \mathrm{Mg}$ and $\mathrm{Ne}$ bands respectively. Panels (a) and (b) also show the spectrum in the Fe and $\mathrm{S} \mathrm{K}$-shell bands. 

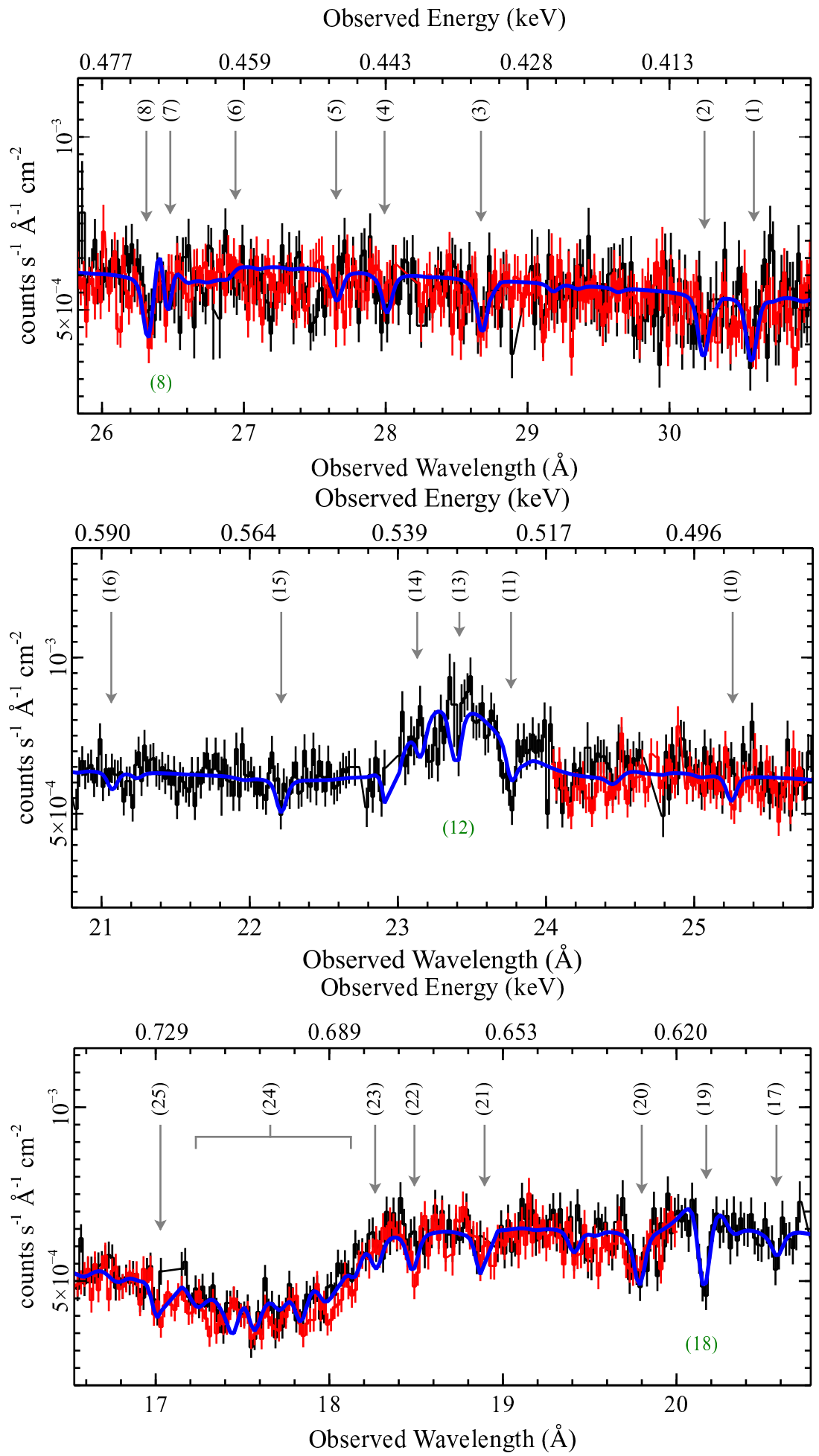

Fig. 5. - Enlarged view of the XMM-Newton/RGS data (RGS1: black, RGS2: red), showing the count rate spectra normalized to the instrument effective area. The best-fit model for the soft X-ray absorber, comprising three absorption components, is shown by the blue line. There are a plethora of ionized lines present originating from $\mathrm{C}, \mathrm{N}, \mathrm{O}$ and the Fe M-shell. There is also some complex interplay between the absorption and underlying emission components, which are further discussed in the text. The likely identification of the numbered lines are presented in Table 3 Green numbers denote the position of emission components. 
Observed Energy (keV)
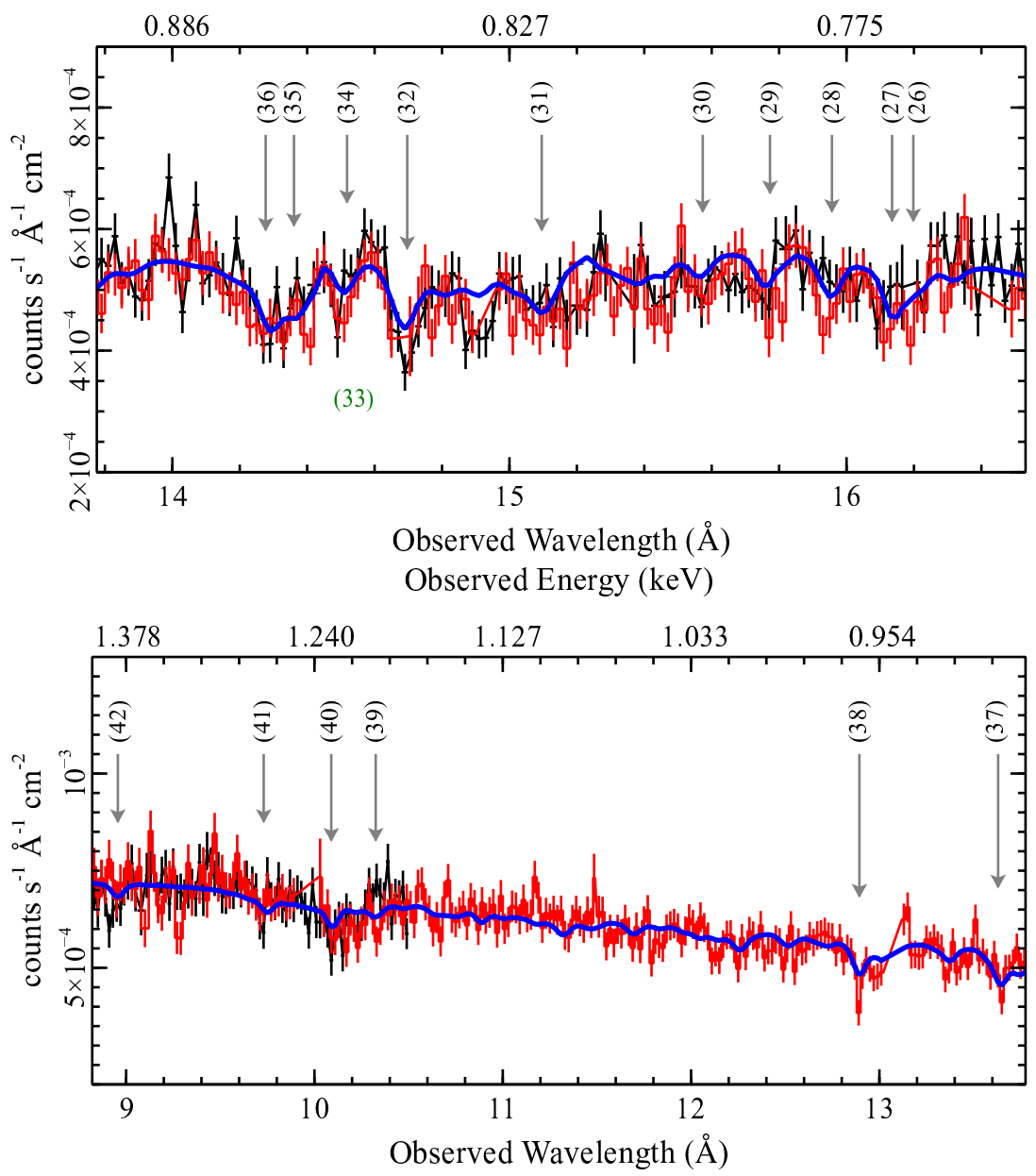

Fig. 6.- Enlarged view of the XMM-Newton/RGS data showing the $\mathrm{Ne}$ and $\mathrm{Mg}$ energy regimes. The blue line again shows the best-fit absorption model. Both $\mathrm{Ne}$ and $\mathrm{Mg}$ have a number of inner-shell lines (i.e., the B-, Be-, Li-like charge states) present in the spectrum. As in the Figure 5, the likely identification of the numbered lines are presented in Table 3 . 

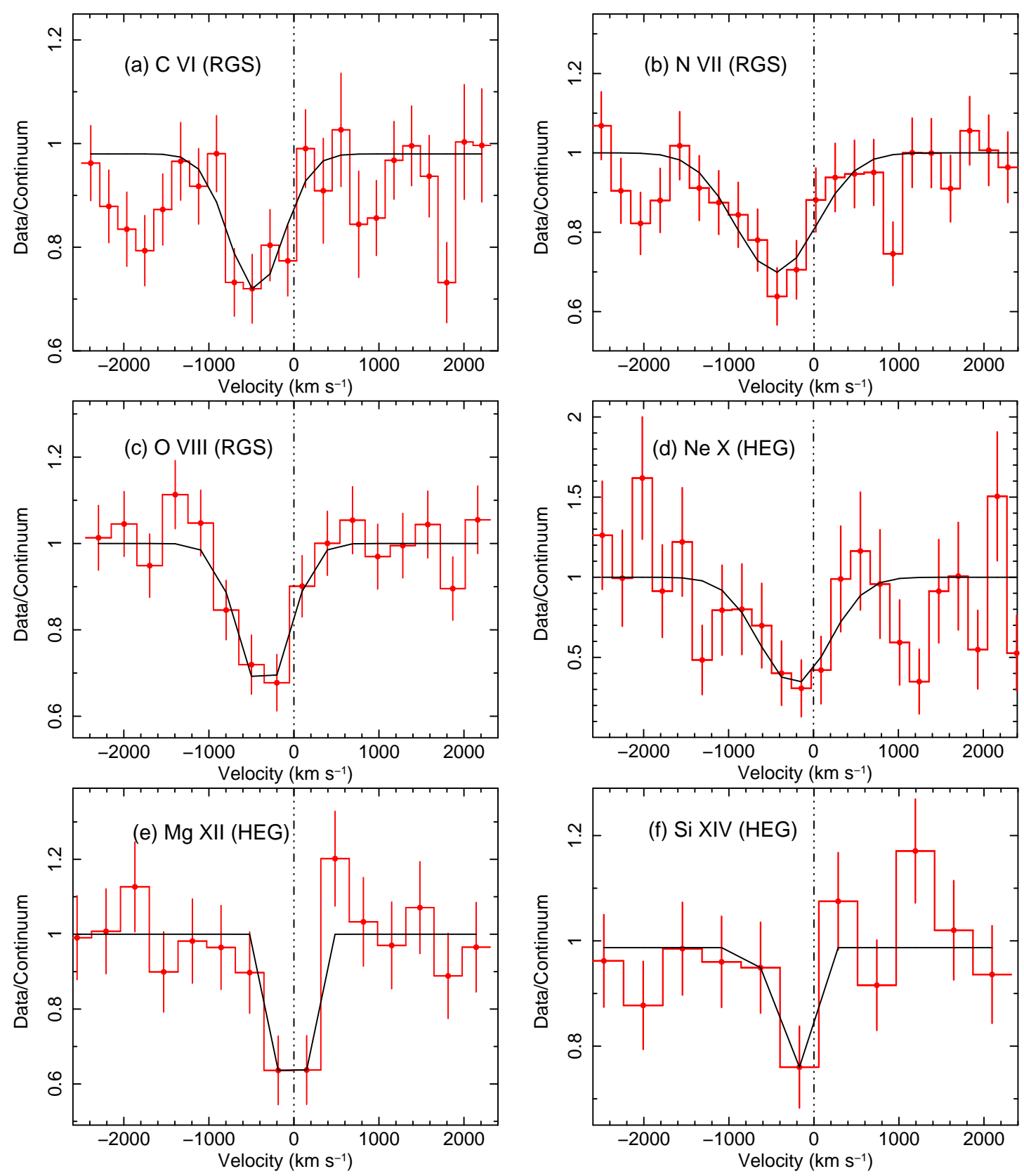

Fig. 7.- Velocity profiles of the main H-like lines, as measured by XMM-Newton RGS (for C VI, N VII, $\mathrm{O}$ VIII) and Chandra HEG (for Ne X, Mg XII, Si XIV), see Section 3.4 for details. The data points show the data divided by continuum model for each line and negative velocities correspond to blue-shifts. The solid line indicates the simple single Gaussian absorption profile fitted to each line profile. In the case of the $\mathrm{C}, \mathrm{N}$, $\mathrm{O}$ (and to a lesser extent $\mathrm{Ne}$ ) lines, a clear blue-shift of the Gaussian centroid is observed, while the higher energy $\mathrm{Mg}$ and Si lines do not require any net blue-shift and appear unresolved. The subsequent best-fit values of the Gaussian profiles are reported in Table 4 

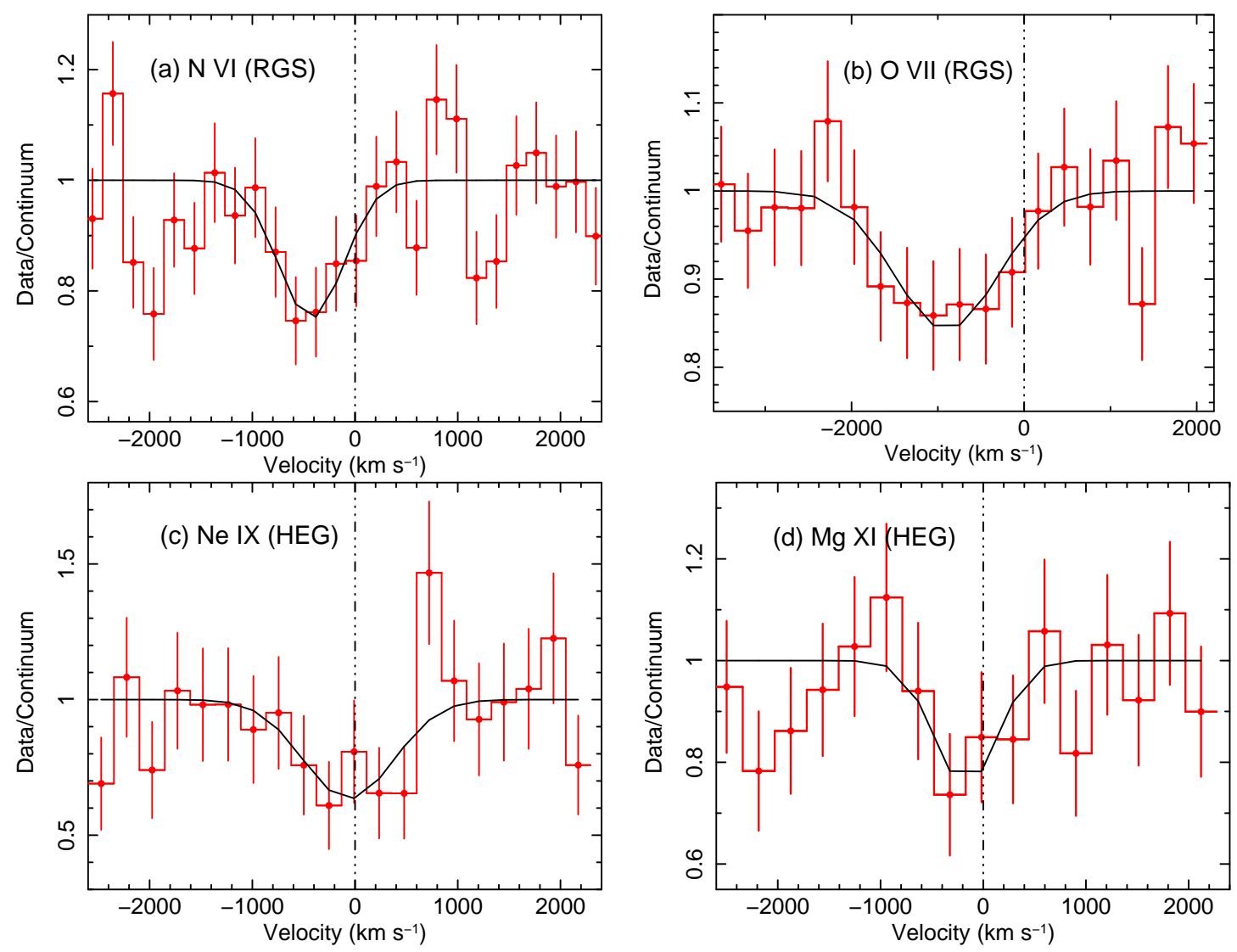

Fig. 8.- As per Figure7, except the velocity profiles correspond to the He-like lines of N VI, O VII (RGS) and Ne IX, Mg XI (HEG). 

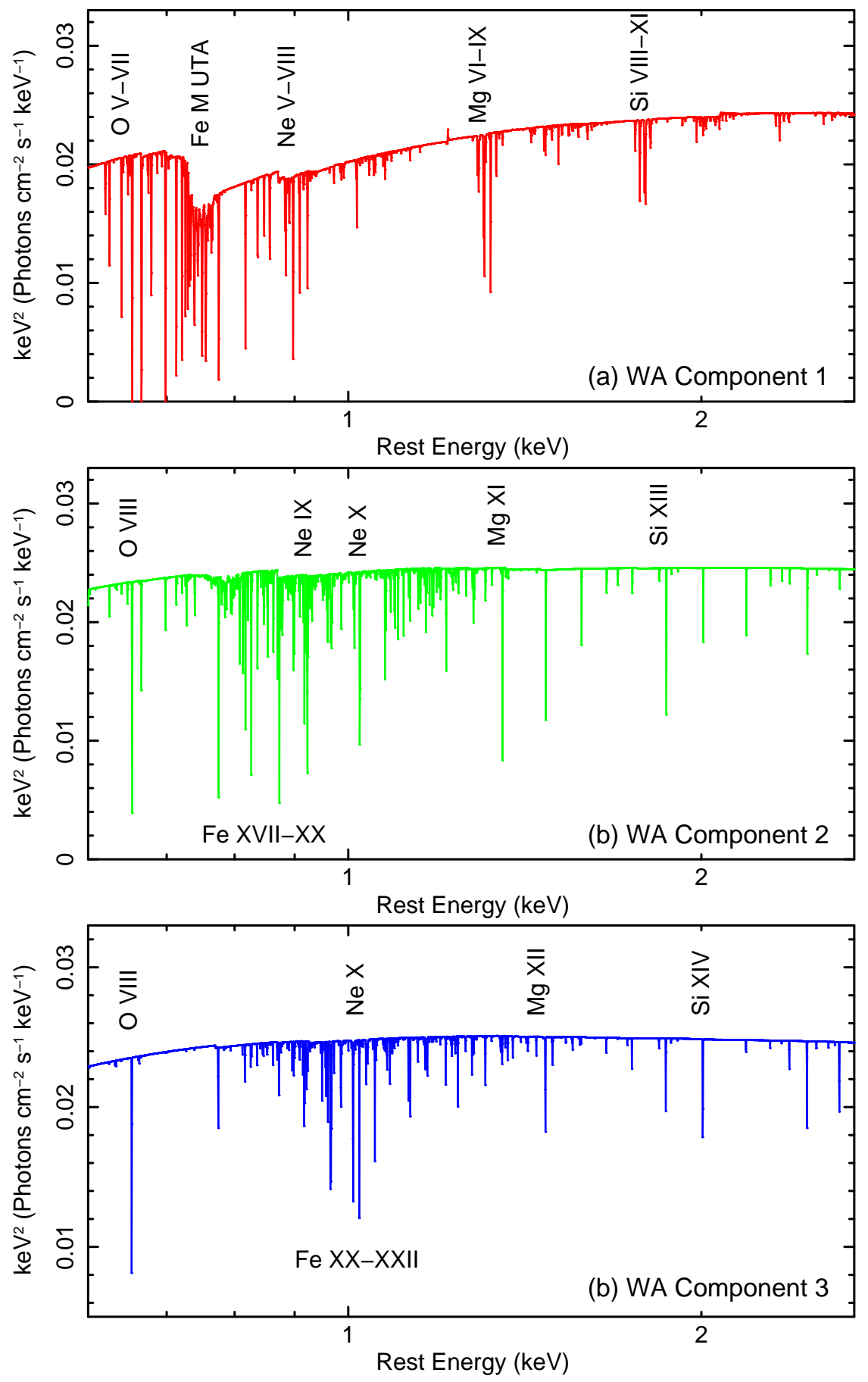

Fig. 9.- Contribution of respective warm absorption components towards the X-ray spectrum. The low ionization component 1 (panel a) carries the largest opacity with absorption due to inner shell $\mathrm{O}, \mathrm{Ne}, \mathrm{Mg}, \mathrm{Si}$ and M-shell Fe; component 2 (panel b) contains absorption due to He-like ions and moderately ionized Fe and component 3 (panel c) contributes absorption due to H-like ions and highly ionized Fe. 

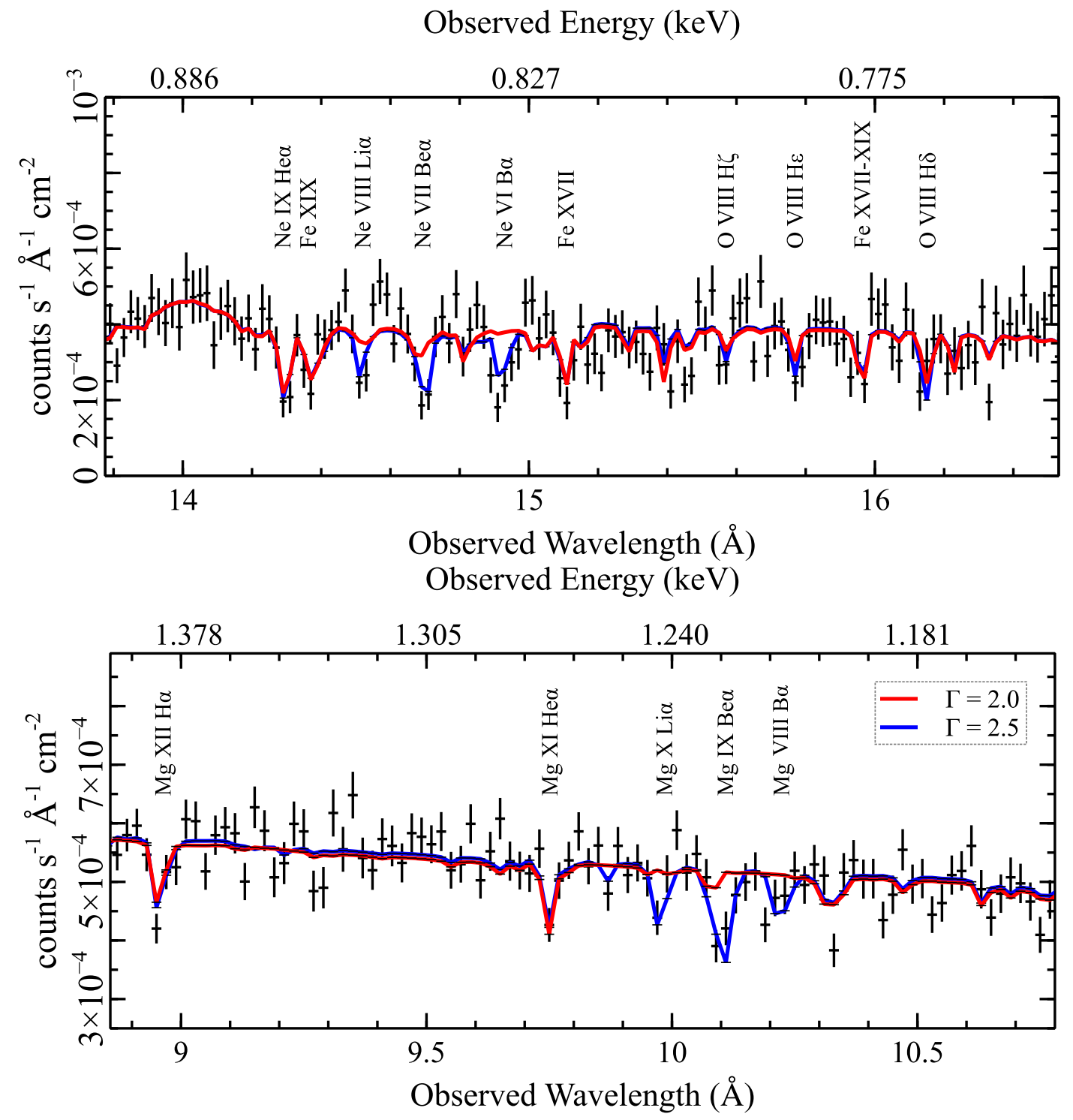

Fig. 10.- Enlarged portions of the 2011 Chandra/HETG observation of MR 2251-178, focusing on the $\mathrm{Ne}$ and $\mathrm{Mg}$ energy bands. The HETG data give a much clearer view of the inner-shell $\mathrm{Ne}$ and $\mathrm{Mg}$ lines than was possible with the XMM-Newton/RGS. Both elements have lines due to their B-, Be- and Li-like charge states. The solid lines correspond to the fit that is obtained when the low ionization xstar absorber (component 1, Table 5) has an input photon continuum of $\Gamma_{\text {input }}=2.0$ (red) and $\Gamma_{\text {input }}=2.5$ (blue). Importantly, the inner-shell lines cannot be accounted for without an intrinsically soft X-ray continuum, which in turn provides evidence for a partially-covered X-ray spectrum. See text for further details. 

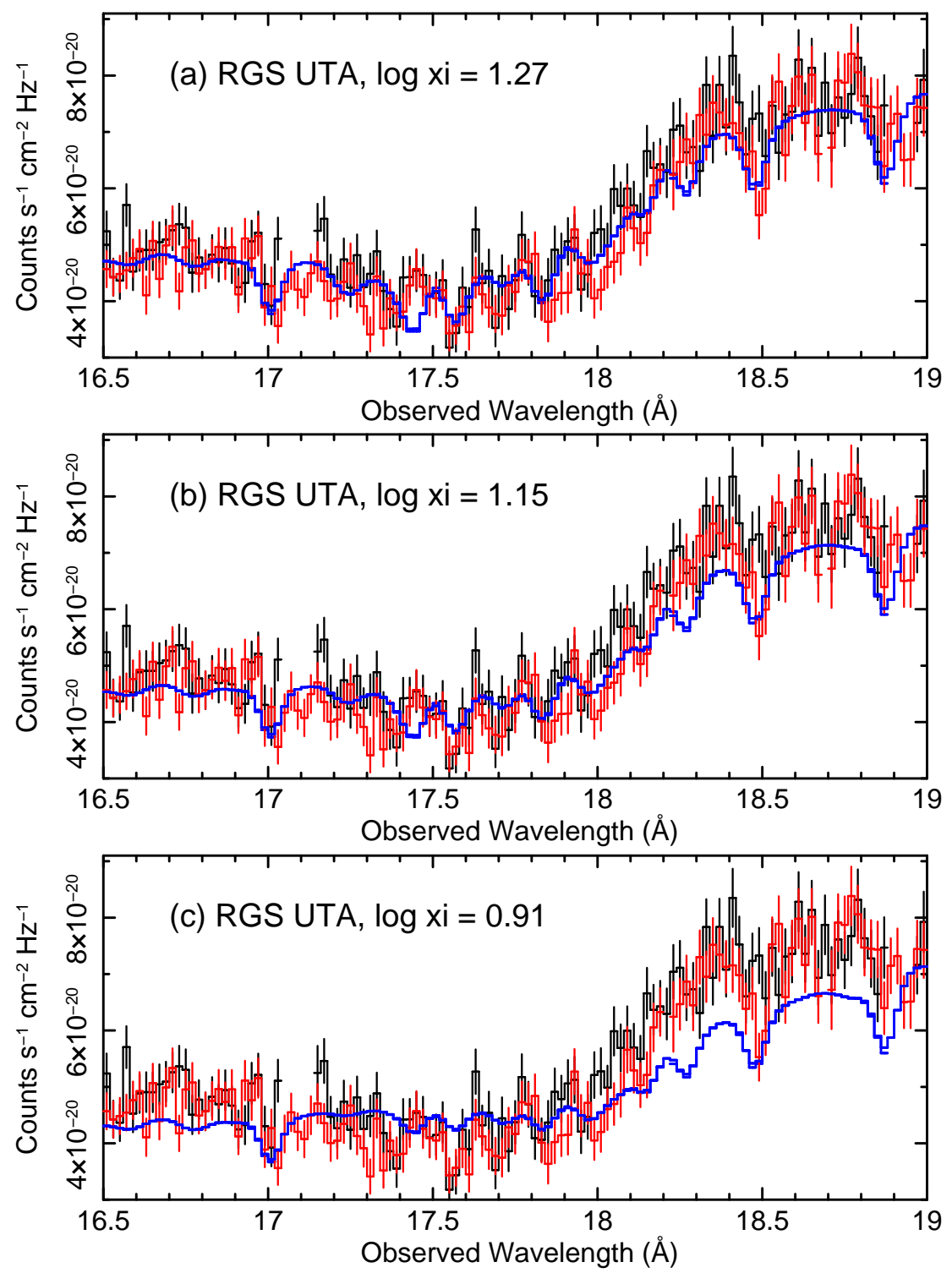

Fig. 11.-Zoom-in of the 2011 RGS spectrum in the region of the iron M-shell UTA, showing the effect of the change in the ionization state of component 1 in the warm absorber model (solid line). Panel (a) shows the best fit case to the RGS, whereby the ionization parameter of component 1 is $\log \left(\xi / \mathrm{erg} \mathrm{cm} \mathrm{s}^{-1}\right)=1.27$. Panel (b) shows the model fitted when the ionization is lowered to $\log \left(\xi / \mathrm{erg} \mathrm{cm} \mathrm{s}^{-1}\right)=1.15$, as found in the 2011 Chandra HETG spectral fits. In panel (c) the ionization parameter is $\log \left(\xi / \mathrm{erg} \mathrm{cm} \mathrm{s}^{-1}\right)=0.91$, as found in the spectral fits to the 2002 Chandra HETG data. Thus the fits to the UTA region are sensitive to the ionization parameter in the xstar absorber model and models with a substantially lower ionization, as found in the Chandra datasets, can be ruled out by the RGS data. 


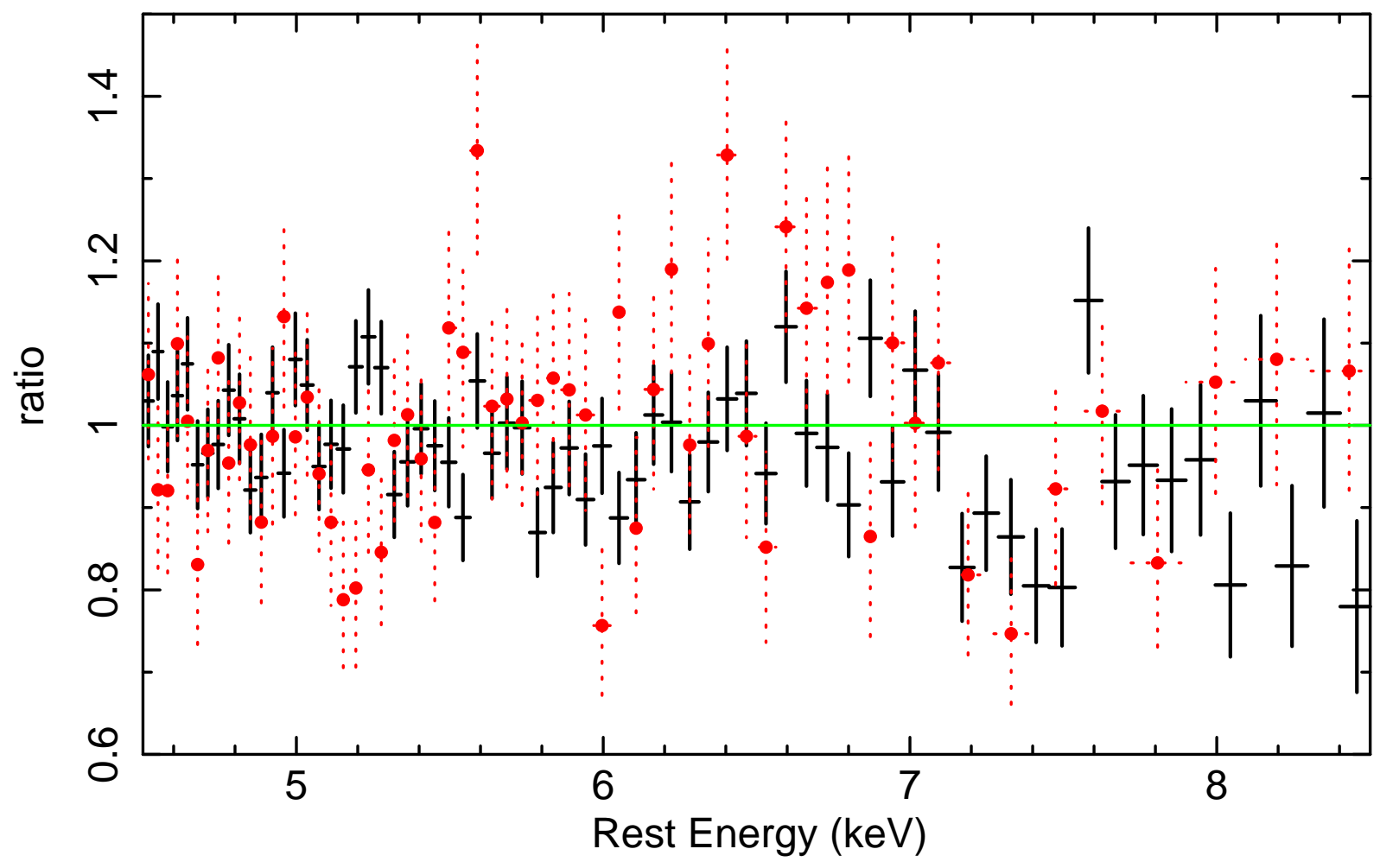

Fig. 12.- Data/model ratio residuals to the HEG spectrum of MR 2251-178, in the iron K band, to the best fit continuum model. The 2011 data are shown as black crosses, the 2002 HEG data shown in red circles (with dashed errors). The datasets have also been binned to have a minimum of 20 counts per bin, in addition to the instrument resolution binning. Energy is plotted in the quasar rest frame at $z=0.064$. Both datasets appear to show a weak, but statistically required, absorption feature near $7.3 \mathrm{keV}$, which if identified with $\mathrm{H}$-like iron would require a blueshift of $\sim-15000 \mathrm{~km} \mathrm{~s}^{-1}$. Note the lack of a strong $\mathrm{K} \alpha$ emission line at $6.4 \mathrm{keV}$. 


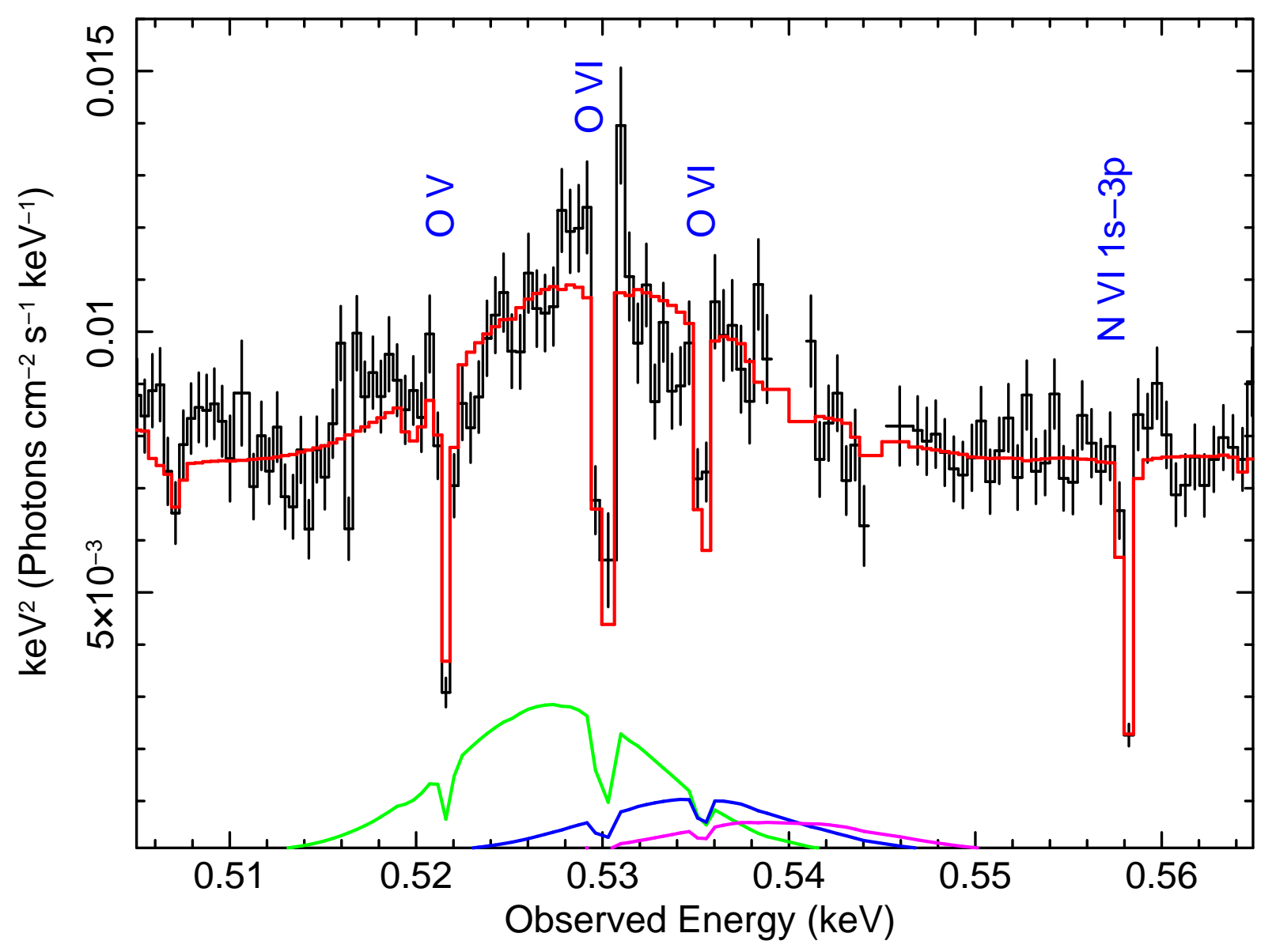

Fig. 13.- Broad O VII emission line complex as observed by RGS 1, plotted against observed frame energy. Black crosses show the data, while the solid (red) line is the best fit model. The line profile has been fitted by a blend of forbidden, intercombination and resonance emission components of equal velocity width (FWHM $7300 \mathrm{~km} \mathrm{~s}^{-1}$ ), as shown by the solid lines below from left to right (green, blue and magenta respectively). The forbidden line dominates the profile, implying densities of $n_{e}<10^{11} \mathrm{~cm}^{-2}$. Note that narrow absorption lines of $\mathrm{O}$ V-VI are superimposed upon the broad emission profile. 
Table 1. Summary of MR 2251-178 Observations

\begin{tabular}{cccccc}
\hline \hline Mission & Obsid & Start Date/Time $^{a}$ & Inst & Exposure (ks) & Net Rate s $^{-1}$ \\
\hline XMM-Newton & 0670120201 & $2011-11-11$ 07:58:17 & RGS 1 & 133.1 & $0.518 \pm 0.002$ \\
& - & - & RGS 2 & - & $0.562 \pm 0.002$ \\
& 0670120301 & $2011-11-13$ 18:50:38 & RGS 1 & 127.8 & $0.506 \pm 0.002$ \\
& - & - & RGS 2 & - & $0.544 \pm 0.002$ \\
& 0670120401 & $2011-11-1518: 42: 16$ & RGS 1 & 128.0 & $0.464 \pm 0.002$ \\
& - & - & RGS 2 & - & $0.501 \pm 0.002$ \\
& Total & - & RGS 1 & $389.1^{b}$ & $0.496 \pm 0.001$ \\
& & - & RGS 2 & $389.1^{b}$ & $0.535 \pm 0.001$ \\
\hline Chandra & 2977 & $2002-09-1100: 52: 46$ & MEG & 146.3 & $0.317 \pm 0.001$ \\
& - & - & HEG & - & $0.164 \pm 0.001$ \\
\hline Chandra & 12828 & $2011-09-2620: 34: 38$ & MEG & 163.1 & \\
& - & - & HEG & - & \\
& 12829 & $2011-09-2907: 03: 04$ & MEG & 187.6 & \\
& - & - & HEG & - & \\
& 12830 & $2011-10-0122: 53: 18$ & MEG & 48.6 & \\
& - & - & HEG & - & \\
& Total & - & MEG & 392.9 & $0.485 \pm 0.001$ \\
& - & - & HEG & - & $0.245 \pm 0.001$ \\
\hline
\end{tabular}

${ }^{\mathrm{a}}$ Observation Start/End times are in UT.

${ }^{\mathrm{b}} \mathrm{Net}$ exposure time, after screening and deadtime correction, in ks. 
Table 2. Soft X-ray absorption lines in 2011 Chandra HETG Spectrum

\begin{tabular}{|c|c|c|c|c|}
\hline $\mathrm{ID}^{a}$ & $\mathrm{E}_{\text {atomic }}^{b}$ & $\mathrm{E}_{\text {quasar }}^{c}$ & $\mathrm{EW}^{c}$ & $\Delta C^{d}$ \\
\hline O VIII & $653.5[18.972]$ & $654.4_{-0.3}^{+0.1}[18.946]$ & $-0.8 \pm 0.3$ & 9.8 \\
\hline O VIII & - & $733.3_{-0.9}^{+0.5}[16.908]$ & $-1.6 \pm 0.5$ & 20.4 \\
\hline Fe IX-X $2 p-3 d$ & - & $750.4 \pm 1.2[16.522]$ & $-1.5 \pm 0.6$ & 18.0 \\
\hline $\mathrm{Ne} V$ & 871.4 [14.228], 873.7 [14.191] & $873.3 \pm 0.5[14.197]$ & $-1.2 \pm 0.4$ & 15.6 \\
\hline $\mathrm{Ne}$ VI & 885.0 [14.010], 883.3 [14.036] & $884.5 \pm 0.5[14.017]$ & $-1.7 \pm 0.4$ & 27.3 \\
\hline $\mathrm{Ne}$ VII & $898.2[13.804]$ & $897.7 \pm 0.4[13.811]$ & $-1.6 \pm 0.5$ & 29.9 \\
\hline Ne VIII & $909.2[13.637]$ & $908.6 \pm 0.3[13.646]$ & $-1.0 \pm 0.3$ & 12.1 \\
\hline Fe XIX $2 p-3 d$ & $918.0[13.506]$ & $918.6_{-0.4}^{+0.2}[13.497]$ & $-1.0 \pm 0.4$ & 15.2 \\
\hline $\mathrm{Ne}$ IX & $922.0[13.447]$ & $922.6 \pm 0.4[13.439]$ & $-1.5 \pm 0.5$ & 25.7 \\
\hline $\mathrm{Fe} \times \mathrm{X} 2 p-3 d$ & $967.3[12.818]$ & $966.4 \pm 0.7[12.829]$ & $-1.3 \pm 0.5$ & 17.2 \\
\hline $\mathrm{Fe} \times \mathrm{XX} 2 p-3 d$ & $987.8[12.552]$ & $986.4 \pm 0.8[12.569]$ & $-1.2 \pm 0.4$ & 14.7 \\
\hline Fe XXI $2 p-3 d$ & $1000.9[12.387]$ & $1010.1 \pm 0.8[12.274]$ & $-0.9 \pm 0.4$ & 9.8 \\
\hline $\operatorname{Ne} \mathrm{X}$ & 1021.5 [12.137], 1022.0 [12.132] & $1022.8 \pm 0.4[12.122]$ & $-1.8 \pm 0.3$ & 56.6 \\
\hline Fe XXII $2 p-3 d$ & $1053.6[11.768]$ & $1052.9 \pm 1.0[11.766]$ & $-1.0 \pm 0.4$ & 13.6 \\
\hline Ne IX $1 s-3 p$ & $1073.8[11.546]$ & $1074.1 \pm 0.7[11.543]$ & $-1.0 \pm 0.3$ & 18.5 \\
\hline $\mathrm{Ne}$ IX $1 s-4 p$ & $1127.1[11.000]$ & $1128.6 \pm 0.6[10.986]$ & $-1.2 \pm 0.3$ & 36.3 \\
\hline Ne IX $1 s-6 p$ & $1165.0[10.642]$ & $1165.5 \pm 0.7[10.638]$ & $-0.7 \pm 0.3$ & 11.2 \\
\hline Ne $\mathrm{X} 1 s-3 p$ & 1210.9 [10.239] & $1211.7 \pm 0.5[10.232]$ & $-0.6 \pm 0.3$ & 11.6 \\
\hline $\mathrm{Mg}$ VI & $1276.8[9.711]$ & $1276.9 \pm 0.9[9.710]$ & $-0.7 \pm 0.3$ & 12.6 \\
\hline Mg VII & $1291.6[9.599]$ & $1294.5 \pm 1.3[9.578]$ & $-0.7 \pm 0.3$ & 10.8 \\
\hline Mg VIII & $1306.4[9.491],(1304.2$ [9.507]) & $1306.4 \pm 0.7[9.491]$ & $-1.4 \pm 0.3$ & 41.8 \\
\hline Mg IX & $1323.1[9.371]$ & $1322.6 \pm 1.1[9.374]$ & $-0.6 \pm 0.3$ & 9.7 \\
\hline $\operatorname{Mg} X I$ & $1353.1[9.163]$ & $1352.7 \pm 0.8[9.166]$ & $-0.8 \pm 0.3$ & 18.2 \\
\hline Mg XII & $1472.6[8.419], 1471.7[8.425]$ & $1473.0 \pm 0.5[8.417]$ & $-1.2 \pm 0.2$ & 47.3 \\
\hline $\operatorname{Mg}$ XI $1 s-3 p$ & $1579.3[7.851]$ & $1581.3 \pm 0.9[7.841]$ & $-0.6 \pm 0.3$ & 12.6 \\
\hline Si VIII & $1772.8[6.994]$ & $1772.6 \pm 0.6[6.994]$ & $-1.3 \pm 0.3$ & 38.8 \\
\hline Si IX & $1792.2[6.918],(1788.2[6.933])$ & $1791.9_{-1.2}^{+0.9}[6.919]$ & $-0.9 \pm 0.3$ & 19.2 \\
\hline $\mathrm{Six}$ & 1810.3 [6.849], 1807.3 [6.860] & $1809.6_{-2.2}^{+1.4}[6.851]$ & $-0.8 \pm 0.3$ & 15.5 \\
\hline Si XI & $1830.6[6.773]$ & $1830.2 \pm 1.1[6.774]$ & $-0.7 \pm 0.3$ & 14.1 \\
\hline Si XIII & $1866.4[6.643]$ & $1866.0 \pm 0.9[6.644]$ & $-1.0 \pm 0.3$ & 23.5 \\
\hline Si XIV & $2006.1[6.180],(2004.8$ [6.184]) & $2007.0 \pm 1.2[6.178]$ & $-0.8 \pm 0.4$ & 12.3 \\
\hline
\end{tabular}

${ }^{\text {a }}$ Line identification. Lines correspond to the $1 s-2 p$ transition unless stated.

${ }^{\mathrm{b}}$ Known atomic line energy in eV. Values are from www.nist.gov, Behar et al. (2001) for Fe M-shell UTA and Behar \& Netzer (2002) for inner shell $\mathrm{Ne}, \mathrm{Mg}$, Si. The corresponding wavelength in $\AA$ is given within brackets. 
${ }^{\mathrm{c}}$ Measured line energy and equivalent width in quasar rest frame, units $\mathrm{eV}$. The corresponding mean wavelength value in $\AA$ is given within brackets.

${ }^{\mathrm{d}}$ Improvement in C-statistic upon adding line to model. 
Table 3. Soft X-ray Lines identified in 2011 XMM-Newton RGS.

\begin{tabular}{|c|c|c|c|}
\hline Line ID $^{a}$ & $\mathrm{E}_{\text {lab }}^{b}$ & $\mathrm{E}_{\text {quasar }}^{c}$ & $\mathrm{E}_{\mathrm{obs}}^{d}$ \\
\hline 1. N VI $1 s \rightarrow 2 p$ & 430.7 [28.787] & $430.3[28.813]$ & 405.1 [30.606] \\
\hline 2. $\mathrm{CVI} 1 s \rightarrow 3 p$ & 435.5 [28.469] & $435.6[28.463]$ & 409.8 [30.255] \\
\hline 3. $\mathrm{C}$ VI $1 s \rightarrow 4 p$ & 459.4 [26.988] & $459.0[27.012]$ & 432.3 [28.680] \\
\hline 4. $\mathrm{CVI} 1 s \rightarrow 5 p$ & $470.4[26.357]$ & $470.7[26.340]$ & 443.2 [27.975] \\
\hline 5. C VI $1 s \rightarrow 6 p$ & $476.4[26.025]$ & $476.0[26.047]$ & 448.1 [27.669] \\
\hline 6. C VI K-edge & 489.9 [25.308] & 489.7 [25.318] & 460.0 [26.953] \\
\hline 7. $\mathrm{N}$ VI $1 s \rightarrow 3 p$ & 496.7 [24.962] & $497.3[24.931]$ & 467.4 [26.526] \\
\hline 8. $\mathrm{N} \mathrm{VII}^{e} 2 p \rightarrow 1 s$ & $500.4[24.777]$ & $500.3[24.782]$ & 470.2 [26.368] \\
\hline 9. $\mathrm{N}$ VII $1 s \rightarrow 2 p$ & $500.4[24.777]$ & $501.1[24.742]$ & 470.9 [26.329] \\
\hline 10. N VI $1 s \rightarrow 4 p$ & $521.6[23.770]$ & $521.8[23.761]$ & 491.1 [25.246] \\
\hline 11. Ov $1 s \rightarrow 2 p$ & $554.5[22.360]$ & $554.2[22.372]$ & $521.8[23.761]$ \\
\hline 12. O VII ${ }^{e}$ & & 564 [21.983] & 530 [23.393] \\
\hline 13. O VI $1 s \rightarrow 2 p$ & $562.6[22.038]$ & 564.0 [21.983] & 530.1 [23.389] \\
\hline 14. O VI $1 s \rightarrow 2 p$ & $568.2[21.821]$ & $568.6[21.805]$ & $535.2[23.166]$ \\
\hline 15. N VII $1 s \rightarrow 3 p$ & $592.9[20.911]$ & $592.9[20.911]$ & 558.1 [22.215] \\
\hline 16. N VII $1 s \rightarrow 4 p$ & $625.4[19.825]$ & 625.0 [19.837] & 588.3 [21.075] \\
\hline 17. N VII $1 s \rightarrow 5 p$ & 640.4 [19.360] & $640.2[19.366]$ & $602.6[20.575]$ \\
\hline 18. O VIII $1 s \rightarrow 2 p$ & 653.5 [18.972] & 653.3 [18.978] & 614.9 [20.163] \\
\hline 19. $\mathrm{OVIII}^{e} 2 p \rightarrow 1 s$ & 653.5 [18.972] & 654.5 [18.943] & 615.1 [20.157] \\
\hline 20. O VII $1 s \rightarrow 3 p$ & $665.6[18.627]$ & 665.9 [18.619] & 626.8 [19.781] \\
\hline 21. O VII $1 s \rightarrow 4 p$ & $697.1[17.786]$ & $697.6[17.773]$ & $656.6[18.883]$ \\
\hline 22. OVII $1 s \rightarrow 5 p$ & 712.7 [17.396] & $712.8[17.394]$ & 670.9 [18.480] \\
\hline 23. O VII $1 s \rightarrow 6 p$ & 720.7 [17.203] & 721.2 [17.191] & 678.8 [18.265] \\
\hline \multicolumn{4}{|l|}{ 24. Fe M UTA } \\
\hline 25. O VIII $1 s \rightarrow 3 p$ & $774.6[16.006]$ & $774.4[16.010]$ & 728.9 [17.010] \\
\hline 26. Fe XVII & & $812.4[15.261]$ & 764.7 [16.213] \\
\hline 27. O VIII $1 s \rightarrow 4 p$ & 816.9 [15.177] & 816.4 [15.187] & 768.4 [16.135] \\
\hline 28. Fe XVII-XIX & & $825.9[15.012]$ & 777.4 [15.949] \\
\hline 29. O VIII $1 s \rightarrow 5 p$ & $836.6[14.820]$ & 836.2 [14.827] & 787.1 [15.752] \\
\hline 30. O VIII $1 s \rightarrow 6 p$ & 847.2 [14.635] & 846.8 [14.642] & 797.0 [15.556] \\
\hline 31. $\mathrm{Ne} \mathrm{V}^{i}$ & $873.7[14.191]$ & 874.2 [14.183] & 821.7 [15.085] \\
\hline 32. Ne VII ${ }^{i}$ & $898.8[13.794]$ & 898.1 [13.805] & 844.1 [14.688] \\
\hline 33. $\mathrm{Ne} \mathrm{IX}^{e}$ & & $922.1[13.446]$ & 866.6 [14.307] \\
\hline 34. Ne VIII ${ }^{i}$ & 909.2 [13.637] & $909.4[13.634]$ & 854.7 [14.506] \\
\hline 35. Fe XIX & & 916.8 [13.524] & 862.9 [14.368] \\
\hline 36. Ne IX $1 s \rightarrow 2 p$ & 922.0 [13.447] & 921.9 [13.449] & 867.7 [14.289] \\
\hline 37. Fe $\mathrm{xx}$ & & $965.5[12.841]$ & 908.9 [13.641] \\
\hline 38. NeX $1 s \rightarrow 2 p$ & 1021.5 [12.137] & 1021.5 [12.137] & 961.5 [12.895] \\
\hline
\end{tabular}


Table 3-Continued

\begin{tabular}{lccr}
\hline \hline \multicolumn{1}{c}{ Line ID $^{a}$} & $\mathrm{E}_{\text {lab }}^{b}$ & $\mathrm{E}_{\text {quasar }}^{c}$ & $\mathrm{E}_{\text {obs }}^{d}$ \\
\hline 39. $\mathrm{Mg} \mathrm{VIII}^{i}$ & $1306.4[9.491]$ & $1306.4[9.491]$ & $1227.9[10.097]$ \\
40. Mg IX & $1323.1[9.371]$ & $1322.5[9.375]$ & $1243.0[9.975]$ \\
41. Mg XI $1 s \rightarrow 2 p$ & $1353.3[9.162]$ & $1352.4[9.169]$ & $1272.9[9.687]$ \\
42. Mg XII $1 s \rightarrow 2 p$ & $1472.3[8.421]$ & $1471.9[8.423]$ & $1385.4[8.949]$ \\
\hline
\end{tabular}

${ }^{a}$ Line identification. Line number corresponds to those marked Figure 5 and Figure 6 .

${ }^{\mathrm{b}}$ Known atomic/lab frame energy of line in units eV. The corresponding wavelength in $\AA$ is given within brackets. Values are from www.nist.gov

${ }^{\mathrm{c}}$ Measured line energy in the quasar rest frame in $\mathrm{eV}$. The corresponding wavelength in $\AA$ is given within brackets.

${ }^{\mathrm{d}}$ Measured line energy in the observed frame in eV. Typical uncertainty is within $\pm 1 \mathrm{eV}$. The corresponding wavelength in $\AA$ is given within brackets.

${ }^{\mathrm{e}}$ Possible emission line.

${ }^{i}$ Possible inner-shell absorption line. Known atomic energy taken from Behar \& Netzer (2002). 
Table 4. Gaussian Fits to Velocity Profiles of $\mathrm{H}$ and He-like Absorption Lines

\begin{tabular}{|c|c|c|c|c|c|}
\hline Line & Instrument & $\sigma_{\mathrm{obs}}{ }^{a}$ & $\sigma_{\text {int }}^{b}$ & $v_{\text {out }}^{c}$ & $\Delta \chi^{2 d}$ \\
\hline \multicolumn{6}{|l|}{ H-like:- } \\
\hline C VI Ly- $\beta$ (a) & RGS & $320 \pm 80$ & $<270$ & $-444 \pm 73$ & 43.0 \\
\hline C VI Ly- $\beta(\mathrm{b})^{e}$ & RGS & - & - & $-1840 \pm 100^{e}$ & 16.0 \\
\hline N VII Ly- $\alpha$ (a) & RGS & $480 \pm 95$ & $360_{-140}^{+120}$ & $-450 \pm 94$ & 57.5 \\
\hline $\mathrm{N}$ VII Ly- $\alpha(\mathrm{b})^{e}$ & RGS & - & - & $-2020 \pm 120^{e}$ & 8.2 \\
\hline O VIII Ly- $\alpha$ & RGS & $297 \pm 65$ & $<200$ & $-353 \pm 62$ & 47.0 \\
\hline Ne X Ly- $\alpha$ & HEG & $415 \pm 135$ & $395_{-145}^{+170}$ & $-227 \pm 123$ & 33.1 \\
\hline Mg XII Ly- $\alpha$ & HEG & $<120$ & - & $<40$ & 31.2 \\
\hline Si XIV Ly- $\alpha$ & HEG & $<125$ & - & $<340$ & 8.7 \\
\hline \multicolumn{6}{|l|}{ He-like:- } \\
\hline N VI He- $\alpha$ (a) & RGS & $320 \pm 90$ & $<280$ & $-428 \pm 88$ & 26.2 \\
\hline $\mathrm{N}$ VI He- $\alpha(\mathrm{b})^{e}$ & RGS & - & - & $-1990 \pm 120^{e}$ & 12.1 \\
\hline $\mathrm{O}$ VII He- $\beta$ & RGS & $600 \pm 180$ & $460_{-280}^{+220}$ & $-900 \pm 180$ & 22.3 \\
\hline $\mathrm{Ne}$ IX He- $\beta$ & HEG & $440 \pm 190$ & $420_{-200}^{+180}$ & $<234$ & 14.1 \\
\hline $\mathrm{Mg}$ XI He- $\alpha$ & HEG & $310 \pm 175$ & $<455$ & $<170$ & 7.0 \\
\hline Si XIII He- $\alpha$ & HEG & $<120$ & - & $<163$ & 8.8 \\
\hline
\end{tabular}

${ }^{\text {a }}$ Observed $1 \sigma$ width of absorption line in $\mathrm{km} \mathrm{s}^{-1}$.

${ }^{\mathrm{a}}$ Intrinsic $1 \sigma$ width of absorption line in $\mathrm{km} \mathrm{s}^{-1}$ after correcting for instrument spectral resolution.

${ }^{c}$ Velocity shift of absorption line in $\mathrm{km} \mathrm{s}^{-1}$. Negative values donate blue-shift.

${ }^{\mathrm{d}}$ Improvement in fit statistic after modeling Gaussian absorption profile.

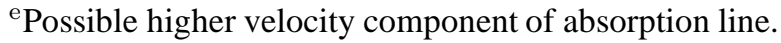


Table 5. Warm Absorber Parameters from RGS and HETG Spectra

\begin{tabular}{|c|c|c|c|c|}
\hline Component & Parameter & RGS 2011 & HETG 2011 & HETG 2002 \\
\hline Power-Law & $\Gamma$ & $2.32 \pm 0.08$ & $2.13_{-0.10}^{+0.11}$ & $=2011^{t}$ \\
\hline (uncovered) & $f_{\text {uncov }}{ }^{a}$ & $0.39_{-0.02}^{+0.03}$ & $0.23 \pm 0.03$ & $0.18 \pm 0.02$ \\
\hline Warm Absorber & $N_{\mathrm{H}}^{b}$ & $2.12 \pm 0.07$ & $2.10_{-0.23}^{+0.19}$ & $1.43_{-0.37}^{+0.34}$ \\
\hline \multirow[t]{2}{*}{ (Component 1) } & $\log \left(\xi / \text { erg cm s}{ }^{-1}\right)^{c}$ & $1.27 \pm 0.02$ & $1.15 \pm 0.05$ & $0.91 \pm 0.16$ \\
\hline & $v_{\text {out }}{ }^{d}$ & $-480 \pm 40$ & $-315 \pm 40$ & $-290 \pm 150$ \\
\hline$\Delta C$ or $\Delta \chi^{2 e}$ & - & 2065 & 176.2 & - \\
\hline Warm Absorber & $N_{\mathrm{H}}^{b}$ & $1.50 \pm 0.20$ & $1.5_{-0.5}^{+0.3}$ & $1.2_{-0.7}^{+0.9}$ \\
\hline \multirow[t]{2}{*}{ (Component 2) } & $\log \left(\xi / \operatorname{erg}_{\mathrm{cms}}{ }^{-1}\right)^{c}$ & $2.04_{-0.07}^{+0.04}$ & $2.14_{-0.11}^{+0.10}$ & $2.03_{-0.13}^{+0.23}$ \\
\hline & $v_{\text {out }}{ }^{d}$ & $-470 \pm 60$ & $-260_{-60}^{+30}$ & $-150_{-140}^{+130}$ \\
\hline$\Delta C$ or $\Delta \chi^{2 e}$ & - & 244.6 & 148.1 & - \\
\hline Warm Absorber & $N_{\mathrm{H}}^{b}$ & $3.6 \pm 1.3$ & $1.7_{-0.6}^{+0.7}$ & $2.3_{-1.4}^{+2.5}$ \\
\hline \multirow[t]{2}{*}{ (Component 3) } & $\log \left(\xi / \text { erg cms s}{ }^{-1}\right)^{c}$ & $2.80_{-0.07}^{+0.05}$ & $2.88_{-0.14}^{+0.11}$ & $2.9_{-0.3}^{+0.4}$ \\
\hline & $v_{\text {out }}{ }^{d}$ & $<130$ & $<70$ & $-380_{-220}^{+200}$ \\
\hline$\Delta C$ or $\Delta \chi^{2 e}$ & - & 18.5 & 33.7 & - \\
\hline Partial Coverer & $N_{\mathrm{H}}^{b}$ & $60.0^{f}$ & $55_{-3}^{+2}$ & $=2011^{t}$ \\
\hline \multirow[t]{2}{*}{$(\mathrm{pc} 1)$} & $\log \left(\xi / \operatorname{erg} \mathrm{cm} \mathrm{s}^{-1}\right)^{c}$ & $1.0^{f}$ & $1.04_{-0.11}^{+0.08}$ & $=2011^{t}$ \\
\hline & $f_{\mathrm{cov} 1}^{g}$ & $0.61 \pm 0.05$ & $0.40 \pm 0.10$ & $0.39 \pm 0.07$ \\
\hline$\Delta C$ or $\Delta \chi^{2 e}$ & - & 213.1 & 193.1 & - \\
\hline Partial Coverer & $N_{\mathrm{H}}^{b}$ & - & $690_{-100}^{+90}$ & $=2011^{t}$ \\
\hline \multirow[t]{2}{*}{$(\mathrm{pc} 2)$} & $\log \left(\xi / \text { erg cm s}{ }^{-1}\right)^{c}$ & - & $1.04^{f}$ & $=2011^{t}$ \\
\hline & $f_{\operatorname{cov} 2^{g}}$ & - & $0.37 \pm 0.10$ & $0.43 \pm 0.11$ \\
\hline$\Delta C$ or $\Delta \chi^{2 e}$ & - & - & 31.6 & - \\
\hline \multirow[t]{2}{*}{ Total Flux ${ }^{h}$} & $F_{0.5-2.0}$ & $1.80 \pm 0.01$ & $1.33 \pm 0.01$ & $0.75 \pm 0.01$ \\
\hline & $F_{2.0-10.0}$ & - & $3.8 \pm 0.1$ & $2.5 \pm 0.1$ \\
\hline
\end{tabular}

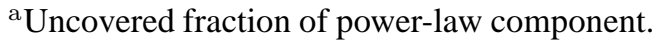

${ }^{\mathrm{b}}$ Hydrogen column density, units $\times 10^{21} \mathrm{~cm}^{-2}$.

${ }^{\mathrm{c}} \log$ ionization parameter.

${ }^{\mathrm{d}}$ Outflow velocity in units $\mathrm{km} \mathrm{s}^{-1}$. Negative values indicate outflow.

${ }^{\text {e }}$ Improvement in either C-statistic (HETG) or $\chi^{2}$ (RGS) upon the addition of the component to the model.

${ }_{\mathrm{f}}^{\mathrm{f}}$ Indicates parameter is fixed.

${ }^{\mathrm{g}}$ Covering fraction of partial covering component

${ }^{\mathrm{h}} 0.5-2.0 \mathrm{keV}$ or $2-10 \mathrm{keV}$ band flux, units $\times 10^{-11} \mathrm{erg} \mathrm{cm}^{-2} \mathrm{~s}^{-1}$. 
${ }^{t}$ Parameter is tied to the 2011 HETG value. 
Table 6. Soft X-ray Emission Lines in 2011 RGS and Chandra HETG Spectra

\begin{tabular}{lcccccc}
\hline \hline \multicolumn{1}{c}{ Line ID } & \multicolumn{1}{c}{$\mathrm{E}_{\text {quasar }}^{a}$} & Flux $^{b}$ & $\mathrm{EW}^{c}$ & $\sigma_{\mathrm{v}}{ }^{d}$ & FWHM $^{e}$ & $\Delta \chi^{2}$ or $\Delta C^{g}$ \\
\hline RGS:- & & & & & & \\
C VI Ly- $\alpha$ & $363_{-3.5}^{+2.5}[34.155]$ & $34_{-11}^{+14}$ & $2.5_{-0.8}^{+1.0}$ & $4400_{-600}^{+500 t}$ & $10200_{-1400}^{+1200 t}$ & 43.9 \\
N VI & $419.3_{-2.0}^{+1.5}[29.569]$ & $9.2_{-3.2}^{+2.6}$ & $1.1 \pm 0.4$ & $1800_{-600}^{+900}$ & $4200_{-1400}^{+2100}$ & 16.6 \\
N VII Ly- $\alpha$ & $498.7 \pm 0.2[24.861]$ & $11.3_{-1.8}^{+4.5}$ & $0.9_{-0.2}^{+0.4}$ & $340 \pm 130$ & $780 \pm 300$ & 75.1 \\
O VII (broad) & $564.5 \pm 0.9[21.964]$ & $38.3_{-4.9}^{+4.2}$ & $8.3_{-1.1}^{+0.9}$ & $4400_{-600}^{+500}$ & $10200_{-1400}^{+1200}$ & 345.1 \\
O VII (narrow) & $561 \pm 1[22.100]$ & $4.8 \pm 2.0$ & $0.9 \pm 0.4$ & $<530$ & $<1250$ & 16.0 \\
O VIII Ly- $\alpha$ & $654.5 \pm 1.0[18.943]$ & $6.4 \pm 1.5$ & $1.7 \pm 0.4$ & $1650_{-700}^{+1400}$ & $3900_{-1600}^{+3300}$ & 28.8 \\
Ne IX & $905.1 \pm 1.3[13.698]$ & $1.2 \pm 0.5$ & $0.9 \pm 0.4$ & $<1260$ & $<2700$ & 9.7 \\
\hline O VII line $:^{h}$ & & & & & & \\
O VII (f) & $561.0^{f}[22.100]$ & $26 \pm 4$ & $5.1 \pm 0.8$ & $3160_{-600}^{+400}$ & $7300_{-1500}^{+1000}$ & - \\
O VII (i) & $568.6^{f}[21.805]$ & $9 \pm 6$ & $1.7 \pm 1.1$ & - & - & - \\
O VII (r) & $573.9^{f}[21.604]$ & $<9$ & $<1.7$ & - & - & - \\
\hline HETG:- & & & & & & \\
O VII & $567 \pm 5[21.867]$ & $20_{-9}^{+11}$ & $7.4_{-3.3}^{+4.1}$ & $4300_{1600}^{+2000}$ & $9900_{-3700}^{+4600}$ & 17.0 \\
Ne IX (narrow) & $905.3 \pm 0.9[13.695]$ & $0.9_{-0.5}^{+0.6}$ & $1.0 \pm 0.6$ & $<600$ & $<1400$ & 10.6 \\
Ne IX (broad) & $940_{-30}^{+6}[13.190]$ & $2.6_{-1.6}^{+3.2}$ & $3.0_{-1.8}^{+3.7}$ & $2100_{-1300}^{+1900}$ & $4800_{-3000}^{+4400}$ & 9.8 \\
\hline
\end{tabular}

${ }^{a}$ Measured line energy in quasar rest frame, units $\mathrm{eV}$. The corresponding mean wavelength value in $\AA$ is given within brackets.

${ }^{\mathrm{b}}$ Line photon flux, units $\times 10^{-5}$ photons $\mathrm{cm}^{-2} \mathrm{~s}^{-1}$

${ }^{\mathrm{c}}$ Equivalent width in quasar rest frame, units $\mathrm{eV}$.

${ }^{\mathrm{d}} 1 \sigma$ velocity width, units $\mathrm{km} \mathrm{s}^{-1}$.

${ }^{\mathrm{e}} \mathrm{FWHM}$ velocity width, units $\mathrm{km} \mathrm{s}^{-1}$.

${ }_{\mathrm{f}}^{\mathrm{f}}$ Indicates parameter is fixed.

${ }^{\mathrm{g}}$ Improvement in C-statistic or $\Delta \chi^{2}$ upon adding line to model.

${ }^{\mathrm{h}} \mathrm{RGS}$ deconvolution of broad $\mathrm{O}$ VII line into forbidden, intercombination and resonance line components.

${ }^{\mathrm{t}}$ Line velocity width of $\mathrm{C}$ VI line tied to broad O VII line. 
Table 7. Properties of the fully covered warm absorber components ("components 1,2, and 3") and the highly ionized component ("component high") discussed in $\$ 4.4$ The values of $N_{\mathrm{H}}, \log \xi$ and $v_{\text {out }}$ are those inferred from the 2011 RGS observations (see Table 5) for components 1, 2, and 3, and from the

HETG observations for the highly ionized absorber. See text for full definitions of the parameters.

\begin{tabular}{lcccc}
\hline \hline \multicolumn{1}{c}{ Parameters } & component 1 & component 2 & component 3 & component high \\
\hline$N_{\mathrm{H}}\left(\times 10^{21} \mathrm{~cm}^{-2}\right)$ & $2.12 \pm 0.07$ & $1.50 \pm 0.20$ & $3.6 \pm 1.3$ & $>150$ \\
$\log \left(\xi / \mathrm{erg} \mathrm{cm} \mathrm{s}^{-1}\right)$ & $1.27 \pm 0.02$ & $2.04_{-0.07}^{+0.04}$ & $2.80_{-0.07}^{+0.05}$ & $4.8_{-0.8}^{+1.0}$ \\
$v_{\text {out }}{ }^{d}\left(\mathrm{~km} \mathrm{~s}^{-1}\right)$ & $-480 \pm 40$ & $-470 \pm 60$ & $<130$ & $-15600 \pm 2400$ \\
$r_{\min }(\mathrm{cm}) /(\mathrm{pc})$ & $2.8 \times 10^{19} / 9.0$ & $2.9 \times 10^{19} / 9.4$ & $3.8 \times 10^{20} / 122$ & $2.6 \times 10^{16} / 0.008$ \\
$r_{\max }{ }^{a}(\mathrm{~cm}) /(\mathrm{pc})$ & $5.3 \times 10^{19} / 17.2$ & $1.2 \times 10^{22} / 3940$ & $8.8 \times 10^{20} / 285$ & $2.1 \times 10^{17} / 0.068$ \\
$\dot{M}_{\text {out }}\left(\times 10^{25} \mathrm{~g} / \mathrm{s}\right)$ & {$[1.9-3.6]$} & {$[1.4-560]$} & {$[11.8-27.3]$} & $>4.0$ \\
$\dot{M}_{\text {out }}(\dot{M} / \mathrm{yr})$ & {$[0.3-0.6]$} & {$[0.2-89]$} & {$[1.9-4.3]$} & $>0.6$ \\
$\dot{E}_{\mathrm{K}} / L_{\mathrm{bol}} b(\%)$ & {$\left[5.1 \times 10^{-4}-9.8 \times 10^{-4}\right]$} & {$\left[3.5 \times 10^{-4}-0.14\right]$} & {$\left[2.4 \times 10^{-4}-5.4 \times 10^{-4}\right]$} & $>1.1$ \\
$\dot{P}_{\text {out }} / \dot{P}_{\text {rad }}(\%)$ & {$[0.63-1.2]$} & {$[0.45-184]$} & {$[1.1-2.5]$} & $>46$ \\
\hline \hline
\end{tabular}

${ }^{\mathrm{a}} r_{\max }$ is inferred from eq. 6.2.3, except for component 1 for which $r_{\max }$ corresponds to $r_{\text {var }}$ inferred from recombination time-scale, see 86.2 .2 .

${ }^{\mathrm{b}} L_{\mathrm{bol}}=4.3 \times 10^{45} \mathrm{erg} \mathrm{s}^{-1}$ (Dunn et al. 2008), and $L_{\mathrm{Edd}}=3.0 \times 10^{46} \mathrm{erg} \mathrm{s}^{-1}$. 